Aus der Klinik für Allgemein-,Viszeral- und Kinderchirurgie

(Prof. Dr. med. M. Ghadimi)

der Medizinischen Fakultät der Universität Göttingen

\title{
Biografische Besonderheiten und Persönlichkeitsmerkmale habilitierter Chirurginnen und Chirurgen
}

\author{
INAUGURAL-DISSERTATION \\ zur Erlangung des Doktorgrades \\ der Medizinischen Fakultät der \\ Georg-August-Universität zu Göttingen
}

\author{
vorgelegt von \\ Laura Sophie Schwarz
}

aus Kappeln

Göttingen 2018 
Dekan:

Referent/in

Ko-Referent/in:

Drittreferent/in:
Prof. Dr. rer. nat. H. K. Kroemer

Prof. Dr. med. S. König (Universitätsklinikum Würzburg)

Datum der mündlichen Prüfung: 
Hiermit erkläre ich, die Dissertation mit dem Titel "Biografische Besonderheiten und Persönlichkeitsmerkmale habilitierter Chirurginnen und Chirurgen" eigenständig angefertigt und keine anderen als die von mir angegebenen Quellen und Hilfsmittel verwendet zu haben.

Göttingen, den 


\section{Inhaltsverzeichnis}

Abbildungsverzeichnis .................................................................... II

Tabellenverzeichnis ....................................................................... IV

Abkürzungsverzeichnis ................................................................. V

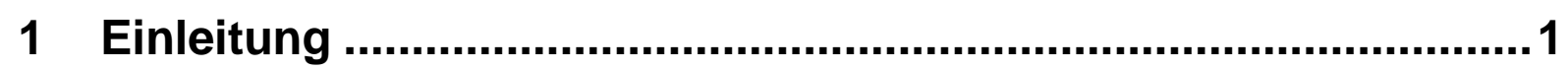

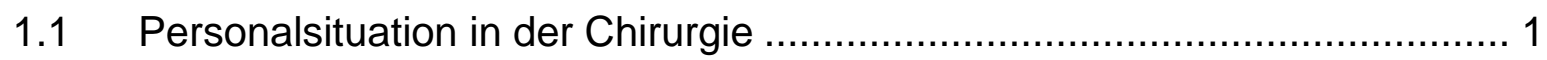

1.2 Geschlechterverteilung in medizinischer Aus- und Weiterbildungszeit......... 2

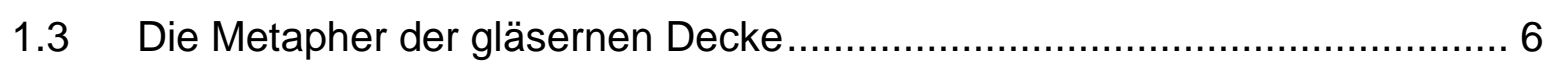

1.4 Habilitation als Qualifizierungsmerkmal............................................... 6

$1.5 \quad$ Fragestellung und Ziel..................................................................... 7

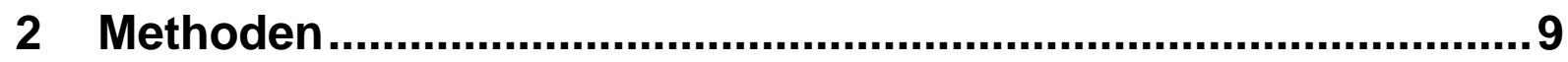

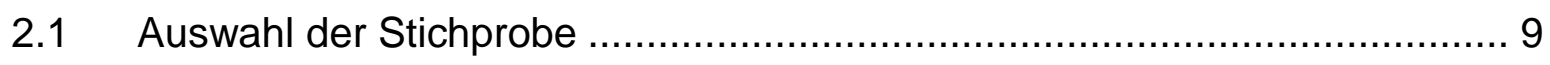

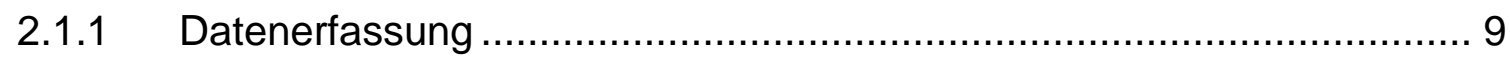

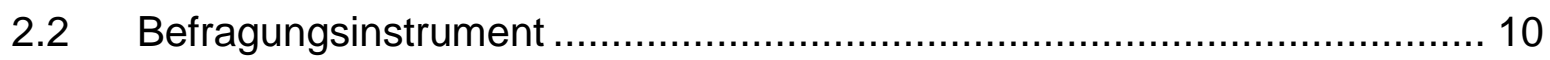

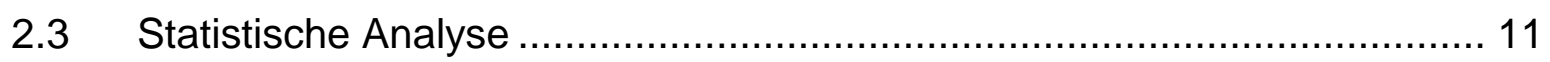

3 Ergebnisse .......................................................................... 13

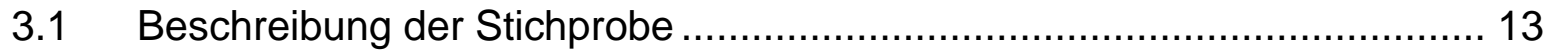

3.2 Geschlechtsabhängiger Einfluss auf eine chirurgische Karriere …........... 18

3.2.1 Bewertung der Motive für die Wahl der Weiterbildung in der Chirurgie 18

3.2.2 Bewertung von Karrierefaktoren .................................................. 20

3.2.3 Bewertung von Persönlichkeitsmerkmalen ...................................... 22

3.3 Geschlechtsunabhängiger Einfluss auf eine chirurgische Karriere ........... 25

3.3.1 Bewertung karrierebeeinflussender Maßnahmen im Arbeitsumfeld .... 25

3.4 Zufriedenheit der Teilnehmenden ..................................................... 29

3.4.1 Signifikante Unterschiede zwischen besonders zufriedenen und

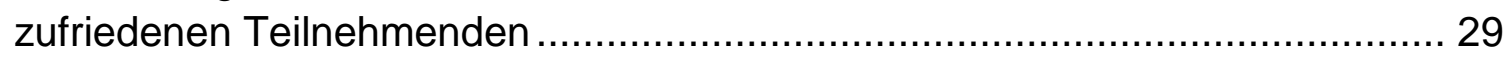

3.4.2 Prädiktoren für eine hohe berufliche Zufriedenheit ............................ 31

3.5 Einfluss von Elternschaft auf eine chirurgische Karriere .......................... 33

3.5.1 Signifikante Unterschiede zwischen kinderlosen Teilnehmenden und Teilnehmenden mit Kind..... 33 


\section{Diskussion}

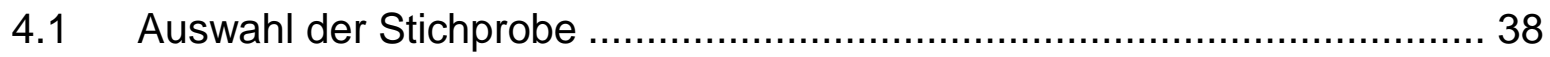

$4.2 \quad$ Wahl der Weiterbildungsdisziplin Chirurgie .......................................... 39

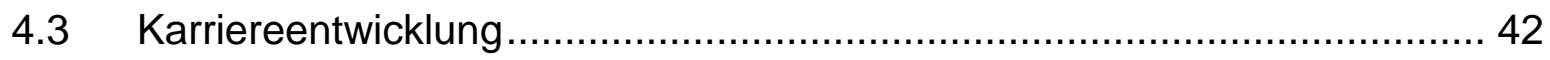

4.3.1 Persönlichkeitsstruktur in der Chirurgie .................................... 42

4.3.2 Externe Karrierefaktoren ..................................................... 45

4.3.3 Einfluss von Elternschaft, Familie und Schwangerschaft auf die Karriereentwicklung ........................................................................ 46

4.4 Work-Life-Integration und Zufriedenheit in akademischer Chirurgie.......... 50

4.5 Notwendigkeit karrierebeeinflussender Maßnahmen im Arbeitsumfeld ...... 52

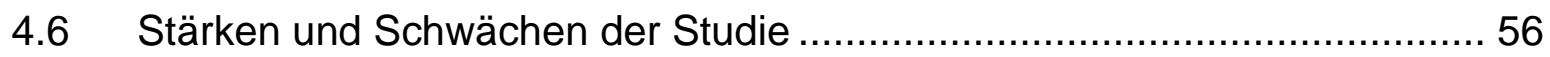

5 Zusammenfassung ...............................................................58

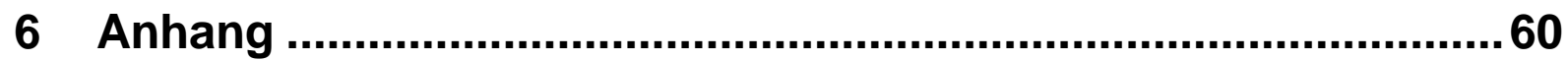

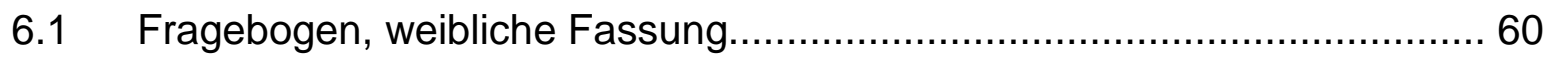

6.2 Zusätzliche berufliche Qualifikationen der Teilnehmenden ...................... 71

6.3 Berufliche Tätigkeiten der Partnerinnen und Partner der Teilnehmenden.. 73

7 Literaturverzeichnis ............................................................. 75

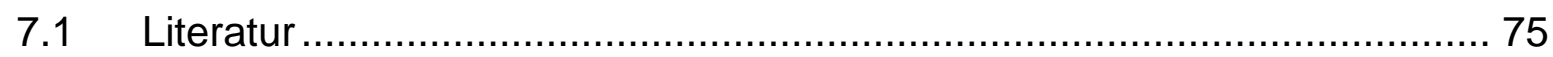

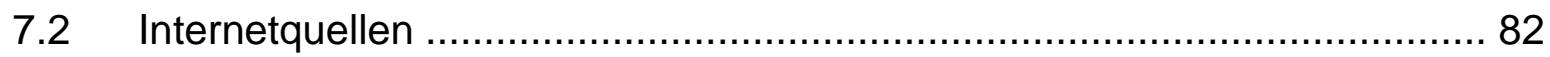




\section{Abbildungsverzeichnis}

Abbildung 1: Prozentuale Darstellung der Geschlechterverteilung unter deutschen

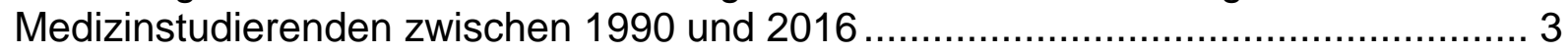

Abbildung 2: Prozentuale Darstellung der Geschlechterverteilung auf

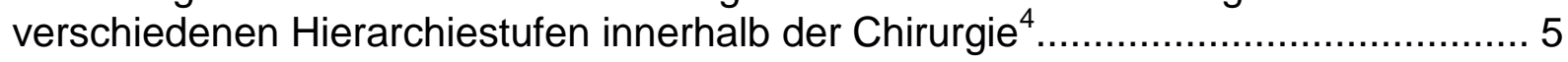

Abbildung 3: $\quad$ Motive für die Wahl zur Weiterbildungsdisziplin Chirurgie .............. 19

Abbildung 4: Karrierefaktoren für die Karriereentwicklung ........................... 20

Abbildung 5: Förderliche Eigenschaften für die Karriereentwicklung .................. 22 


\section{Tabellenverzeichnis}

Tabelle 1: Deskriptive Daten ......................................................................... 13

Tabelle 2: Freitextantworten zu Karrierefaktoren ............................................... 21

Tabelle 3: Freitextantworten zu geschlechtsspezifischen erfolgsrelevanten

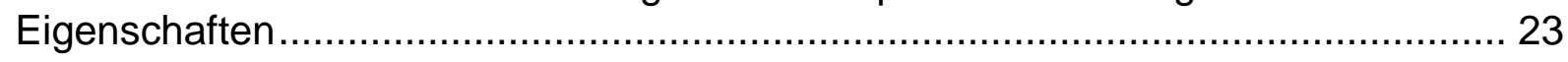

Tabelle 4: Karrierebeeinflussende Maßnahmen im Arbeitsumfeld ....................... 25

Tabelle 5: Freitextantworten zu wünschenswerten Veränderungen im

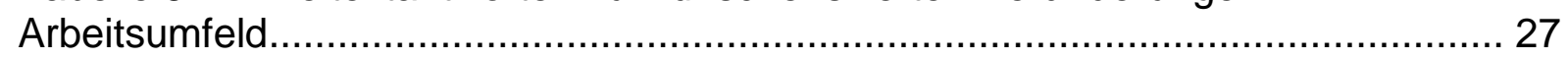

Tabelle 6: Unterschiede zwischen den beruflich besonders zufriedenen

Teilnehmenden und den beruflich zufriedenen Teilnehmenden .............................. 29

Tabelle 7: Prädiktoren einer hohen beruflichen Zufriedenheit ............................. 32

Tabelle 8: Signifikante Unterschiede zwischen kinderlosen Teilnehmenden und Teilnehmenden mit Kind ................................................................................... 34

Tabelle 9: $\quad$ Signifikante Unterschiede zwischen Müttern und Vätern ..................... 35 


\section{Abkürzungsverzeichnis}

\begin{tabular}{|l|l|}
\hline FFM & Fünf-Faktoren-Model \\
\hline FS & Fachsemester \\
\hline KarMed & $\begin{array}{l}\text { Studie zu Karriereverläufen und Karrierebrüchen bei } \\
\text { Ärztinnen und Ärzten während der fachärztlichen } \\
\text { Weiterbildung der Universität Hamburg }\end{array}$ \\
\hline M & Mittelwert \\
\hline PJ & Praktisches Jahr \\
\hline SD & Standardabweichung \\
\hline WS & Wintersemester \\
\hline
\end{tabular}




\section{$1 \quad$ Einleitung}

\subsection{Personalsituation in der Chirurgie}

Der sich abzeichnende chirurgische Nachwuchsmangel wird auf Grundlage aktueller Prognosen zunehmend diskutiert (Albrecht 2010; Scheuerlein und Settmacher 2010; Weise 2010). Schätzungen zufolge schlagen etwa $5 \%$ aller Absolventen und Absolventinnen eines Jahrgangs eine chirurgische Laufbahn ein, bei gleichbleibendem Bedarf müsste jedoch die doppelte Anzahl - über 10\% - aller angehenden Ärztinnen und Ärzte diesen Weg wählen, um dem aktuellen chirurgischen Versorgungsniveau gerecht zu werden (Berufsverband deutscher Chirurgen e.V. - Nachwuchsmangel und Nachwuchsförderung in der Chirurgie 2014). Der chirurgische Bedarf kann somit bei ausbleibendem Nachwuchs nicht sichergestellt werden (Williams Jr. und Ellison 2008). Dieses liegt zum einen am steigenden Anteil älterer multimorbider Menschen, zum anderen wird der Ärztemangel durch Abwanderung ins Ausland und Ruhestand älterer Mediziner verschärft (Bundesärztekammer und Kassenärztliche Bundesvereinigung - Dem deutschen Gesundheitswesen gehen die Ärzte aus! Studie zur Altersstruktur und Arztzahlentwicklung 2010).

Während des Studiums hat ein Großteil der Studierenden Interesse an der Chirurgie, und sie äußern sogar den Wunsch nach einer expliziten Forschungszeit (Sutton et al. 2014). Die Affinität zur Chirurgie scheint jedoch während der medizinischen Ausbildung abzunehmen (Krüger 2009), was durch die Ergebnisse einer Umfrage unter PJ-Studierenden vom Thieme-Verlag gestützt werden kann. In dieser Umfrage schätzten die Teilnehmenden vor dem chirurgischen Tertial ihre Motivation für eine Weiterbildung in einer chirurgischen Disziplin relativ hoch mit 7,4 (von max. 10) Punkten ein, während der Wert nach dem chirurgischen Tertial auf 5,5 Punkte sank (Thieme.de - PJ-Umfrage 2014). Für viele Studierende, Ärztinnen und Ärzte stellt die Chirurgie keine Karriereoption dar, was dadurch begründet wird, dass die Arbeitsbelastung für sie nicht mit einem funktionierenden Privatleben und einer befriedigenden Work-Life-Balance vereinbar sei (Richards et al. 2009; Ginther et al. 2016). Neben mangelnder Partizipation in Führungsfragen, fehlender interpersoneller Kommunikation und unzureichender und unorganisierter Lehre und Weiterbildung 
(Gargiulo et al. 2006; Lowenstein et al. 2007; Kerr et al. 2016) stellt eine fehlende Vereinbarkeit von Privatleben und Beruf in der heutigen Zeit einen der signifikantesten Einflüsse für einen Ausstieg aus dem klinischen Alltag dar.

Neben der klinischen Versorgung ist die Diskrepanz zwischen Personalbedarf und Personalmangel in der akademischen Chirurgie ebenfalls so groß wie schon lange nicht mehr (Sheldon 2010). Die akademische Chirurgie umfasst die Komplexität der Patientenversorgung in einem operativen Fach und verlangt zusätzlich die Kombination von klinischer Versorgung und wissenschaftlicher Forschung (Wells 1996). Diese intellektuelle Herausforderung stellt den Anreiz und gleichzeitig die Hürde der Disziplin dar (Diener et al. 2014).

Die heutige allgemeine Patientenversorgung findet zunehmend in einem komplexen Spannungsfeld aus Handwerk, Wissenschaft, Lehre und Ökonomie statt. Neben der Forschungsarbeit und dem Aneignen sowie Durchführen von chirurgischen Eingriffen dürfen die Aspekte der Gesundheitsökonomie nicht in den Hintergrund geraten (Gittes 2006; Cobey 2010). Betriebsorganisation, Personalführung und der Umgang mit Kürzungen von finanziellen Mitteln gehören schon lange zum zusätzlichen Aufgabenfeld eines Mediziners (Menger und Laschke 2012). Letztlich ist zu wenig Zeit geblieben, um chirurgische Forschung von hoher Qualität durchzuführen (Menger et al. 2012).

Die akademische Chirurgie, die gewissermaßen eine Doppelkarriere bedeutet, wird durch die vorgenannten Spannungsfelder gefährdet und zunehmend unattraktiver für Chirurginnen und Chirurgen. Daher müssten Strukturänderungen geschaffen werden, um dieser Tendenz zu begegnen und die chirurgische akademische Laufbahn für den Nachwuchs attraktiver zu gestalten (Zerhouni 2005; Atesok et al. 2012; Gottesman 2013).

\subsection{Geschlechterverteilung in medizinischer Aus- und Weiterbildungszeit}

Der Vergleich der Geschlechterverteilung unter Medizinstudierenden des ersten Fachsemesters vom Wintersemester 1990/91 bis zum Wintersemester 2016/17 zeigt eine zunehmende Diskrepanz im Laufe der Jahre (siehe Abbildung 1). 


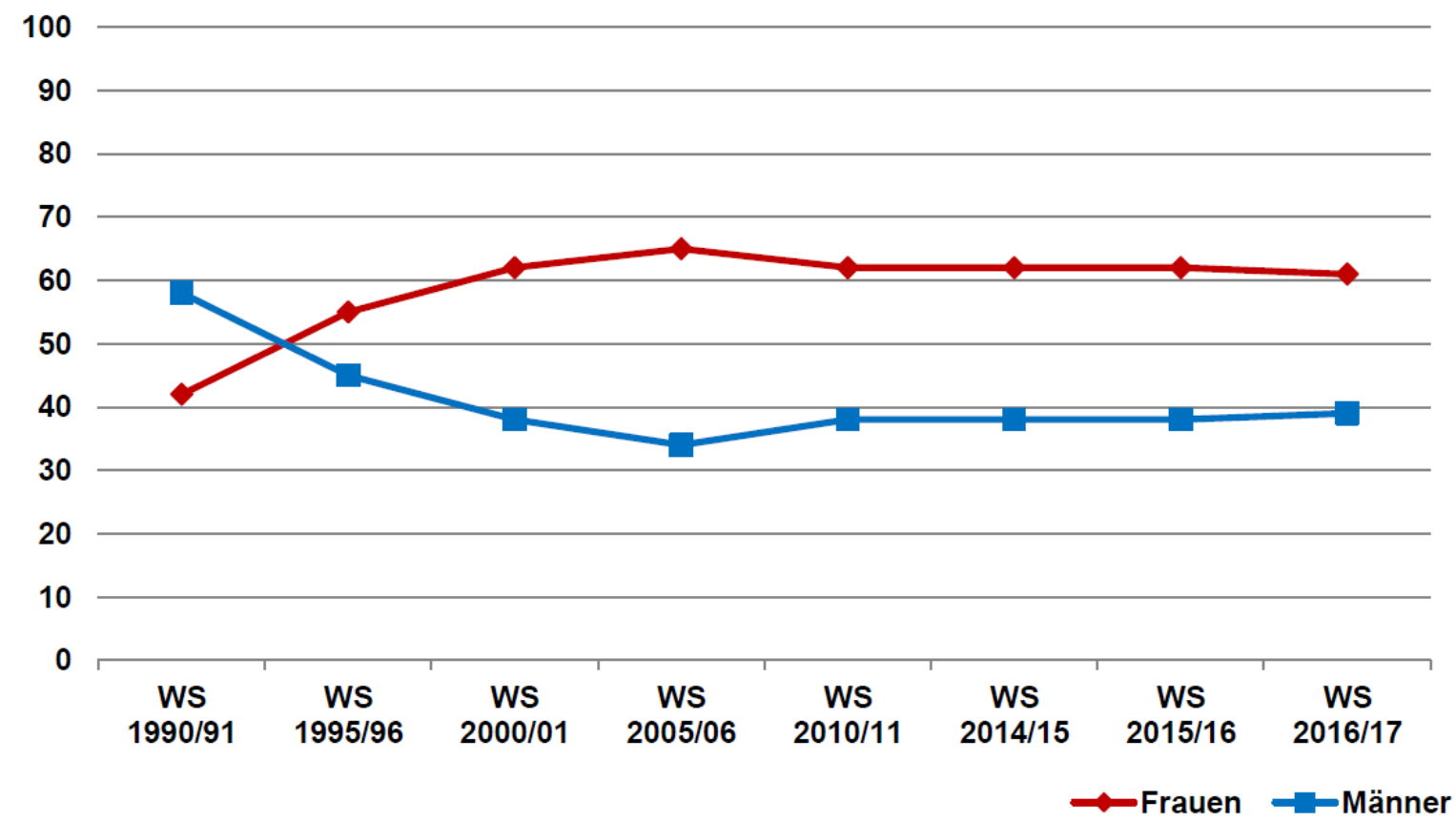

Abbildung 1: Prozentuale Darstellung der Geschlechterverteilung unter deutschen Medizinstudierenden zwischen 1990 und 2016

(Statistisches Bundesamt - Studierende an Hochschulen - Fachserie 11 Reihe 4.1 - Wintersemester 2017)

Der Anteil der Medizinstudentinnen stellte im Wintersemester 1990/91 mit 42 \% noch die Minderheit aller Medizinstudierenden dar, zehn Jahre später im Wintersemester 2000/01 lag dieser bereits bei $62 \%$. Der Trend hielt bis zum Wintersemester 2016/17 an (Statistisches Bundesamt - Studierende an Hochschulen - Fachserie 11 Reihe 4.1 - Wintersemester 2017). Im Prüfungsjahr 2016 absolvierten mehr Frauen (60\%) als Männer (40\%) ihr Staatsexamen in Humanmedizin, ebenso erlangte im selben Jahr ein größerer Anteil Frauen (59\%) als Männer (41\%) ihre Promotion in Humanmedizin (Statistisches Bundesamt - Prüfungen an Hochschulen - Fachserie 11 Reihe 4.2 2016).

Von allen in deutschen Krankenhäusern tätigen Ärztinnen und Ärzten mit abgeschlossener Weiterbildung befanden sich im Jahr 2016 12\% Frauen in einer leitenden Position. Eine Oberarztposition hatten 31\% Frauen inne, unter den Assistenzärztinnen und Assistenzärzten waren 56\% Frauen tätig (Statistisches Bundesamt- Grunddaten der Krankenhäuser- Fachserie 12 Reihe 6.1.1 2016). In der Literatur wird von einer „Feminisierung“ des Arztberufes gesprochen (Grözinger et al. 
2010). Betrachtet man den kumulierten Frauenanteil im weiteren Karriereverlauf, kann bei einer Umkehr der Zahlenverhältnisse von einer „Feminisierung“ allerdings keine Rede sein. Die Zahl der Habilitationen von Frauen im Fachbereich der Humanmedizin/Gesundheitswissenschaften stieg zwar kontinuierlich an, lag aber im Jahr 2016 mit 25,7\% insgesamt auf deutlich niedrigerem Niveau. (Statistisches Bundesamt - Pressemitteilungen - Frauenanteil bei den Habilitierten steigt weiterhin kontinuierlich an 2017). Gleichermaßen ist die Anzahl der Erst- oder Letztautorenschaften von Frauen in renommierten Fachzeitschriften in den letzten vier Jahren zwar stark gestiegen, aber auch hier ist der weibliche Autorinnenanteil noch wesentlich geringer als der männliche (Jagsi et al. 2006).

Sobald höhere Gehalts- und Hierarchiestufen erreicht werden, kehrt sich das Geschlechterverhältnis ins Gegenteil, sodass ein sogenannter Schereneffekt beim Voranschreiten der Karriere zu beobachten ist (Abele und Nitzsche 2002). Die Arbeit von Ziegler et al. bekrättigte diese Feststellung. Bei Ärztinnen in Weiterbildung bestünde wenig Interesse an einer Übernahme einer leitenden Position, ebenso absolvierten Ärztinnen seltener Ihre Weiterbildung auf universitärer Ebene, was Voraussetzung für eine akademische Laufbahn sei (Ziegler et al. 2017). Die Daten der Frauenanteile aus dem Fachgebiet der Chirurgie stützten diese Annahme. Abbildung 2 stellt die Divergenz zwischen den Geschlechtern auf den verschiedenen Hierarchiestufen innerhalb der chirurgischen Fachdisziplinen dar, der Schereneffekt nach der Ausbildung/Promotion und während der Weiterbildungszeit ist deutlich erkennbar. 


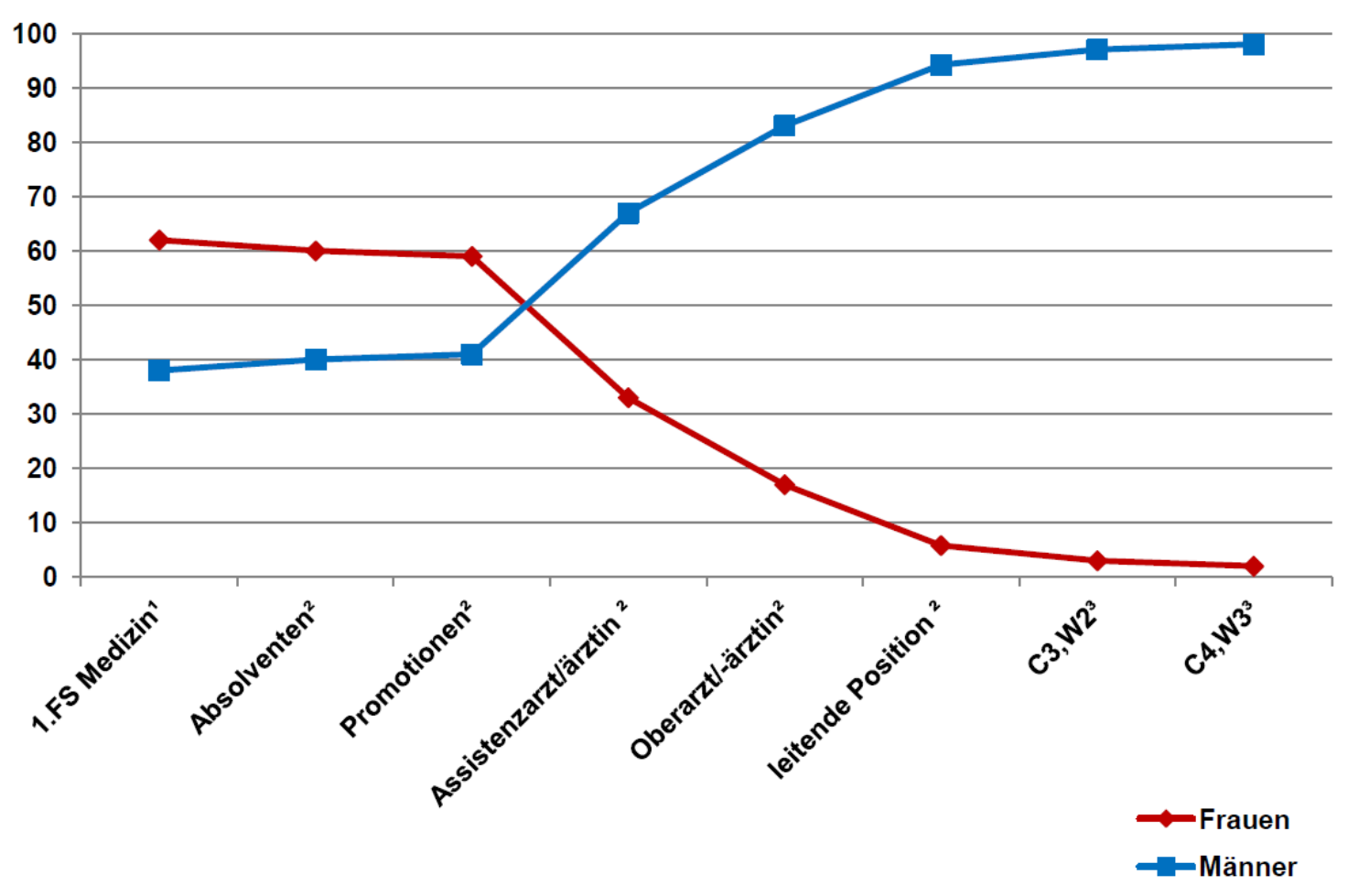

Abbildung 2: Prozentuale Darstellung der Geschlechterverteilung auf verschiedenen Hierarchiestufen innerhalb der Chirurgie ${ }^{4}$

${ }^{1}$ )WS 2016/2017: erstes Fachsemester 2)2016 ${ }^{3}$ )2015 ${ }^{4}$ )eingeschlossene Fachbereiche: Allgemeinchirurgie, Gefäßchirurgie, Herzchirurgie, Kinderchirurgie, plastische Chirurgie, Thoraxchirurgie, Unfallchirurgie, und Orthopädie, Viszeralchirurgie

(Statistisches Bundesamt - Fächergruppe Humanmedizin/Gesundheitswissenschaften, Lehr- und Forschungsbereich: Klinisch Praktische Humanmedizin (ohne Zahnmedizin) - Wissenschaftliches und künstlerisches Personal - Fachgebiet Chirurgie 2015; Statistisches Bundesamt - Prüfungen an Hochschulen Fachserie 11 Reihe 4.2 2016; Statistisches Bundesamt- Grunddaten der Krankenhäuser- Fachserie 12 Reihe 6.1.1 2016; Statistisches Bundesamt - Studierende an Hochschulen - Fachserie 11 Reihe 4.1 - Wintersemester 2017)

Während 2016 unter den chirurgischen Assistenzärztinnen und Assistenzärzten noch ein Anteil von 33\% Frauen tätig war, reduzierte sich die Zahl auf Ebene der Oberärztinnen und Oberärzten (17\%). Innerhalb der leitenden Positionen fanden sich in der Chirurgie lediglich noch 5,8\% Frauen (Statistisches Bundesamt- Grunddaten der Krankenhäuser- Fachserie 12 Reihe 6.1.1 2016).

Der Frauenanteil der an deutschen Hochschulen hauptberuflich tätigen Professorinnen und Professoren im Fachgebiet Chirurgie erreichte 2015 keine 5\%. Sowohl die C3/W2- Professur als auch die Stellen der C4/W3- Professur wurden von 
jeweils 3\% Frauen besetzt (Statistisches Bundesamt - Fächergruppe Humanmedizin/Gesundheitswissenschaften, Lehr- und Forschungsbereich: Klinisch Praktische Humanmedizin (ohne Zahnmedizin) - Wissenschaftliches und künstlerisches Personal - Fachgebiet Chirurgie 2015).

\subsection{Die Metapher der gläsernen Decke}

Nicht nur in Deutschland lässt sich das Missverhältnis zwischen Studienanfängerinnen und chirurgischen Assistenzärztinnen beobachten (Abele und Nitzsche 2002; Weber et al. 2005).

Die USA (Flannery 2002; Neumayer et al. 2002; Sexton et al. 2012; Cochran et al. 2013), Großbritannien (Richardson und Redfern 2000) und die Schweiz (Kaderli et al. 2011) sind ebenfalls mit diesem Problem konfrontiert. Der daraus resultierende Personalmangel in der Chirurgie (Weber et al. 2005) ließe sich auch nicht unbedingt durch eine zunehmende relative Anzahl von Frauen beheben, die eine chirurgische Laufbahn aufnehmen (Zhuge et al. 2011). Viele dieser Frauen stoßen zu einem späteren Zeitpunkt in ihrer beruflichen Laufbahn gegen die sogenannte "gläserne Decke“ (Mayr 2008). Die Metapher der gläsernen Decke steht für die Beobachtung, dass es noch immer ein Hindernis für Frauen gibt, in berufliche Führungsebenen einzutreten und in Berufspositionen aktiv zu sein, die traditionell eher zur Männerdomäne gezählt werden. Das Phänomen existiert keinesfalls nur im medizinischen Fachbereich (Abele-Brehm 2002; Lawler 2006), jedoch ist der Aufstieg für Frauen in der Medizin aus mittleren in höhere Positionen durch eine Vielzahl von Faktoren erschwert (Zhuge et al. 2011). Ein anderer Begriff, der diesen Zustand beschreibt, ist der des „Stickyfloors“ (Tesch et al. 1995). Dieser beinhaltet nicht nur die Tatsache, dass Frauen seltener eine Führungsposition erreichen, sondern auch, dass sie zu Beginn der Karriere weniger Unterstützung durch Arbeitgeber und Institution erfahren (Carnes et al. 2008).

\section{$1.4 \quad$ Habilitation als Qualifizierungsmerkmal}

Die Habilitation ist die bedeutsamste Hochschulprüfung in Deutschland. Sie dient dem Nachweis der besonderen Befähigung für Forschung und Lehre in einem bestimmten Fachgebiet und kann als wissenschaftliches Qualifizierungsmerkmal genutzt werden (Med.uni-Goettingen.de - Habilitationsordnung der Georg August Universität Göttingen 2009). Die Zulassung zur Habilitation setzt die Promotion und 
eine mehrjährige wissenschaftliche Tätigkeit voraus, die durch eine Habilitationsschrift oder durch vergleichbare Veröffentlichungen nachgewiesen wird. Nach Annahme der Habilitationsschrift durch die Habilitationskommission der Fakultät ist der Bewerbende zur Teilnahme an einem Kolloquium verpflichtet, während dem ein wissenschaftlicher Dialog zwischen dem Bewerbenden und der Habilitationskommission stattfindet. Nachdem eine mehrsemestrige Lehrtätigkeit durch den Bewerbenden nachgewiesen wurde, wird die Eignung einer Lehrbefugnis im Rahmen des Habilitationsverfahrens in einer Probevorlesung überprüft (Med.uniGoettingen.de - Habilitationsordnung der Georg August Universität Göttingen 2009). Mit der Habilitation wird der Habilitandin oder dem Habilitanden die Lehrbefugnis (Venia Legendi) an der Hochschule für ein Fachgebiet erteilt. Ab diesem Zeitpunkt darf dieser den Titel „Privatdozent/-in“ führen, was ihn berechtigt und verpflichtet, regelmäßig Lehrveranstaltungen abzuhalten. Privatdozentinnen und Privatdozenten können zu außerplanmäßigen Professorinnen und Professoren ernannt werden, sofern hervorragende Leistungen in Wissenschaft und Lehre an der Fakultät erbracht wurden (Med.uni-Goettingen.de - Habilitationsordnung der Georg August Universität Göttingen 2009).

\section{$1.5 \quad$ Fragestellung und Ziel}

Die zunehmende medizinische Nachfrage durch die Bevölkerung, der steigende Anteil an Medizinstudentinnen und das zunehmende Desinteresse an einer akademischen Karriere in der Chirurgie rufen langfristig ein Defizit im chirurgischen Nachwuchs hervor. Aus diesem Grund müssen die gegenwärtigen Umstände und Arbeitsverhältnisse wahrgenommen und überdacht werden.

Ziel der vorliegenden Arbeit war es, eine Bestandsaufnahme zur aktuellen Arbeitsund Lebenssituation der habilitierten Chirurginnen und Chirurgen in Deutschland durchzuführen. Unter der Annahme, dass bestimmte Charakteristika einen besonders förderlichen Einfluss auf eine chirurgische Karriere ausüben, wurde ein besonderer Fokus auf die persönlichen Merkmale der Teilnehmenden gelegt. Außerdem wurden externe Faktoren wie die strukturellen und organisatorischen Gegebenheiten erhoben (im Folgenden als Karrierefaktoren bezeichnet).

Auf dieser Informationsgrundlage könnten Verbesserungsvorschläge abgeleitet werden, um positive Aspekte zu fördern und negative Komponenten langfristig zu 
eliminieren. Ein Vergleich zwischen Frauen und Männern sollte helfen die Geschlechterdisparität in diesem Fachgebiet näher zu beleuchten, sodass auf der Basis des „Ist-Zustandes“ entsprechende Vorschläge zu Erneuerungen gemacht werden können. Die zentrale Frage in dieser Arbeit lautete, ob intrinsische oder extrinsische Merkmale existieren bzw. als solche wahrgenommen werden, die eine Karriere in der Chirurgie beeinflussen.

Folgende Fragestellungen/Hypothesen wurden für die vorliegende Arbeit formuliert:

1. Es gibt hilfreiche und hinderliche Faktoren, welche die akademische Karriere in der Chirurgie beeinflussen.

2. Die Einflüsse auf eine erfolgreiche chirurgische Karriere unterscheiden sich zwischen Frauen und Männern.

3. Elternschaft beeinflusst die Karrieren von Chirurginnen und Chirurgen.

4. Es gibt Faktoren, die eine hohe berufliche Zufriedenheit ausmachen.

5. Frauen und Männer werden von unterschiedlichen Motiven geleitet, eine Karriere in der Chirurgie einzugehen. 


\section{Methoden}

\subsection{Auswahl der Stichprobe}

Über eine Onlinesuche erfolgte das deutschlandweite Screening nach habilitierten Chirurginnen auf den Webseiten aller deutschen Universitätskliniken, den dazugehörigen Lehrkrankenhäusern sowie Forschungsinstituten und der zum Teil vorhandenen Mitgliederlisten der Fachgesellschaften. Berücksichtigt wurden alle chirurgischen Fachgebiete, die in der Weiterbildungsordnung der Bundesärztekammer aufgelistet sind. Dazu gehören die Allgemein-, Gefäß-, Herz-, Kinder-, Trauma/Orthopädie-, Plastische-, Thorax-, und Viszeralchirurgie (Bundesärztekammer - Weiterbildungsordnung Chirurgie 2013). Um zu gewährleisten, dass die Teilnehmenden bereits eine fortgeschrittene berufliche Karriere absolviert hatten, wurden lediglich habilitierte Chirurginnen und Chirurgen zur Teilnahme an der Studie eingeladen (Einschlusskriterium). Chirurgen wurden in möglicher Kongruenz zu ihren weiblichen Kolleginnen ausgewählt, um zu gewährleisten, dass sich die Teilnehmenden in möglichst vielen relevanten soziodemographischen Faktoren wie Arbeitsort, Größe des Arbeitsplatzes, Fachgebiet und beruflicher Qualifikation ähneln. Dadurch wurde eine matched-pairDatenerhebung bereitet.

Um eine ähnliche Anzahl von Teilnehmenden in beiden Gruppen zu erhalten, wurden die Chirurginnen ( $n=93)$ und Chirurgen $(n=200)$ in einem 1:2- Verhältnis zur Studie eingeladen. Es war davon auszugehen, dass die Männer am Umfragethema weniger interessiert waren und seltener antworteten (eigene Beobachtungen).

\subsubsection{Datenerfassung}

Für diese Arbeit wurde der elektronische Weg der Datenerfassung über EvaSys ${ }^{\circledR}$ (Lüneburg, Deutschland) genutzt, einer Online-Software für Umfrageprozesse, um die Daten effizient und anonym zu erheben. Zwischen Juli und September 2014 erhielten die Teilnehmenden eine E-Mail mit einem Onlinelink, über den sie zum Fragebogen gelangten. Die Teilnehmenden, die noch nicht geantwortet hatten, erhielten in dreiwöchigen Abständen insgesamt drei Erinnerungen mit der Bitte zur Teilnahme. 
Nach Abschluss der Erhebung im September 2014 standen $n=63$ ausgefüllte Fragebögen von Chirurginnen und $n=70$ Fragebögen der Chirurgen als Daten zur Auswertung zur Verfügung. Die Teilnehmenden hatten die Möglichkeit, ihre E-MailAdresse für die Übermittlung der Studienergebnisse im Sinne einer vorläufigen Auswertung zur Verfügung zu stellen, die E-Mail-Adressen waren den ausgefüllten Fragebögen nicht zuzuordnen.

\subsection{Befragungsinstrument}

Das Erhebungsinstrument stellte ein 103 Items umfassender Fragebogen (siehe Anhang 6.1) dar.

Die Gliederung der Items erfolgte in den folgenden vier Kategorien:

1) Informationen zur Person und Berufstätigkeit: Alter, Fachbereich, berufliche Position, Bundesland, in dem sich der Arbeitsort befindet, Tätigkeitsfeld, Arbeitszeiten

2) beruflicher und familiärer Werdegang: Anzahl und Zeitpunkt der Geburt der Kinder, Kinderbetreuung, Motive der Berufswahl, förderliche und hinderliche interne und externe Faktoren und Bedingungen während des beruflichen Werdegangs

3) Freizeitgestaltung und Privatleben: Familienstand, Angaben zum beruflichen Arbeitsumfang des Partners oder der Partnerin, Freizeitgestaltung

4) Zufriedenheit und Selbsteinschätzung zu Karrierefaktoren: förderliche Eigenschaften für die Karriere, berufliche und private Zufriedenheit

Der Fragebogen beinhaltete festgelegte Antwortmöglichkeiten in Form von vier- und fünfstufigen Likert-Skalen, die von eins (positiv) bis vier bzw. fünf (negativ) reichten. Die berufliche und private Zufriedenheit wurde durch eine zehnstufige Likert-Skala erfasst. Außerdem bestand die Möglichkeit, freie Antworten sowie manuelle Zeit-und Prozentangaben zur Selbsteinschätzung zu geben. Die Fragetypen wurden simultan verwendet, um einem möglichen Verlust von Antworten vorzubeugen. Frauen und Männer wurden in separaten Kohorten zur Teilnahme an der Umfrage eingeladen, erhielten jedoch den gleichen Fragebogen, der bis auf minimale Änderungen der geschlechtsspezifischen Formulierungen nicht verändert wurde. Der Zeitaufwand zum Ausfüllen des Fragebogens betrug circa zehn Minuten. 


\subsection{Statistische Analyse}

Die Daten wurden elektronisch in das Statistikprogramm SPSS 22.0 (SPSS Inc., Chicago, Illinois) überführt. Die Freitextantworten wurden manuell in Microsoft Excel 2010 (Microsoft Inc., Redmond, Washington) übertragen und kategorisiert, sodass mehrere Freitextantworten einer Überkategorie zugeordnet wurden.

$\mathrm{Zu}$ Beginn der Berechnungen wurde geprüft, ob es sich bei den Daten um eine normalverteilte Grundgesamtheit handelt. Hierfür wurde der nicht-parametrische Test Kolmogorov-Smirnov-Test (kurz KS-Test) angewandt.

Die biografischen und beruflichen Daten der Teilnehmenden wurden deskriptiv analysiert, außerdem wurden folgende vier Subgruppen der Teilnehmenden miteinander verglichen:

- Chirurginnen versus Chirurgen,

- Kinderlose Teilnehmende versus Eltern,

- Mütter versus Väter,

- Berufliche Zufriedenheit $>90 \%$ versus $<90 \%$.

Es wurden Signifikanztests durchgeführt, um festzustellen, ob nicht zufallsbedingte Unterschiede in einer Subgruppen auftreten. Die Unterschiede der ordinalen Variablen wurden mittels Varianzanalyse (kurz: ANOVA) und t-Test analysiert. In dieser Arbeit wurden t-Tests und ANOVA mit einem Signifikanzniveau von $p<, 05$ für die Berechnung der ordinalen Variablen genutzt, da sich diese Tests auch bei nicht normalverteilten Daten als robust erwiesen haben und entsprechende Voraussetzungstests vernachlässigt werden können (Rasch und Guiard 2002; Rasch et al. 2009). Um sicherzugehen, wurden die Daten im Nachhinein mit dem parameterfreien Wilcoxon-Mann-Whithney-Test nachgerechnet. Die p-Werte änderten sich nicht, die relevanten Signifikanzen blieben unverändert.

Die nominalen Variablen wurden mit dem Chi-Quadrat-Test mit BonferroniAdjustierung verglichen. Aufgrund der Teilnehmerzahl wurde die Signifikanz mit dem Chi-Quadrat-Test nach Fisher berechnet. Bei einem p-Wert <,05 konnte von einem signifikanten Unterschied zwischen den Gruppen ausgegangen werden.

Um die Beziehung zwischen „hoher beruflicher Zufriedenheit“ als abhängige Variable und den deskriptiven Items (biografischen und beruflichen Daten, den Karrierefaktoren) als unabhängige Einflussvariablen (Prädiktoren) festzuhalten, 
wurde eine Regressionsanalyse durchgeführt. Mittels des R-Quadrats $\left(\mathrm{r}^{2}\right)$ konnte der Zusammenhang überprüft, durch den Beta-Koeffizienten die relative Wichtigkeit der Prädiktoren interpretiert werden. 


\section{Ergebnisse}

Nach Anwendung des KS-Tests musste von einer nicht-normalverteilten Grundgesamtheit ausgegangen werden $(p<, 05)$.

\subsection{Beschreibung der Stichprobe}

Tabelle 1 zeigt eine Zusammenfassung der deskriptiven Daten der Teilnehmenden. Der Fragebogen wurde an insgesamt 93 Frauen und 200 Männer gesendet, wovon n=63 (Rücklaufquote: 67.7\%) Frauen und n=70 (35\%) Männer antworteten.

\section{Tabelle 1: Deskriptive Daten}

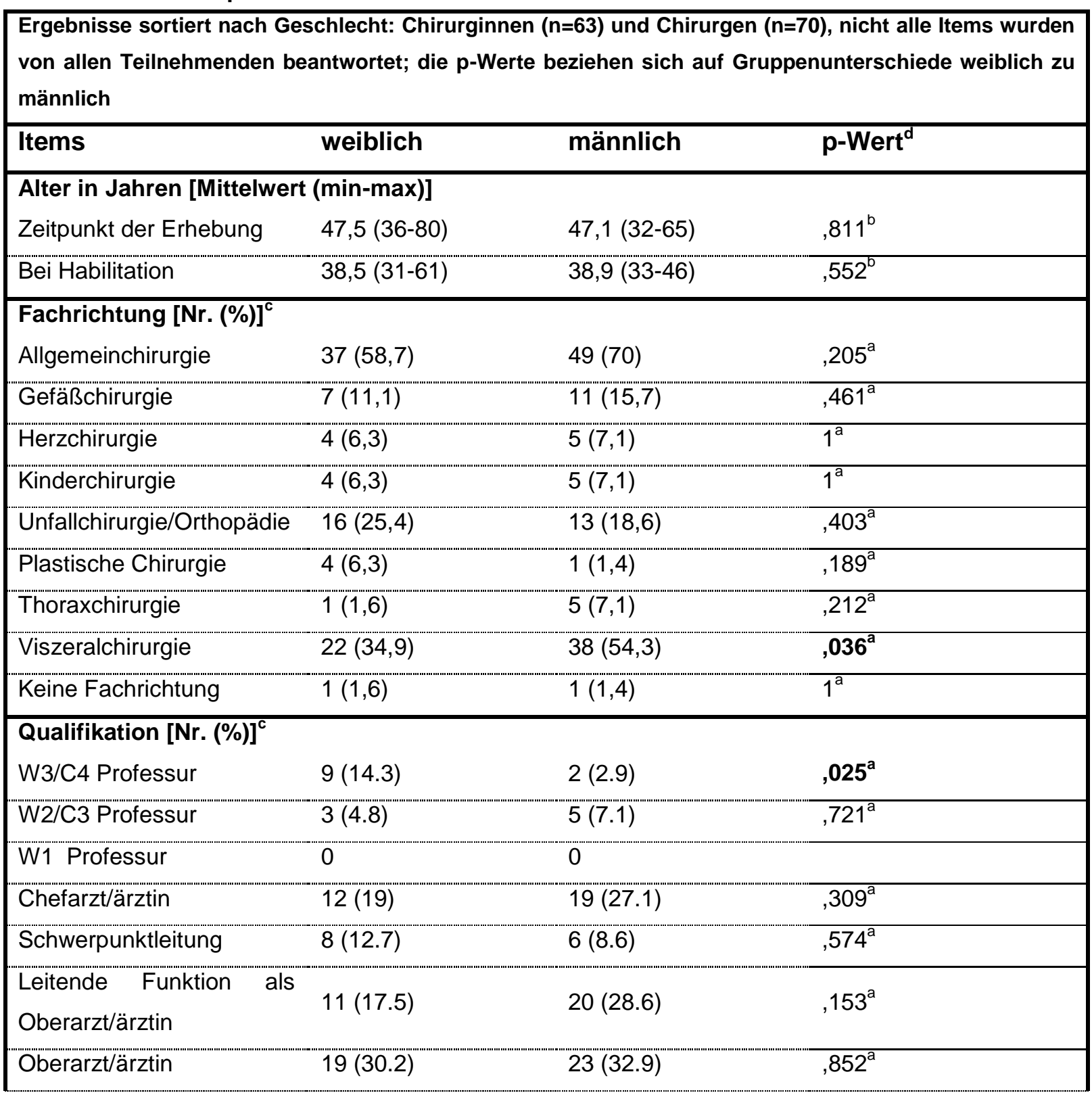




\begin{tabular}{|c|c|c|c|}
\hline Facharzt/ärztin & $5(7.9)$ & 0 &, $022^{\mathrm{a}}$ \\
\hline Assistenzarzt/ärztin & 0 & $1(1.4)$ & $1^{a}$ \\
\hline $\begin{array}{l}\text { Tätigkeitsfeld [Nr. (\%)] }^{\mathrm{C}} \\
\text { ambulante } \\
\text { Krankenversorgung/ } \\
\text { Praxis (angestellt) }\end{array}$ & $2(3.2)$ & $5(7.1)$ &, $445^{a}$ \\
\hline $\begin{array}{l}\text { ambulante } \\
\text { Krankenversorgung/ } \\
\text { Praxis (selbstständig) }\end{array}$ & $2(3.2)$ & 0 &, $222^{a}$ \\
\hline Elternzeit & $2(3.2)$ & 0 &, $222^{a}$ \\
\hline Forschungseinrichtung & $8(12.7)$ & $4(5.7)$ &, $227^{\mathrm{a}}$ \\
\hline keine Erwerbstätigkeit & $1(1.6)$ & 0 &, $474^{\mathrm{a}}$ \\
\hline $\begin{array}{l}\text { Krankenhaus (außer } \\
\text { Universitätsmedizin) }\end{array}$ & $19(30.2)$ & $26(37.1)$ &, $464^{a}$ \\
\hline Universitätsmedizin & $38(60.3)$ & $46(65.7)$ &, $590^{\mathrm{a}}$ \\
\hline Sonstiges & & &, $096^{a}$ \\
\hline \multicolumn{4}{|c|}{ wöchentliche Arbeitszeit in Stunden [Mittelwert (min-max)] } \\
\hline & $59,7(10-90)$ & $65.5(40-100)$ &, $008^{\mathrm{b}}$ \\
\hline \multicolumn{4}{|c|}{ Beschäftigungen in einer durchschnittlichen Arbeitswoche [Mittelwert in \%] $^{\mathrm{e}}$} \\
\hline Forschung & 15,1 & 9.1 &, $026^{\mathrm{b}}$ \\
\hline konservative Tätigkeiten & 25,4 & 18,1 &, $01^{D}$ \\
\hline Lehre & 11,4 & 7,9 & $0^{15}$ \\
\hline operative Tätigkeiten & 43,9 & 48,8 &, $144^{b}$ \\
\hline Verwaltung/Gremien & 14,3 & 15,1 & $664^{\mathrm{D}}$ \\
\hline $\begin{array}{l}\text { Familienstand [Nr. (\%)] } \\
\text { alleinstehend }\end{array}$ & $10(15.9)$ & $1(1.4)$ &, $003^{a}$ \\
\hline $\begin{array}{l}\text { verheiratet/in } \\
\text { Partnerschaft }\end{array}$ & $47(74.6)$ & $65(92.9)$ &, $004^{a}$ \\
\hline $\begin{array}{l}\text { geschieden/getrennt } \\
\text { lebend/ verwitwet }\end{array}$ & $6(9.5)$ & $4(5.7)$ &, $516^{\mathrm{a}}$ \\
\hline \multicolumn{4}{|l|}{ Anzahl der Kinder [Nr. (\%)] } \\
\hline Mittelwert & 1,47 & 2,24 &, $002^{\mathrm{b}}$ \\
\hline 0 & $14(22.6)$ & $8(13.8)$ &, $107^{\mathrm{a}}$ \\
\hline 1 & $22(35.5)$ & $9(15.5)$ &, $004^{\mathrm{a}}$ \\
\hline 2 & $14(22.6)$ & $17(29.3)$ &, $839^{a}$ \\
\hline 3 & $7(11.3)$ & $15(25.9)$ &, $160^{a}$ \\
\hline 4 & $5(8.1)$ & $7(12.1)$ &, $768^{\mathrm{a}}$ \\
\hline$>4$ & 0 & $2(3.4)$ & $498^{a}$ \\
\hline
\end{tabular}




\begin{tabular}{|c|c|c|c|}
\hline \multicolumn{4}{|c|}{ Alter bei Geburt des ersten Kindes [Mittelwert in Jahren (min-max)] } \\
\hline & $36,0(25-42)$ & $32,9(23-43)$ &, $002^{\mathrm{b}}$ \\
\hline \multicolumn{4}{|c|}{ die Kinderbetreuung in einer durchschnittlichen Woche ist verteilt auf [Mittelwert in \%] $^{\mathrm{e}}$} \\
\hline Ich selbst & 23,9 & 7,8 &, $001^{\mathrm{b}}$ \\
\hline Partnerln & 19,7 & 59,4 & $0^{b}$ \\
\hline $\begin{array}{l}\text { Betreuung im Haushalt } \\
\text { (Kindermädchen/AuPair) }\end{array}$ & 26,1 & 4,5 & $0^{b}$ \\
\hline $\begin{array}{l}\text { Einem anderem } \\
\text { Familienmitglied }\end{array}$ & 6,5 & 2,3 &, $457^{\mathrm{b}}$ \\
\hline Tagesmutter & 3,3 & 4 &, $872^{\mathrm{b}}$ \\
\hline Kindergarten /Schule/Hort & 40,70 & 30,3 &, $040^{\circ}$ \\
\hline Sonstiges & 20 & 1,75 &, $458^{b}$ \\
\hline \multicolumn{4}{|c|}{ Arbeitszeit des Partners in Stunden pro Woche [Mittelwert] } \\
\hline & 50,9 & 22,8 & $0^{\mathrm{b}}$ \\
\hline \multicolumn{4}{|c|}{$\begin{array}{l}\text { gegenwärtiges Zeitbudget einer durchschnittlichen Woche inkl. Wochenende } \\
\text { [Mittelwert in \%] }\end{array}$} \\
\hline Beruf & 66,4 & 68,6 &, $335^{b}$ \\
\hline Ehrenamt & 1,6 & 2,0 &, $557^{b}$ \\
\hline Familie & 19,9 & 18,5 &, $495^{b}$ \\
\hline Freizeitaktivitäten & 7,5 & 7,3 &, $800^{b}$ \\
\hline Haushalt & 7,4 & 5,9 &, $122^{b}$ \\
\hline Sonstiges & 5,0 & 2,2 &, $414^{b}$ \\
\hline \multicolumn{4}{|c|}{ Bildungsniveau der Eltern [Nr. (\%)] } \\
\hline beide akademisch & $25(41,0)$ & $26(37,1)$ &, $859^{\mathrm{a}}$ \\
\hline einer akademisch & $16(26,2)$ & $25(35,7)$ & $259^{\mathrm{a}}$ \\
\hline keiner & $20(32,8)$ & $19(27,1)$ &, $573^{\mathrm{a}}$ \\
\hline \multicolumn{4}{|c|}{ Zufriedenheit [Mittelwert in \%] } \\
\hline Arbeit & 69,5 & 75,7 &, $096^{\mathrm{b}}$ \\
\hline Leben & 76,8 & 80,8 &, $264^{b}$ \\
\hline \multicolumn{4}{|c|}{$\begin{array}{l}\text { a Chi-Quadrat- Kreuztabellen } \\
\text { b ANOVA } \\
{ }^{\text {c }} \text { Mehrfachantworten möglich } \\
\text { d signifikante } p \text {-Werte }(p<0,05) \text { sind in "fett" dargestellt } \\
\text { e die Prozentangaben sind subjektive Einschätzungen der Teilnehmer. Diese waren nicht verpflichtet, } \\
\text { auf } 100 \% \text { zu addieren }\end{array}$} \\
\hline
\end{tabular}


Im Schnitt waren die Teilnehmerinnen 47,5 Jahre. Die Jüngste der Chirurginnen war zum Zeitpunkt der Befragung 36 Jahre, die Älteste 80 Jahre alt. Die Teilnehmer waren im Durchschnitt 47,1 Jahre, mit einer Spannbreite von 32-65 Jahren. Mit etwa 28 Jahren erlangten beide Geschlechter den Doktorgrad, die Habilitation absolvierten die Teilnehmenden etwa 10 Jahre später mit 38,5 Jahren und 38,9 Jahren.

Die Mehrheit aller Teilnehmenden hatte die Facharztqualifikation für Allgemeinchirurgie erworben, der Anteil von Chirurgen in der Viszeralchirurgie fiel höher aus $(p<, 05)$. Die Teilnehmenden hatten die Möglichkeit, Mehrfachantworten zu geben und weitere Qualifikationen, Zusatzweiterbildungen oder Aufbaustudiengänge zu nennen. Die meistgenannten Zusatzweiterbildungen waren Notfallmedizin ( $n=15)$, Intensivmedizin $(n=12)$ und spezielle Unfallchirurgie $(n=11)$. Als Aufbaustudiengänge wurden u. a. der Master of Medical Education und der Master of Business Health Administration genannt (siehe Anhang 6.2.).

Zum Zeitpunkt der Befragung nahm ein höherer Anteil der Chirurginnen eine W3/C4Professur ein, gleichermaßen war der Anteil der Ärztinnen mit einer Facharztausbildung höher $(p<, 05)$. In Bezug auf die derzeitigen Beschäftigungsfelder der Teilnehmenden konnten keine signifikanten Unterschiede zwischen den Geschlechtern erhoben werden.

Die tatsächliche Arbeitszeit der Chirurginnen betrug durchschnittlich 59,7 Stunden pro Woche. Bei einer vertraglichen Arbeitszeit von 41,9 Stunden pro Woche reichte die tatsächliche Arbeitszeit von 10 bis 90 Stunden wöchentlich. Die Arbeitszeit der Männer betrug durchschnittlich 65,5 Stunden $(p<, 05)$. Die Arbeitsdauer erstreckte sich von mindestens 40 Stunden bis maximal 100 Stunden, bei einer vertraglich vereinbarten wöchentlichen Arbeitszeit von 42,2 Stunden.

Eine durchschnittliche Arbeitswoche verbrachten beide Geschlechter zu 43,9\% und 48,8 \% mit operativer Tätigkeit. Einen höheren Anteil ihrer Arbeitszeit investierten die Frauen in Forschung, konservative Tätigkeiten (z. B. Sprechstunden) und im Bereich der Lehre $(p<, 05)$. Für administrative Tätigkeiten spendeten Frauen und Männer etwa $15 \%$ ihrer wöchentlichen Arbeitszeit.

$31,7 \% \quad(n=20)$ der Chirurginnen und 22,6\% $(n=16)$ der Chirurgen hatten zum Zeitpunkt der Umfrage bereits einen 6-monatigen Forschungsaufenthalt absolviert. 
Bei Betrachtung des privaten Bereichs der Teilnehmenden fielen weitere Unterschiede auf. 74,6\% $(n=47)$ der Chirurginnen waren verheiratet oder lebten in einer Partnerschaft, bei den Männern waren es 92,9\% $(n=65)(p<, 05)$.

$15,9 \%(n=10)$ der Chirurginnen lebten in keiner Partnerschaft und hoben sich von ihren männlichen Kollegen ab, von denen $1,4 \%(n=1)$ allein lebten. Geschieden oder verwitwet waren jeweils weniger als $10 \%$ aller Teilnehmenden.

Die Partnerinnen und Partner der Chirurginnen arbeiteten im Schnitt 50,9 Stunden, die der Männer 22,8 Stunden pro Arbeitswoche $(p<, 05)$.

Ein Großteil der Partnerinnen und Partner war im medizinischen Bereich tätig. Neun der Partnerinnen und Partner der Chirurgen waren aktuell nicht berufstätig, die Partnerinnen und Partner der Chirurginnen befanden sich alle in einer beruflichen Beschäftigung (siehe Anhang 6.3).

Unter den Chirurginnen befanden sich 77,5\% $(n=48)$ Mütter mit durchschnittlich 1,47 Kindern. Bei den männlichen Teilnehmern waren 86,2\% $(n=50)$ Väter, mit durchschnittlich 2,24 Kindern $(p<, 05)$. Die Mütter bekamen ihr erstes Kind mit durchschnittlich 36 Jahren, die Väter waren bei der Geburt 32,9 Jahre. Der Großteil $(41,4 \%, n=29)$ der Väter befand sich bei der Geburt des ersten Kindes in der fachärztlichen Weiterbildung, von den Frauen bekamen die meisten ihr erstes Kind zu einem späteren beruflichen Zeitpunkt $(p<, 05)$.

Bei den Aufgaben der Kinderbetreuung ließen sich signifikante Unterschiede zwischen den Geschlechtern feststellen. Während 26,1\% der Chirurginnen die Kinderbetreuung an eine Betreuung im Haushalt (Nanny, Au-Pair, Kinderfrau) übergaben, 23,9 \% die Betreuung selbst übernahmen und bei $19,7 \%$ die Kinderbetreuung durch den Partner oder Partnerin erfült wurde, wurde bei den Chirurgen 59,4\% der Kinderbetreuung durch die Partnerin oder den Partner übernommen $(p<, 05)$. Lediglich $4,5 \%$ wurden durch eine Betreuung im Haushalt (Nanny, Au-Pair, Kinderfrau) und 7,8\% der Kinderbetreuung durch den Mann selbst übernommen ( $p<, 05$ ). 40,7\% der Frauen und 30,3\% der Männer übergaben dem Kindergarten/Hort oder der Schule die Betreuung der Kinder $(p<, 05)$.

Die mittlere Arbeitszufriedenheit war bei beiden Geschlechtern hoch. Bei der allgemeinen Lebenszufriedenheit gaben die Frauen $(76,8 \%)$ eine etwas geringere mittlere Zufriedenheit an als die Männer (80,8\%). 
Zusammenfassend kann gesagt werden, dass die durchschnittliche Teilnehmerin zum Zeitpunkt der Umfrage 47,5 Jahre alt war und die Habilitation mit 38,5 Jahren abschloss. Die Mehrzahl der Teilnehmerinnen befand sich als Fachärztin für Allgemeinchirurgie in einer Position als Oberärztin an einem Universitätskrankenhaus. Die Hauptarbeitszeit verbrachte sie mit operativen Tätigkeiten. Die meisten der Teilnehmerinnen waren verheiratet oder in einer Partnerschaft mit durchschnittlich 1,47 Kindern, die Betreuung dieser übernahm hauptsächlich eine externe Kinderbetreuungsstätte.

Der durchschnittliche Teilnehmer war bei Teilnahme 47,1 Jahre alt und legte die Habilitation mit 38,9 Jahren ab. Die Hauptanzahl der Teilnehmer war ebenfalls als Facharzt für Allgemeinchirurgie in einer Oberarztposition an einem Universitätskrankenhaus tätig. Auch die Männer verbrachten die Hauptarbeitszeit mit operativen Tätigkeiten. Die meisten lebten in einer Partnerschaft und hatten 2,24 Kinder, die zum größten Teil durch die Partnerin oder den Partner betreut wurden. Alle Teilnehmenden schätzten sowohl ihre berufliche, als auch ihre Lebenssituation als sehr zufriedenstellend ein.

\subsection{Geschlechtsabhängiger Einfluss auf eine chirurgische Karriere}

\subsubsection{Bewertung der Motive für die Wahl der Weiterbildung in der Chirurgie}

In Abbildung 3 werden die Bewertungen der Chirurginnen und Chirurgen zu den Motiven für ihre Berufswahl verglichen. 


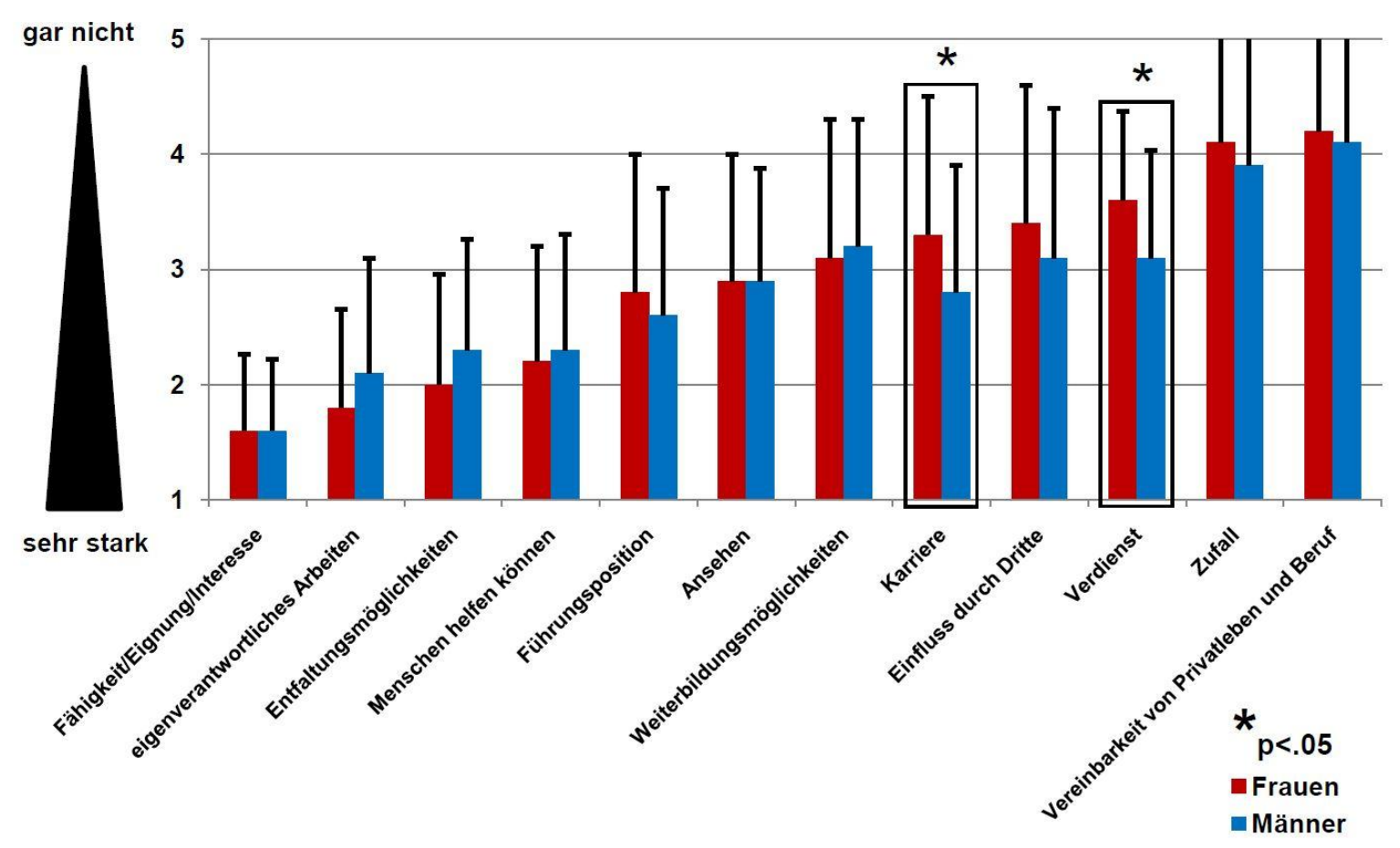

\section{Abbildung 3: Motive für die Wahl zur Weiterbildungsdisziplin Chirurgie}

Ergebnisse in aufsteigender Reihe, sortiert nach Geschlecht; nicht alle Items wurden von allen Teilnehmenden beantwortet, die Likert-Skala reicht von eins (sehr stark) bis fünf (gar nicht). Die Darstellung erfolgt als Mittelwert und Standardabweichung.

Für beide Geschlechter (Frauen vs. Männer) war das Motiv „Fähigkeit/Eignung/Interesse“ besonders wichtig für die Berufswahl, gefolgt vom „eigenverantwortlichen Arbeiten“ und von „Entfaltungsmöglichkeiten“. „Zufall“ und die „Vereinbarkeit von Beruf und Privatleben“ war für beide Gruppen am wenigsten motivierend für die Berufswahl. Für beide Geschlechter bedeuteten die Items „Verdienst“ und „Karriere“ eine geringe Motivation für die Berufswahl, wobei Frauen diese Motive als noch unwichtiger einschätzen $(p<, 05)$.

Auf die offene Frage nach sonstigen Gründen, eine Karriere in der Chirurgie einzuschlagen, betonten viele Teilnehmende das breite, abwechslungsreiche Spektrum der Arbeit. Unterstrichen wurde, dass es sich um eine „wenig schreibtischlastige Tätigkeit" handele. Für zwei Teilnehmende sei die Chirurgie „Schon immer der Traumberuf“ gewesen. Hier könne man „durch sein Dasein einen Unterschied bewirken“. Die Motivation, etwas Handwerkliches zu leisten, sowie der „Spaß am akademischen Arbeiten“ wurden ebenfalls gesondert genannt. Der Einfluss durch Dritte in Form von „Interessensbildung während des Studiums“ sowie 
der „Zivildienst“ wurden als Einflussfaktoren hervorgehoben, ebenso die Vorbildfunktion ehemaliger Vorgesetzter (man wolle „sein wie er“). Da Chirurginnen und Chirurgen hier weitestgehend ähnliche Antworten gaben, wurde auf eine Geschlechtertrennung verzichtet.

Zusammenfassend motivierten persönliche Fähigkeiten und das Streben nach beruflicher Interessenverwirklichung die Teilnehmenden, den chirurgischen Berufsweg einzuschlagen. Ausreichend Möglichkeiten für Freizeitgestaltung nahmen einen geringeren Stellenwert ein, ebenso ein finanzieller Anreiz oder Karrieremöglichkeiten.

\subsubsection{Bewertung von Karrierefaktoren}

Abbildung 4 zeigt die Einschätzungen der Teilnehmenden zu einer Auswahl an Karrierefaktoren für eine erfolgreiche akademische Karriere.

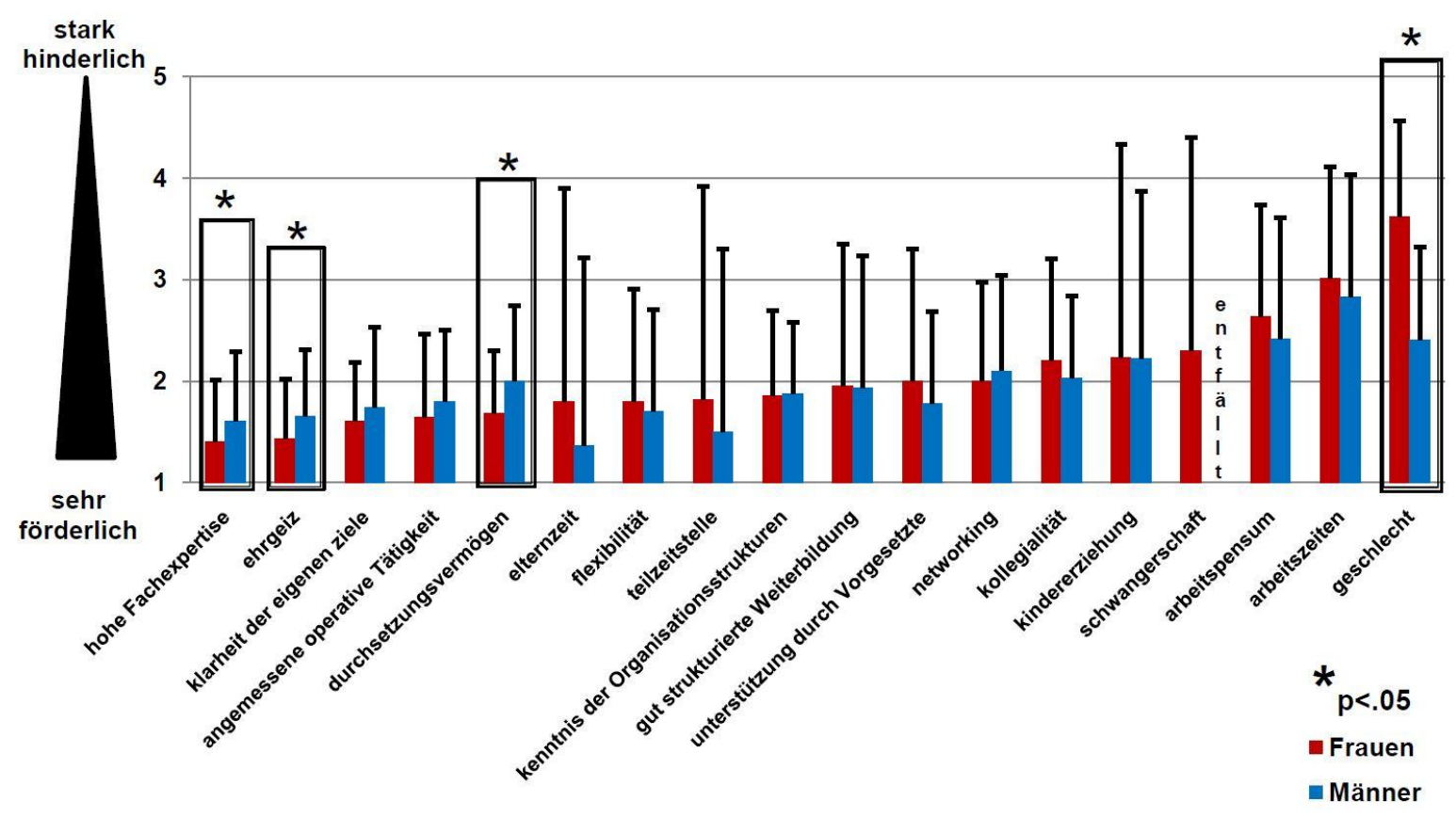

\section{Abbildung 4: Faktoren für die Karriereentwicklung}

Ergebnisse in aufsteigender Reihe, sortiert nach Geschlecht; nicht alle Items wurden von allen Teilnehmenden beantwortet, die Likert-Skala reicht von eins (sehr stark) bis fünf (gar nicht). Die Darstellung erfolgt als Mittelwert und Standardabweichung.

Beide Geschlechter (Frauen vs. Männer) evaluierten eine "hohe Fachexpertise“, „Ehrgeiz" sowie die „Klarheit der eigenen Ziele“ als förderliche Faktoren für ihren 
beruflichen Aufstieg. Die beiden erstgenannten Items stuften die Chirurginnen als förderlicher für ihre Karriere ein $(p>, 05)$. Ebenso wurde von den Frauen „Durchsetzungsvermögen" als wichtiger empfunden $(p<, 05)$.

Am hinderlichsten erachteten die Teilnehmenden das "Arbeitspensum", die „Arbeitszeit" sowie das "Geschlecht", letzteres empfanden die Frauen noch hinderlicher $(\mathrm{p}<, 05)$.

Tabelle 2 führt Beispiele der offenen Anmerkungen und Kommentare zu Karrierefaktoren auf. Aus Gründen der Übersicht wurden die Antworten in semantische Kategorien gegliedert und in Rechtschreibung korrigiert.

Tabelle 2: Freitextantworten zu Karrierefaktoren

\begin{tabular}{|c|c|c|}
\hline Kategorie & Anzahl[Nr.] & Exemplarische Antworten \\
\hline Strukturänderung & 4 & $\begin{array}{l}\text { - „Änderung des } \\
\text { Mutterschutzgesetzes: } \\
\text { „(...)Schwangerschaft nicht } \\
\text { gemeldet wegen Problemen in } \\
\text { Deutschland (...)“ } \\
\text { - "Vereinbarkeit von Forschung und } \\
\text { Klinik“ } \\
\text { - „Aufbrechen alter hierarchischer } \\
\text { Strukturen“ }\end{array}$ \\
\hline Organisation & 5 & $\begin{array}{l}\text { - "Perspektivenklarheit" } \\
\text { - "Sprachtraining, falls kein } \\
\text { Auslandaufenthalt" } \\
\text { "Ausbau und Vertiefung des } \\
\text { Netzwerkes }(. . .)^{\text {" }}\end{array}$ \\
\hline eigenes Auftreten & 5 & $\begin{array}{ll}\text { - } & \text { "Disziplin“ } \\
\text { - } & \text { "intrinsische Motivation“ } \\
\text { - } & \text { „offene Kommunikation(...)“ }\end{array}$ \\
\hline
\end{tabular}

Es erfolgte eine Bezugnahme auf nötige Strukturänderungen, wie z. B. eine Änderung des Mutterschutzgesetzes und eine bessere Organisation des Arbeitsumfeldes. Überdies wurde die Relevanz des eigenen Auftretens, beispielsweise in Form von Disziplin oder persönlichem Einsatz betont.

Zusammenfassend erachteten die Teilnehmenden Attribute als förderlich für eine berufliche Karriere, die mit Persönlichkeitsstärke assoziiert sind. Zudem nahmen berufliche Fertig- und Fähigkeiten einen positiven Einfluss auf den Beruf. Der Arbeitsumfang in Kombination mit zu vielen Diensten belastete die Teilnehmenden. 
Es wurde eine bessere Verknüpfung von Klinik und Forschung und eine organsiertere Laufbahnberatung gefordert. Noch immer stellte das weibliche Geschlecht einen Nachteil im beruflichen Aufstieg dar.

\subsubsection{Bewertung von Persönlichkeitsmerkmalen}

Abbildung 5 zeigt die Einschätzungen der Teilnehmenden von intrinsischen Charakteristika und Persönlichkeitsmerkmalen während der beruflichen Karriere

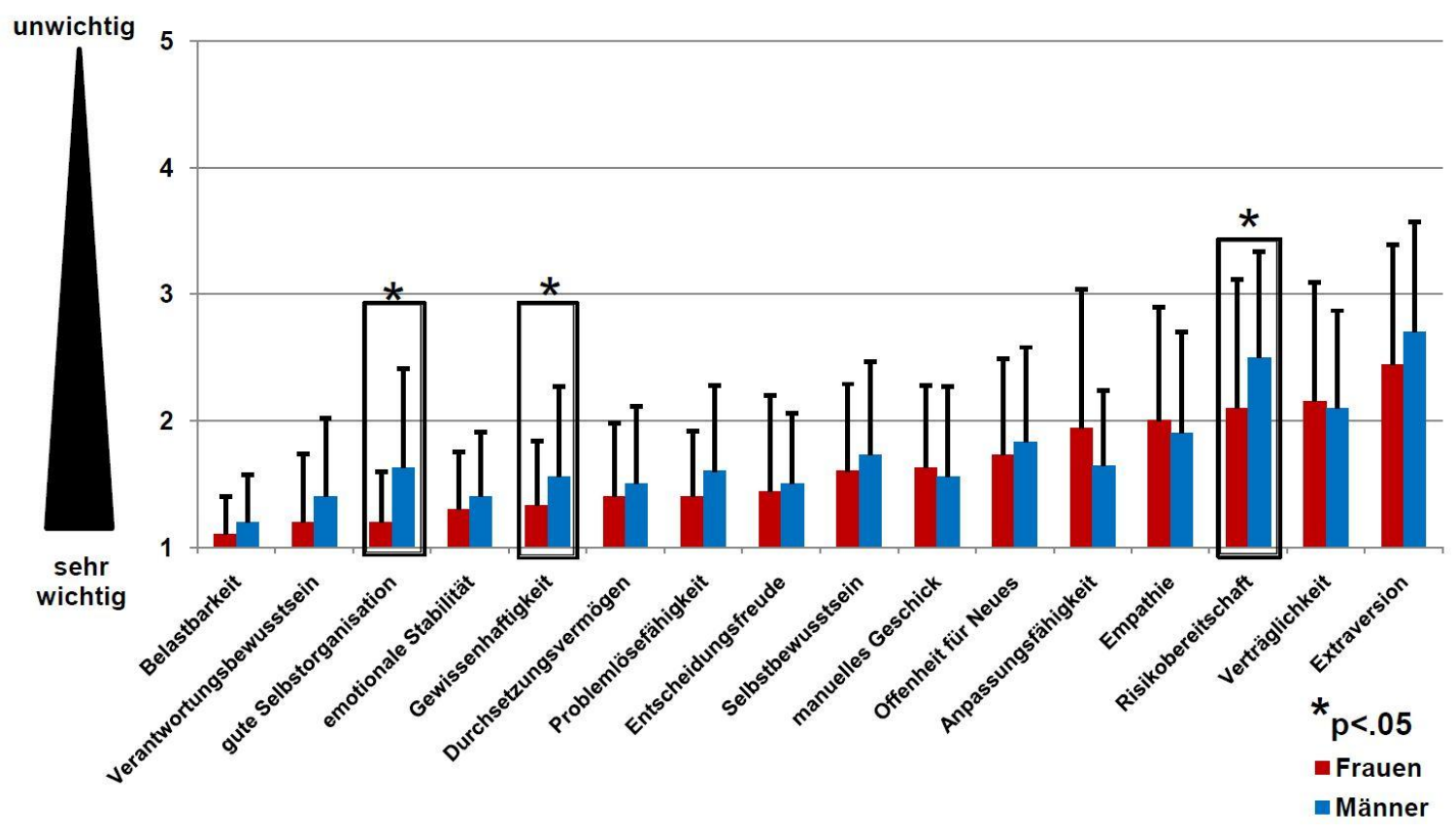

\section{Abbildung 5: Förderliche Eigenschaften für die Karriereentwicklung}

Ergebnisse in aufsteigender Reihe, sortiert nach Geschlecht; nicht alle Items wurden von allen Teilnehmenden beantwortet, die Likert-Skala reicht von eins (sehr stark) bis fünf (gar nicht). Die Darstellung erfolgt als Mittelwert und Standardabweichung.

Die Items wurden in Orientierung an das Fünf-Faktoren-Modell (Goldberg 1990) erstellt. Dieses Modell beschreibt die Persönlichkeit in folgenden fünf Kategorien: Neurotizismus, Extraversion, Offenheit, Gewissenhaftigkeit und Verträglichkeit. In dieser Arbeit wurde statt der Formulierung Neurotizimus die bedeutungsgleiche Formulierung „emotionale Stabilität“ verwendet, um mehr Verständlichkeit zu vermitteln.

Beide Geschlechter (Frauen versus Männer) schätzten „Belastbarkeit", „Verantwortungsbewusstsein" und eine "gute Selbstorganisation" als die wichtigsten Eigenschaften für einen beruflichen Aufstieg ein. Frauen bewerteten die Items „gute 
Selbstorganisation“, „Gewissenhaftigkeit“ sowie „Risikobereitschaft“ als wichtiger für den beruflichen Aufstieg ( $\mathrm{p}<, 05)$.

Im Hinblick auf das Fünf-Faktoren-Modell waren die Items im Ranking ohne Häufung verteilt. „Emotionale Stabilität“ und „Gewissenhaftigkeit“ lagen auf Platz vier und fünf von insgesamt 16 Items. Im unteren Viertel lag „Offenheit für Neues“. Als die unwichtigsten Eigenschaften für einen beruflichen Aufstieg wurden „Verträglichkeit“ und „Extraversion“ genannt, allerdings wurde kein Item schlechter bewertet als mit einem durchschnittlichen Mittelwert von 2,7.

Tabelle 3 zeigt die Freitextantworten der Teilnehmenden auf die Frage, ob es ihrer Meinung nach erfolgsrelevante Eigenschaften gäbe, die bei dem jeweils anderen Geschlecht stärker ausgeprägt seien. Aus Gründen der Übersicht wurden die Antworten in semantische Kategorien gegliedert und in Rechtschreibung korrigiert.

Tabelle 3: Freitextantworten zu geschlechtsspezifischen erfolgsrelevanten Eigenschaften

\begin{tabular}{|c|c|c|}
\hline Kategorien & $\begin{array}{l}\text { Anzahl } \\
\text { [Nr.] }\end{array}$ & Exemplarische Antworten \\
\hline Effektivität im Beruf & 10 & $\begin{array}{ll}\text { - } & \text { „Durchsetzungsfähigkeit“ } \\
\text { - } & \text { "Planungsfähigkeit“" } \\
\text { - } & \text { "Fleißiger, ohne darüber zu sprechen } \\
& \text { (führt aber nicht zu } \\
& \text { Anerkennung/Erfolg)“ } \\
\text { - } & \text { "Zielstrebigkeit“ } \\
\text { - } & \text { "gutes Fachwissen“ }\end{array}$ \\
\hline Fünf-Faktoren-Modell & 4 & $\begin{array}{l}\text { - ,fehlende Verträglichkeit" } \\
\text { - "emotionale Instabilität“ } \\
\text { - "Gewissenhaftigkeit“" }\end{array}$ \\
\hline Sozialer Umgang & 3 & $\begin{array}{l}\text { - "fehlendes Einfühlungsvermögen“ } \\
\text { - "Empathie“ } \\
\text { - " „Soziale Kompetenz" }\end{array}$ \\
\hline Teamfähigkeit & 2 & $\begin{array}{l}\text { - } \quad \text { "Loyalität } \\
\text { - "Kollegialität } t^{\prime \prime}\end{array}$ \\
\hline $\begin{array}{l}\text { keine Unterschiede } \\
\text { vorhanden }\end{array}$ & 15 & - „Nein“ \\
\hline Egoismus & 14 & 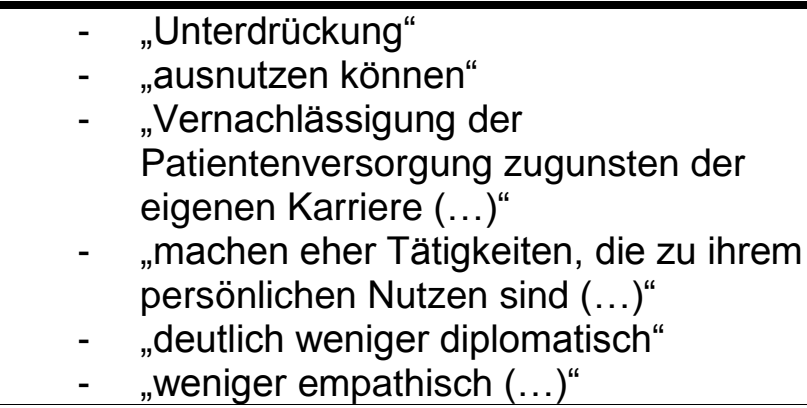 \\
\hline
\end{tabular}




\begin{tabular}{|c|c|c|}
\hline Führungsfähigkeit & 8 & $\begin{array}{ll}\text { - } & \text { "(...) mehr Alphatier-Persönlichkeit“ } \\
\text { - } & \text { "Entscheidungsfreude“ } \\
\text { - } & \text { "Organisationstalent“ } \\
\text { - } & \text { "Verantwortungsbewusstsein“ } \\
\text { - } & \text { "direkt: sehr frontal“ }\end{array}$ \\
\hline Karriereplanung & 2 & - "langfristige Ziele besser im Auge“ \\
\hline Networking & 6 & - „Männerclubbildung“ \\
\hline $\begin{array}{l}\text { Keine Unterschiede } \\
\text { vorhanden }\end{array}$ & 2 & \\
\hline Präsenz & 3 & $\begin{array}{ll}\text { - } & \text { „Männlichkeit an sich (...)“ } \\
\text { - } \quad \text { „lange Anwesenheiten“ }\end{array}$ \\
\hline Risikobereitschaft & 7 & $\begin{array}{ll}\text { - } & \text { "(...)bis zur Verantwortungslosigkeit“ } \\
\text { - } & \text { "geringere Gewissenhaftigkeit“" } \\
\text { - } & \text { „Kaltblütigkeit“ }\end{array}$ \\
\hline Selbstsicherheit & 18 & $\begin{array}{ll}\text { - } & \text { "Größenwahn“ } \\
\text { - } & \text { "Selbstherrlichkeit“ } \\
\text { - } & \text { „mehr Eigenwerbung }(. . .) \text { “ } \\
\end{array}$ \\
\hline Zielstrebigkeit & 12 & $\begin{array}{ll}\text { - } & \text { „Durchhaltevermögen, trotz negativer } \\
\text { Erfahrungen“ } \\
\text { - } \quad \text { (...) den Wille Karriere zu machen(...) } \\
\text { - } \quad \text { „Narzissmus“ }\end{array}$ \\
\hline
\end{tabular}

Die Chirurgen schrieben im Allgemeinen sehr positive Anmerkungen über ihre weiblichen Kolleginnen. Sie hatten den Eindruck, ihre Kolleginnen seien u. a. „durchsetzungsfähiger“, „kollegialer“, „empathischer“, „zielstrebiger“ und verfügten über ein "gutes Fachwissen“. An negativ-behafteten Eigenschaften wurde von den Männern u. a. „fehlendes Einfühlungsvermögen“, „fehlende Verträglichkeit“ sowie „emotionale Instabilität" genannt.

Die Chirurginnen gaben eine größere Anzahl an Kommentaren bei dieser Frage ab. Zusammenfassend sahen die Chirurginnen ihre männlichen Kollegen als „konfliktbereiter", „selbstsicherer", „risikofreudiger", „egoistischer" und „zielstrebiger" an. Außerdem besäßen die Chirurgen „mehr Führungsqualitäten", verfügten über eine „bessere Karriereplanung" und ein „breiteres Netzwerk".

Hervorgehoben wurde, dass die "Männlichkeit an sich" schon ein Vorteil gegenüber Frauen sei und "lange Anwesenheiten" in der Klinik Vorteile mit sich bringe. Die Chirurginnen würden sich häufig erst um „Station und OP" kümmern und dann um die eigene Karriere. 
Zusammenfassend beurteilten die Teilnehmenden die Charakterzüge, die Stärke, Belastbarkeit, Organisation und Zuverlässigkeit ausstrahlen, als wichtig für die berufliche Laufbahn. Frauen empfanden ihre männlichen Kollegen als egoistischer und begabter, den Arbeitsalltag mehr zu ihren Gunsten zu organisieren. Bessere Führungsqualitäten und ihr Geschlecht seien innen dabei behilflich. Die Männer nahmen ihre weiblichen Kolleginnen im Allgemeinen als sorgfältiger und pflichtbewusster wahr; trotz ausgebauter sozialer Kompetenz hätten die Frauen allerdings weniger Erfolg im Beruf. Zudem wurde den Frauen durch die Männer eine emotionale Instabilität zugeschrieben.

\subsection{Geschlechtsunabhängiger Einfluss auf eine chirurgische Karriere}

\subsubsection{Bewertung karrierebeeinflussender Maßnahmen im Arbeitsumfeld}

Die Teilnehmenden hatten die Möglichkeit anzugeben, ob ausgewählte Angebote oder Arbeitsvoraussetzungen genutzt wurden und ob dieses Angebot als hilfreich oder nicht hilfreich empfunden wurde. Außerdem konnte eine Angabe zur Verfügbarkeit der Angebote gemacht werden (Nicht verfügbar/Nicht notwendig).

In Tabelle 4 sind die Antworten der Chirurginnen und Chirurgen dargestellt. Diese wurden in Kategorien gegliedert, die Anzahl der Antworten wurde auf ganze Prozente gerundet. Es waren keine signifikanten Geschlechtsunterschiede feststellbar, sodass auf einen Gruppenvergleich (Frauen versus Männer) verzichtet wurde.

Tabelle 4: Karrierebeeinflussende Maßnahmen im Arbeitsumfeld

Es konnten keine signifikanten Unterschiede zwischen den Geschlechtern nach Anwendung
von Signifikanztests festgestellt werden. Die Anzahl der Antworten wurde auf ganze
Prozente gerundet, nicht alle Items wurden von allen Teilnehmenden beantwortet




\begin{tabular}{|c|c|c|c|c|}
\hline & nicht genutzt & $73(56)$ & $\begin{array}{l}\text { nicht vorhanden } \\
\text { kein Bedarf }\end{array}$ & $\begin{array}{l}65(89) \\
8(11)\end{array}$ \\
\hline & entfällt & 16(12) & & \\
\hline \multirow{5}{*}{$\begin{array}{l}\text { erleichterter Wiedereinstieg } \\
\text { nach der Elternzeit }\end{array}$} & genutzt & 12(9) & hilfreich & $\mathbf{9}(75)$ \\
\hline & & & nicht hilfreich & $3(25)$ \\
\hline & nicht genutzt & $64(50)$ & nicht vorhanden & $38(59)$ \\
\hline & & & kein Bedarf & 26(41) \\
\hline & entfällt & $53(41)$ & & \\
\hline \multirow[t]{5}{*}{ Teilzeitarbeitsmodelle } & genutzt & $14(11)$ & hilfreich & 11(79) \\
\hline & & & nicht hilfreich & $3(21)$ \\
\hline & nicht genutzt & $71(55)$ & nicht vorhanden & $44(62)$ \\
\hline & & & kein Bedarf & $27(38)$ \\
\hline & entfällt & 44(34) & & \\
\hline \multirow[t]{5}{*}{ Karriereplanungen } & genutzt & $77(58)$ & hilfreich & $\mathbf{5 4}(70)$ \\
\hline & & & nicht hilfreich & $23(30)$ \\
\hline & nicht genutzt & $44(33)$ & nicht vorhanden & $32(73)$ \\
\hline & & & kein Bedarf & 12(27) \\
\hline & entfällt & 12(9) & & \\
\hline \multirow[t]{5}{*}{ Mentoring } & genutzt & $56(42)$ & hilfreich & $38(68)$ \\
\hline & & & nicht hilfreich & $18(32)$ \\
\hline & nicht genutzt & $66(50)$ & nicht vorhanden & $\mathbf{5 0}(76)$ \\
\hline & & & kein Bedarf & $16(24)$ \\
\hline & entfällt & $11(8)$ & & \\
\hline \multirow[t]{5}{*}{ Supervision } & genutzt & $35(26)$ & hilfreich & $25(71)$ \\
\hline & & & nicht hilfreich & 10(29) \\
\hline & nicht genutzt & $82(62)$ & nicht vorhanden & $66(80)$ \\
\hline & & & kein Bedarf & $16(20)$ \\
\hline & entfällt & $16(12)$ & & \\
\hline \multirow{5}{*}{$\begin{array}{l}\text { Personalentwicklung/ } \\
\text { Schlüsselkompetenzen }\end{array}$} & genutzt & $46(34)$ & hilfreich & 31(67) \\
\hline & & & nicht hilfreich & $15(33)$ \\
\hline & nicht genutzt & $74(56)$ & nicht vorhanden & $58(78)$ \\
\hline & & & kein Bedarf & $16(22)$ \\
\hline & entfällt & $13(10)$ & & \\
\hline \multirow[t]{5}{*}{ Nachwuchsförderung } & genutzt & $47(36)$ & hilfreich & $38(81)$ \\
\hline & & & nicht hilfreich & $\mathbf{9}(19)$ \\
\hline & nicht genutzt & 66(51) & nicht vorhanden & $58(88)$ \\
\hline & & & kein Bedarf & $8(12)$ \\
\hline & entfällt & $17(13)$ & & \\
\hline
\end{tabular}


Nahezu die Hälfte der Teilnehmenden (48\%) hatte kein Angebot zu „Betreuungsangebote für die eigenen Kinder“ genutzt, da in $69 \%$ der Fälle kein Angebot zur Verfügung stand.

$32 \%$ der Chirurginnen und Chirurgen hatten die Möglichkeit, sich auf „Arbeits- und Urlaubsplanungen“ zu verlassen, 55\% von diesen empfanden die Organisation als hilfreich. 56\% der Teilnehmenden hatte keinen Zugang zu verlässlichen „Arbeits- und Urlaubsplanungen“, da diese in 89\% der Fälle nicht zur Verfügung standen.

Einen erleichterten „Wiedereinstieg nach der Elternzeit“ und „Teilzeitarbeitsmodelle“ wurde in 50\% und 55\% der Fälle nicht genutzt. In jeweils etwa $40 \%$ der Fälle empfanden die Teilnehmenden beide Angebote als nicht notwendig.

Angebote zur „Karriereplanung“ konnten 58\% der Teilnehmenden in Anspruch nehmen, 70\% von diesen empfanden das Angebot als hilfreich. „Mentoring“ und „Supervision“ wurden in 50\% und $62 \%$ nicht genutzt, weil es in jeweils $20 \%$ der Fälle als nicht notwendig empfunden wurde. 56\% und 51\% der Teilnehmenden konnte weder von Angeboten zur „Personalentwicklung“, noch von Veranstaltungen zur „Nachwuchsförderung“ Gebrauch machen, weil sie in 78\% und $88 \%$ der Fälle nicht verfügbar waren. Es bestand die Möglichkeit in Freitextantworten anzugeben, welche weiteren Unterstützungen sich die Teilnehmenden von ihren Arbeitgebern wünschen würden. Die Antworten wurden kategorisiert und in Rechtschreibung korrigiert.

Tabelle 5: Freitextantworten zu wünschenswerten Veränderungen im Arbeitsumfeld

$\mathrm{N}=46$, davon $\mathrm{n=34}$ Frauen und n=12 Männer, die Antworten der Männer sind „kursiv“ dargestellt

\begin{tabular}{|c|c|c|}
\hline $\begin{array}{l}\text { Kategorie des } \\
\text { Verbesserungsvorschlags }\end{array}$ & $\begin{array}{l}\text { Anzahl } \\
\text { [Nr.] }\end{array}$ & Exemplarische Antworten \\
\hline $\begin{array}{l}\text { Arbeits-\& Familien/- } \\
\text { Lebens Vereinbarkeit }\end{array}$ & 14 & 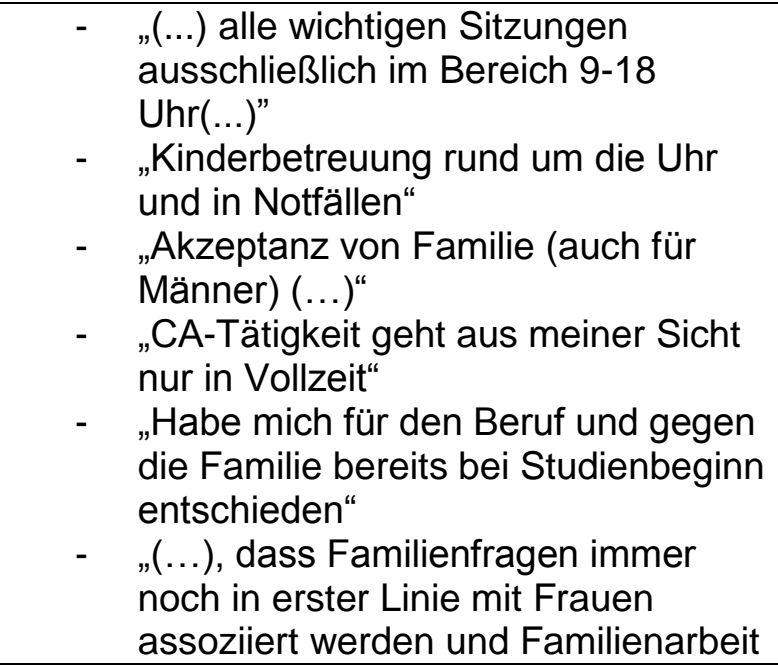 \\
\hline
\end{tabular}




\begin{tabular}{|c|c|c|}
\hline & & $\begin{array}{ll}\text { durch Männer wenig akzeptiert ist.“ } \\
\text { - } \\
\text { „Erhöhung der Anzahl der KITA } \\
\text { Plätze + Kindergarten” } \\
\text { - } \quad \text { „angepasste Ausbildungspläne“ }\end{array}$ \\
\hline Geschlechtergleichheit & 5 & $\begin{array}{ll}\text { - } & \text { „(...) mehr Achten auf tatsächliche } \\
\text { Gleichstellung, z. B. fester } \\
\text { Prozentsatz an Frauen in politisch- } \\
\text { strategisch wichtigen Gremien (...)” } \\
\text { - } \quad \text { "Transparenz der Aufstiegschancen” } \\
\text { - } \quad \text { (...)bin als habilitierte Allgemein- } \\
\text { und Viszeralchirurgin deutlich höher } \\
\text { qualifiziert als einer der mir } \\
\text { vorgesetzten Oberärzte” }\end{array}$ \\
\hline $\begin{array}{l}\text { Institutionelle } \\
\text { Unterstützung }\end{array}$ & 23 & 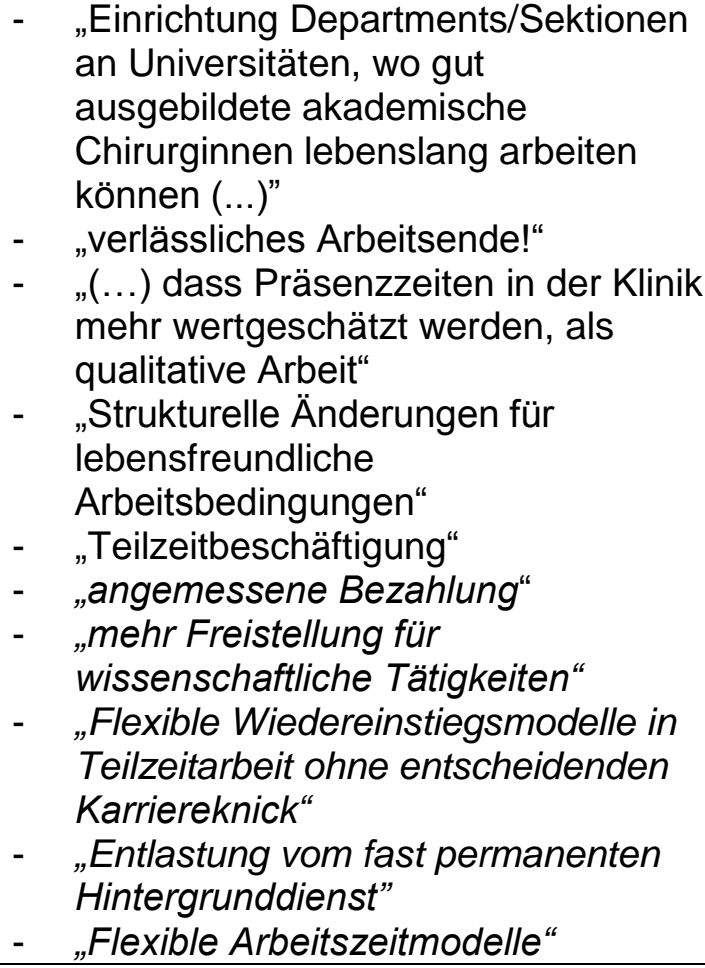 \\
\hline $\begin{array}{l}\text { Führungs-und } \\
\text { Karriereentwicklung }\end{array}$ & 4 & $\begin{array}{ll}\text { - } & \text { „Mentoring” } \\
\text { - } & \text { „Supervision” } \\
\text { - } & \text { "(...) Fortbildungen für männliche } \\
& \text { Führungskräfte }(. . .)^{\circ} \\
\text { - } & \text { „Fortbildung soziale Kompetenzen“ }\end{array}$ \\
\hline
\end{tabular}

Auf Basis der Antworten konnten vier Kategorien erstellt werden. Die Antworten basierten auf der Thematik von Arbeits-/Familienvereinbarkeit, Geschlechtergleichheit, institutioneller Unterstützung und Führungs- und Karriereentwicklung. Außerdem wurde explizit darauf hingewiesen, dass die akademische Chirurgie in Teilzeit als nicht sinnvoll erachtet werde und man sich deswegen bei Beginn des Studiums bereits gegen Familie entschieden habe. 
Zusammenfassend hatten die Teilnehmenden selten die Möglichkeit, unterstützende Maßnahmen zum Arbeitsumfeld seitens des Arbeitsgebers zu nutzen, da die vorgeschlagenen Angebote in den meisten Fällen nicht vorhanden waren. Lediglich an Angeboten zur Karriereplanung konnte die Mehrzahl teilnehmen, der Großteil empfand dies als hilfreich. In den Freitextantworten wurde vor allem ein rücksichtsvollerer Umgang in der Familien- und Erziehungsorganisation gefordert, sodass ein Nebeneinander von Job und Familie ermöglicht werde. Außerdem wurde auf die Notwendigkeit eines Dienstsystems hingewiesen, welches ein pünktliches Arbeitsende und eine flexiblere, individuellere Gestaltung zulässt. Nebendies wurde mehr Geschlechtergleichheit im Arbeitsalltag gefordert, sodass für beide Geschlechter u. a. ein Netzwerkausbau erleichtert werden könnte.

\subsection{Zufriedenheit der Teilnehmenden}

\subsubsection{Signifikante Unterschiede zwischen besonders zufriedenen und zufriedenen Teilnehmenden}

Um herauszuarbeiten, was die besonders zufriedenen von den zufriedenen Teilnehmenden unterscheidet, wurde aus allen Teilnehmenden $(\mathrm{N}=132)$ zwei Gruppen gebildet. Die erste Gruppe der beruflich besonders zufriedenen Chirurginnen und Chirurgen beinhaltete die Teilnehmenden, die eine hohe subjektive berufliche Zufriedenheit von mindestens $90 \%$ angaben ( $n=39$ ), in der zweiten Gruppe befanden sich die restlichen Teilnehmenden $(n=93)$. In Tabelle 6 sind allein die signifikanten Unterschiede zwischen den genannten Gruppen aufgeführt.

Tabelle 6: Unterschiede zwischen den beruflich besonders zufriedenen Teilnehmenden und den beruflich zufriedenen Teilnehmenden

$\mathrm{N}=132$, nicht alle Items wurden von allen Teilnehmenden beantwortet, die $\mathrm{p}$-Werte beziehen sich auf den Unterschied zwischen den besonders zufriedenen Teilnehmenden und den zufriedenen Teilnehmenden.

\begin{tabular}{|c|c|c|c|c|}
\hline Items & $\begin{array}{l}\text { zufrieden }(10-80 \%) \\
n=93\end{array}$ & $\begin{array}{l}\text { besonders } \\
\text { zufrieden } \\
(90-100 \%) \\
n=39\end{array}$ & $\begin{array}{l}\text { signifikante } \\
\text { Werte }\end{array}$ & p- \\
\hline \multicolumn{5}{|l|}{ Alter [Mittelwert in Jahren] } \\
\hline $\begin{array}{l}\text { zum Zeitpunkt der } \\
\text { Umfrage }\end{array}$ & 45,8 & 49,8 &, $006^{b}$ & \\
\hline bei Habilitation & 39,1 & 37,8 &, $043^{b}$ & \\
\hline
\end{tabular}




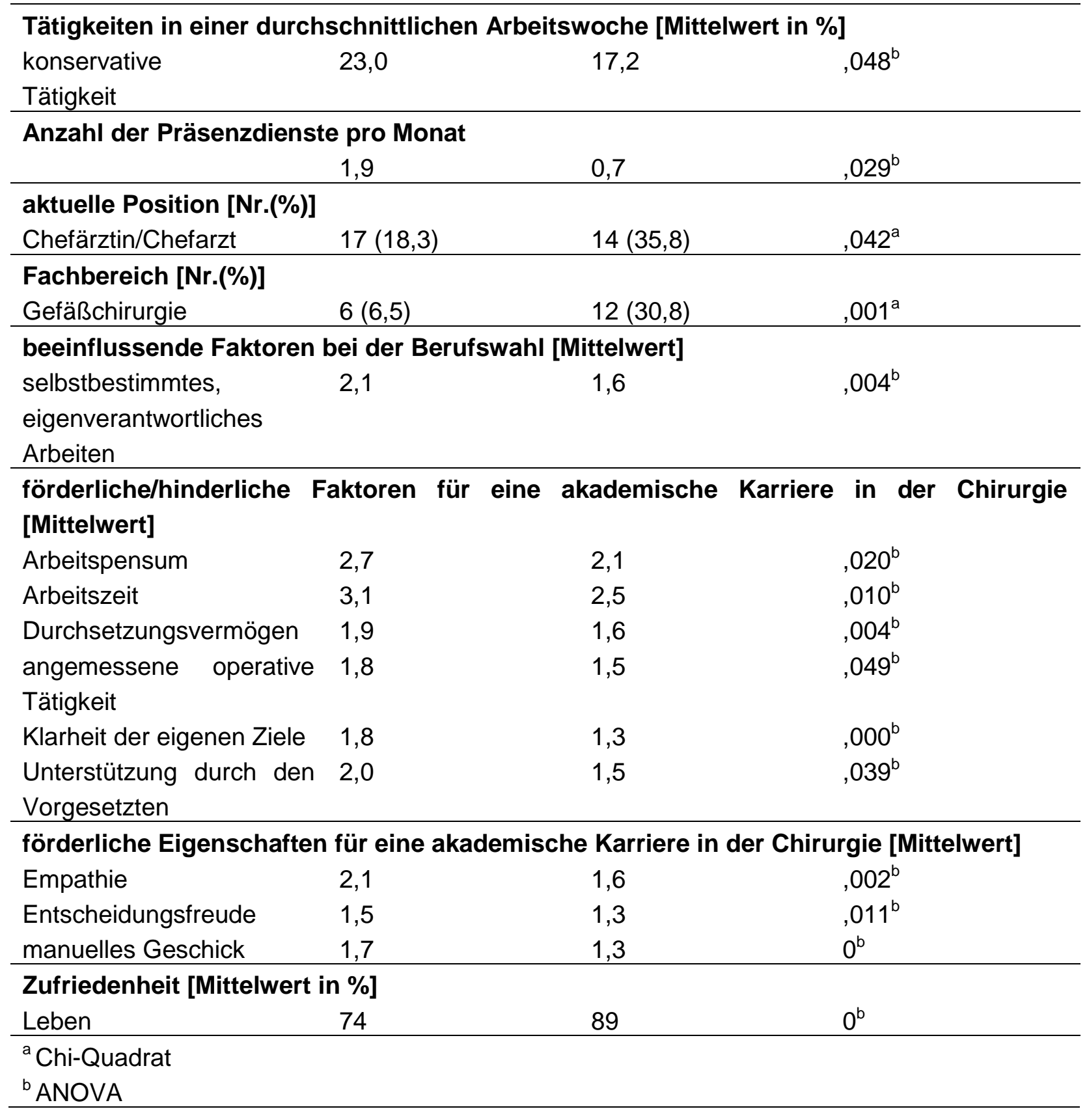

Im Durchschnitt waren die besonders zufriedenen Teilnehmenden vier Jahre älter $(49,8$ versus $45,8, p<, 05) .45 \%(n=14)$ der Teilnehmenden, die eine chefärztliche Position innehaben, empfanden sich als beruflich besonders zufrieden. Diese besonders zufriedenen Teilnehmenden investierten in einer durchschnittlichen Arbeitswoche weniger Arbeitszeit in konservative Tätigkeiten und absolvierten weniger Präsenzdienste pro Monat $(p<, 05)$. Außerdem erachteten die besonders zufriedenen Teilnehmenden die Arbeitszeit und das Arbeitspensum als weniger hinderlicher für den beruflichen Aufstieg. 
Die besonders zufriedenen Chirurginnen und Chirurgen bewerteten „Durchsetzungsvermögen“, eine „angemessene operative Tätigkeit“, „Klarheit der eigenen Ziele“ sowie "Unterstützung durch die Vorgesetzten durchschnittlich als förderlicher für den beruflichen Aufstieg $(p<, 05)$.

In Bezug auf förderliche Eigenschaften für die Karriere unterschieden sich die besonders zufriedenen und die zufriedenen Teilnehmenden in "Empathie“, „Entscheidungsfreude“ und „manuellem Geschick“ voneinander. Diese Eigenschaften bewerteten die besonders zufriedenen Teilnehmenden durchschnittlich als wichtiger für den beruflichen Aufstieg $(p<, 05)$.

Zusammenfassend betrachtet, waren die besonders zufriedenen Teilnehmenden vier Jahre älter zum Zeitpunkt der Umfrage und ein Jahr jünger bei Abschluss der Habilitation. Sie befanden sich in einer fortgeschrittenen beruflichen Position und profitierten mehr von einem selbstbestimmten, eigenverantwortlichen Arbeiten. Sie absolvierten weniger Dienste pro Monat und fühlten sich durch das Arbeitspensum und die Arbeitsdienste weniger beeinträchtigt als die Vergleichsgruppe.

\subsubsection{Prädiktoren für eine hohe berufliche Zufriedenheit}

Eine Regressionsanalyse sollte prüfen, ob eine hohe berufliche Zufriedenheit (abhängige Variable) durch Prädiktoren (unabhängige Variable) beeinflusst wird.

Es wurden hierfür aus den besonders zufriedenen Teilnehmenden geschlechtsabhängig zwei Subgruppen gebildet (vergleiche vorheriges Kapitel). Hieraus ergaben sich 17 (27,4\%) Frauen und 22 (31,4\%) Männer, die eine über neunzigprozentige Zufriedenheit angaben.

Tabelle 7 zeigt eine Übersicht der relevanten Prädiktoren, welche einen Einfluss auf die hohe berufliche Zufriedenheit nahmen. Dargestellt werden die Werte der Regressionsanalyse durch den Beta-Koeffizienten (B). Außerdem befinden sich in der rechten Spalte die Signifikanzen nach Anwendung einer ANOVA, die Unterschiede zwischen den beruflich besonders zufriedenen Frauen und Männern maß. 
Tabelle 7: Prädiktoren einer hohen beruflichen Zufriedenheit

Regressionsanalyse mit der beruflichen Zufriedenheit (>90\%) als abhängige Variable

$\mathrm{N}=39$, nicht alle Items wurden von allen Teilnehmende beantwortet, dargestellt ist der deskriptive Mittelwert und der BetaKoeffizient, die p-Werte beziehen sich auf die Unterschiede zwischen besonders zufriedenen Frauen und den besonders zufriedenen Männern

\begin{tabular}{|c|c|c|c|c|c|}
\hline \multirow[b]{2}{*}{$\begin{array}{l}\text { Unabhängige } \\
\text { Variable }\end{array}$} & \multicolumn{2}{|c|}{ Frauen $n=17(27.4 \%)$} & \multicolumn{2}{|c|}{ Männer n= 22 (31.4\%) } & \multirow[t]{2}{*}{ p-Wert ${ }^{a}$} \\
\hline & $\begin{array}{l}\text { deskriptive } \\
\text { Statistik } \\
\text { [Mittelwert] }\end{array}$ & $\begin{array}{l}\text { standardi } \\
\text { sierter B- } \\
\text { Koeffizien } \\
t\end{array}$ & $\begin{array}{l}\text { deskriptive } \\
\text { Statistik } \\
\text { [Mittelwert] }\end{array}$ & $\begin{array}{l}\text { standardisi } \\
\text { erter B- } \\
\text { Koeffizient }\end{array}$ & \\
\hline \multicolumn{6}{|l|}{ Kinderbetreuung } \\
\hline durch mich selbst & $\begin{array}{l}21,0 \\
\% / \text { Woche }\end{array}$ & $-1,04^{b}$ & $\begin{array}{l}8,4 \\
\% / \text { Woche }\end{array}$ &, 12 & ,001 \\
\hline $\begin{array}{l}\text { durch Partnerin und } \\
\text { Partner }\end{array}$ & $\begin{array}{l}22,9 \\
\% / \text { Woche }\end{array}$ &,$- 24^{b}$ & $\begin{array}{l}50,7 \\
\% / \text { Woche }\end{array}$ &,- 38 & ,019 \\
\hline $\begin{array}{lr}\text { durch } & \text { eine } \\
\text { Betreuung } & \text { im } \\
\text { Haushalt } & \end{array}$ & $\begin{array}{l}33,0 \\
\% / \text { Woche }\end{array}$ &,$- 83^{b}$ & $\begin{array}{l}6,8 \\
\% / \text { Woche }\end{array}$ & $\begin{array}{l}\text { Nicht } \\
\text { darstellbar }\end{array}$ &, 001 \\
\hline $\begin{array}{l}\text { durch } \\
\text { Schule/Hort/Kinderg } \\
\text { arten }\end{array}$ & $\begin{array}{l}25,5 \\
\% / \text { Woche }\end{array}$ & $-1,33^{b}$ & $\begin{array}{l}28,3 \\
\% / \text { Woche }\end{array}$ &,- 18 &, 749 \\
\hline \multicolumn{6}{|l|}{$\begin{array}{l}\text { Berufstätigkeit } \\
\text { des Partners }\end{array}$} \\
\hline & $\begin{array}{l}54,6 \\
\text { Std./Woche }\end{array}$ & ,08 & $\begin{array}{l}14,0 \\
\text { Std/Woche }\end{array}$ &,$- 29^{b}$ &, 001 \\
\hline \multicolumn{6}{|l|}{$\begin{array}{l}\text { förderliche } \\
\text { Faktoren }\end{array}$} \\
\hline $\begin{array}{l}\text { Unterstützung durch } \\
\text { den Vorgesetzten }\end{array}$ & 1,29 &,$- 41^{b}$ & 1,77 &,- 03 &, 105 \\
\hline \multicolumn{6}{|l|}{$\begin{array}{l}\text { förderliche } \\
\text { Eigenschaften }\end{array}$} \\
\hline manuelles Geschick & 1,29 &,$- 41^{b}$ & 1,32 &,- 06 &, 516 \\
\hline
\end{tabular}

${ }^{a}$ ANOVA zwischen den besonders zufriedenen Frauen und den besonders zufriedenen Männern $(90 \%-100 \%)$, signifikante Werte $(p<, 05)$ sind in „fett" dargestellt

brelevante Werte (B-Koeffizient $>0,4$ ) sind in „kursiv“ dargestellt

${ }^{c}$ nicht alle Teilnehmenden haben das Item markiert 
Bei Beleuchtung der Betreuungssituation der Kinder fiel auf, dass jegliche Art der Kinderbetreuung einen negativen Einfluss auf die berufliche Zufriedenheit der Chirurginnen hatte $(B=-83-B=1,33)$, bei den Chirurgen waren keine relevanten Prädiktoren im Bereich der Kinderbetreuung feststellbar. Bei diesen übte hingegen die „Arbeitszeit des Partners/Partnerin“ einen negativen Effekt auf die Zufriedenheit aus, $(B=-, 29)$.

Das Item „Unterstützung durch den Vorgesetzten“ konnte als positiver Prädiktor für die Zufriedenheit der Frauen identifiziert werden, $(B=-, 41)$. Die Polung (eins=förderlich/fünf=hinderlich) des Items kehrte das negative Vorzeichen des BetaKoeffizienten ins Gegenteil, sodass eine förderliche Unterstützung mit einer höheren Zufriedenheit einhergeht. Dieses ist ebenfalls bei dem Item "manuelles Geschick“ der Fall, $(B=-, 41)$.

Verglich man die Gruppen der besonders zufriedenen Frauen mit den besonders zufriedenen Männern, fielen signifikante Unterschiede im Bereich der Kinderbetreuung auf $(p<, 05)$. Außerdem konnte ein weiterer Unterschied in den Angaben zur Arbeitszeit der Partnerin und des Partners gezeigt werden. Die Partnerin oder der Partner der besonders zufriedenen Männer arbeiteten im Schnitt weniger als die Partnerin oder der Partner der besonders zufriedenen Frauen $(p<, 05)$.

Zusammenfassend übten sowohl alle Variablen der Kinderbetreuung als auch die Arbeitszeit der Partnerin oder des Partners einen negativen Effekt auf die berufliche Zufriedenheit der Chirurginnen und der Chirurgen aus. Ein positiver Effekt kann bei „Unterstützung durch Vorgesetzte“ und „manuellem Geschick“ angenommen werden. Je mehr Gewichtung die Teilnehmenden diesen Items beimaßen, desto höher war die berufliche Zufriedenheit.

\subsection{Einfluss von Elternschaft auf eine chirurgische Karriere}

\subsubsection{Signifikante Unterschiede zwischen kinderlosen Teilnehmenden und} Teilnehmenden mit Kind

Um Unterschiede zwischen den Teilnehmenden mit Kind und den kinderlosen Teilnehmenden herauszuheben, wurden Signifikanztests zwischen diesen Gruppen 
durchgeführt (Teilnehmende mit Kind vs. Teilnehmende ohne Kind). Tabelle 8 zeigt die signifikanten Ergebnisse zwischen den Gruppen.

Tabelle 8: Signifikante Unterschiede zwischen kinderlosen Teilnehmenden und Teilnehmenden mit Kind

$\mathrm{N}=120$, nicht alle Items wurden von allen Teilnehmenden beantwortet, die $\mathrm{p}$-Werte beziehen sich auf die Unterschiede zwischen den kinderlosen Teilnehmenden und den Teilnehmenden mit Kind

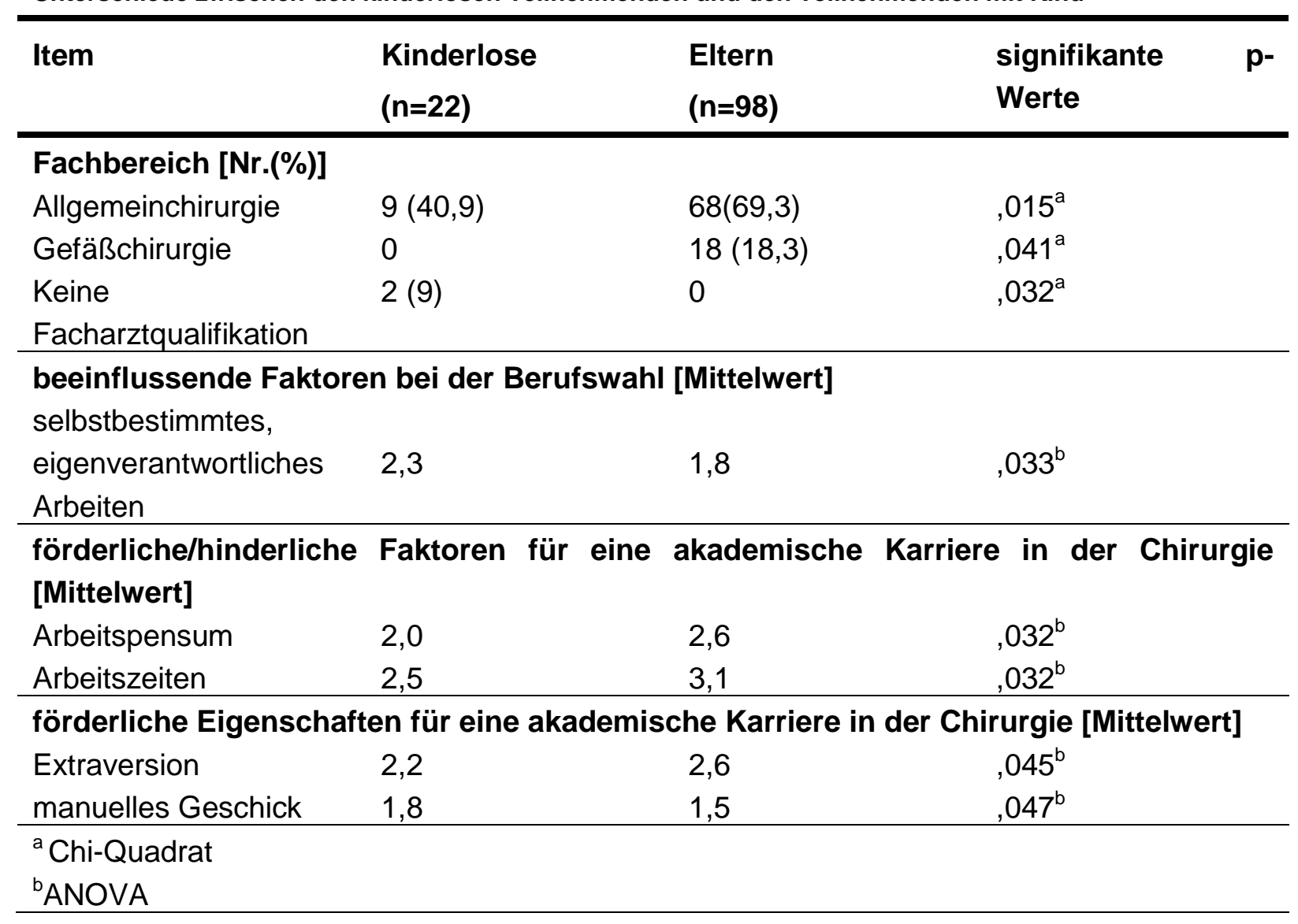

Bei Beurteilung der förderlichen und hinderlichen Faktoren empfanden die Teilnehmenden mit Kind einerseits das „Arbeitspensum“ und andererseits die „Arbeitszeiten“ als hinderlicher für den beruflichen Aufstieg $(p<, 05)$.

„Extraversion“ und „manuelles Geschick“ wurden sowohl von den Eltern als auch von den kinderlosen Teilnehmenden als wichtige Eigenschaften für den beruflichen Erfolg bewertet, jedoch empfanden die Eltern „Extraversion“, die kinderlosen Teilnehmenden hingegen das „manuelle Geschick“ als weniger wichtig $(p<, 05)$.

Bei der Wahl des Berufes spielte bei beiden Gruppen das „eigenverantwortliche und selbstbestimmte Arbeiten“ eine Rolle, die Teilnehmenden mit Kind schätzen dies als noch wichtiger ein $(p<, 05)$. 
Zusammenfassend fühlten sich die Teilnehmenden mit Kind durch Arbeitszeiten und Arbeitsumfang stärker eingeschränkt als die kinderlosen Teilnehmenden. Ein selbstbewusstes, selbstbestimmtes Agieren und ein selbstorganisiertes Arbeitsumfeld spielte bei den Chirurginnen und Chirurgen mit Kind eine größere Rolle als bei den Teilnehmenden ohne Kind, wobei auch diese den Items insgesamt eine große Relevanz beimaßen.

\subsubsection{Signifikante Unterschiede zwischen Müttern und Vätern}

Um festzustellen, ob Unterschiede zwischen den Müttern und Vätern unter den Teilnehmenden existierten, wurden Signifikanztests durchgeführt (Mütter versus Väter).Tabelle 9 zeigt die jeweils signifikanten Ergebnisse beider Gruppen.

Tabelle 9: Signifikante Unterschiede zwischen Müttern und Vätern

$\mathrm{N}=98$, nicht alle Items wurden von allen Teilnehmenden beantwortet, die $\mathrm{p}$-Werte beziehen sich auf die Unterschiede zwischen den Müttern und Vätern

\begin{tabular}{|c|c|c|c|c|}
\hline Item & Mütter ( $n=48)$ & Väter $(n=50)$ & $\begin{array}{l}\text { signifikante } \\
\text { Werte }\end{array}$ & p- \\
\hline \multicolumn{5}{|l|}{ Alter [Mittelwert in Jahren] } \\
\hline bei Promotion & 27,5 & 28,8 &, $006^{\mathrm{b}}$ & \\
\hline bei Geburt erstes Kind & 36 & 32,8 &, $004^{\mathrm{b}}$ & \\
\hline \multicolumn{5}{|l|}{ aktuelle Position [Nr.(\%)] } \\
\hline Facharzt/ärztin & $5(10.4)$ & 0 &, $025^{\mathrm{a}}$ & \\
\hline \multicolumn{5}{|c|}{ berufliche Position während der Geburt des ersten Kindes [Nr.(\%)] } \\
\hline $\begin{array}{l}\text { während der fachärztlichen } \\
\text { Weiterbildung }\end{array}$ & $3(6.25)$ & $26(52)$ &, $000^{a}$ & \\
\hline \multicolumn{5}{|c|}{ Tätigkeiten in einer durchschnittlichen Arbeitswoche [Mittelwert in \%] } \\
\hline konservative Tätigkeit & 25,5 & 18,4 &, $034^{\mathrm{b}}$ & \\
\hline Lehre & 12,1 & 7,9 &, $000^{\mathrm{b}}$ & \\
\hline \multicolumn{5}{|c|}{ tatsächliche Arbeitszeit pro Woche [Mittelwert in Stunden] } \\
\hline & 59,8 & 65 &, $033^{\mathrm{b}}$ & \\
\hline \multicolumn{5}{|c|}{ Pause nach Geburt in Monaten [Mittelwert] } \\
\hline & 5,2 & 0,16 &, $000^{\mathrm{b}}$ & \\
\hline \multicolumn{5}{|c|}{ Kinderbetreuung in einer durchschnittlichen Arbeitswoche [Mittelwert in \%] } \\
\hline selbst & 27,6 & 7.2 &, $002^{\mathrm{b}}$ & \\
\hline Partner & 15,8 & 58,2 &, $000^{\mathrm{b}}$ & \\
\hline Betreuung im Haushalt & 21,7 & 4,0 &, $002^{b}$ & \\
\hline KITA/Schule/Hort & 43,8 & 30,4 &, $026^{\mathrm{b}}$ & \\
\hline \multicolumn{5}{|c|}{ Beeinflussende Faktoren bei der Berufswahl [Mittelwert] } \\
\hline Karriere & 3,4 & 2,8 &, $007^{\mathrm{b}}$ & \\
\hline
\end{tabular}




\begin{tabular}{|c|c|c|c|c|}
\hline Verdienst & 3,6 & 3,1 &, $001^{b}$ & \\
\hline $\begin{array}{l}\text { förderliche/hinderliche Fakt } \\
\text { [Mittelwert] }\end{array}$ & ren $\mathrm{fi}$ & lemisc & in der & Chirurgie \\
\hline Geschlecht & 3,6 & 2,5 &, $000^{b}$ & \\
\hline Familienstand & & &, $004^{a}$ & \\
\hline ledig & 8 & 0 & & \\
\hline verheiratet/in Partnerschaft & 34 & 47 & & \\
\hline getrennt/geschieden/verwitwet & 6 & 3 & & \\
\hline Arbeitszeit des Partners in S & unden & Mittelu & & \\
\hline & 52,9 & 19,8 &, $000^{\mathrm{b}}$ & \\
\hline Zeiteinteilung in einer durchs & chnittli & [Mitte & & \\
\hline Haushalt & 8,02 & 5,44 &, $023^{b}$ & \\
\hline förderliche Eigenschaften für & eine a & Karriel & urgie [Mit & telwert] \\
\hline Anpassungsfähigkeit & 2,1 & 1,6 &, $005^{\mathrm{b}}$ & \\
\hline gute Selbstorganisation & 1,2 & 1,5 &, $030^{\mathrm{b}}$ & \\
\hline $\begin{array}{l}\text { a Chi-Quadrat } \\
\text { b ANOVA }\end{array}$ & & & & \\
\hline
\end{tabular}

Die Mütter $(n=48)$ unter den Teilnehmenden erlangten die Promotion im Durchschnitt etwa ein Jahr früher als die Väter $(n=50)(p<, 05)$. Das erste Kind bekamen sie hingegen drei Jahre später als die Männer, mit 36 Jahren $(p<, 05)$. Drei Frauen befanden sich bei der Geburt des ersten Kindes in der fachärztlichen Weiterbildung $(\mathrm{p}<, 05)$.

Die Betreuung der Kinder übernahmen die Mütter selbst oder eine Betreuung im Haushalt, 15,8\% wurde an den Partner oder die Partnerin übergeben. Bei den Vätern wurden knapp $60 \%$ von diesen übernommen $(p<, 05)$. Eine Betreuung durch eine Kindertagesstätte/Hort/Schule fand bei den Müttern zu 43,8\%, bei den Vätern zu $30 \%$ statt $(p<, 05)$. Unter den Vätern war kein Teilnehmer alleinstehend, bei den Müttern waren es acht. Die Partner oder Partnerin der Mütter arbeiteten pro Woche mehr als die der Väter $(p<, 05)$. Ebenso investierten die Mütter in einer durchschnittlichen Woche mehr Freizeit in Haushaltsarbeit als die Väter $(\mathrm{p}<, 05)$.

Die Väter hingegen arbeiteten in einer Arbeitswoche etwa fünf Stunden mehr als die Mütter $(p<, 05)$. Während dieser Arbeitswoche verbrachten die Väter allerdings im Schnitt weniger Zeit mit konservativen Tätigkeiten und Lehre als die Mütter $(p<, 05)$. Die Motive „Karriere“ und „Verdienst“ empfanden die Mütter als unwichtiger für die Berufswahl $(p<, 05)$. Eine „gute Selbstorganisation“ als wichtige Eigenschaft für den 
beruflichen Aufstieg schätzten die Mütter als relevanter für den beruflichen Aufstieg ein, die Männer hingegen empfanden „Anpassungsfähigkeit“ wichtiger $(p<, 05)$.

Zusammenfassend erlangten die Mütter unter den Teilnehmenden früher die Promotion, bekamen jedoch später ihr erstes Kind als die Väter. Der Großteil der Kinderbetreuung wurde bei den Müttern durch eine externe Einrichtung, bei den Vätern durch die Partnerin oder den Partner übernommen. Die Väter verbrachten pro Arbeitswoche etwa fünf Stunden länger bei der Arbeit, wohingegen die Mütter während ihrer Arbeitszeit mehr Zeit in die konservative Tätigkeit und Lehre investierten. 


\section{Diskussion}

Die vorliegende Studie erhob biographische Merkmale und Einschätzungen zu Karrierefaktoren, die habilitierte Chirurginnen und Chirurgen in Deutschland in einer Online-Umfrage wiedergaben. Es konnten eine Reihe von Persönlichkeitsmerkmalen und Faktoren identifiziert werden, die eine Karriere in der akademischen Chirurgie an einem fortgeschrittenen Punkt ausmachen und als förderlich von den Befragten empfunden wurden. Die Ergebnisse können als ein Fallbeispiel für weitere akademische Fachbereiche herangezogen werden, da in vielen Fällen Frauen in Führungspositionen eine Rarität darstellen und noch immer allzu häufig ein deutliches Geschlechtergefälle in akademischen Berufen festgestellt werden kann. Nichtsdestoweniger sollte keinesfalls auf die Gesamtheit geschlossen werden, da die vorliegende Arbeit selbstverständlich nur einen Ausschnitt aller Charakteristika der deutschen habilitierten Chirurginnen und Chirurgen darstellt.

\subsection{Auswahl der Stichprobe}

Es wurden bewusst diejenigen Ärztinnen und Ärzte ausgewählt, die bereits eine erfolgreiche Karriere in einem ausgewählten Fachgebiet (hier der Chirurgie) vorweisen konnten. "Erfolg“ wird in diesem Zusammenhang definiert als eine abgeschlossene Habilitation, die berufliche Position oder die Institution, an der die Teilnehmenden beschäftigt waren, stellten kein Einschlusskriterium für die Studie dar.

Zu Beginn der Studie war die Überlegung, auch die Wissenschaftlerinnen und Wissenschaftler zu rekrutieren, die sich gegen eine akademische Laufbahn entschieden haben. Um allerdings nicht bereits bei der Auswahl und Einladungen der Teilnehmenden einen negativen Eindruck bei denjenigen zu erwecken, die sich aus unterschiedlichsten Gründen gegen eine akademische Laufbahn entschieden haben, wurde auf diese Methode verzichtet. Die Ermittlung der Adressen derjenigen, die eine akademische Laufbahn abgebrochen haben, wäre ohnehin problematisch gewesen.

In diesem Zusammenhang muss auf die Arbeit von Levine et al. hingewiesen werden, die Interviews mit weiblichen Medizinerinnen durchführten, um von den 
Gründen eines Abbruchs zu erfahren (Levine et al. 2011). Außerdem sollte die britische Umfrage unter chirurgischen Assistenzärztinnen und Assistenzärzten von Richards et. al. erwähnt werden, die Einflussfaktoren auf Karriereentscheidungen identifizierte (Richards et al. 2009). Daneben hat die Klinik für Allgemeinmedizin des Universitätskrankenhauses Hamburg das Projekt "TransFerGenderMed“ mit dem Ziel konzeptioniert, förderliche und hemmende Bedingungen während des Karriereverlaufs zu identifizieren (Uke.de - Institut und Poliklinik für Allgemeinmedizin - Projekt Transfergendermed 2017). Der Fokus dieser Studien lag jedoch in den Anfängen und im speziellen während der Weiterbildungszeit einer Karriere und nahm kaum Bezug auf den spezifischen akademischen Weg.

\subsection{Wahl der Weiterbildungsdisziplin Chirurgie}

Um herauszuarbeiten, inwieweit das Geschlecht Einfluss auf die Entscheidung des Fachgebiets nimmt, wurden die Teilnehmenden nach den Motiven ihrer Berufswahl befragt. Es wurde angenommen, dass Frauen und Männer durch unterschiedliche Motive geleitet werden, eine Karriere in der Chirurgie einzugehen.

„Interesse/Fähigkeit/Eignung" nannten sowohl die Chirurginnen als auch die Chirurgen als den wichtigsten Grund für Ihre Berufswahl. Nachfolgend wurden „eigenverantwortliches Arbeiten“ und „Entfaltungsmöglichkeiten“ genannt. Einflüsse, die eine selbstständige, weitestgehend unabhängige und verantwortungsvolle Arbeit ermöglichen. Diese Aspekte decken sich mit der Arbeit von Weber et. al., die auf Grundlage einer Umfrage unter operativ tätigen Chirurginnen an Universitätskliniken außerdem die rasche und effektive Heilung des Patienten hervorhoben (Weber et al. 2005). Wendel et al. unterstrichen in ihrer Arbeit den hohen intellektuellen Anspruch während chirurgischer Arbeit, was weitere Literatur bestätigt (Wendel et al. 2003; Weber et al. 2005; Wyrzykowski et al. 2006).

Im Gegensatz dazu argumentierten Williams und Cantillon, dass die praktische Arbeit einen immer geringeren Stellenwert einnimmt, weil angehende Chirurginnen und Chirurgen meist die unbeliebte Stationsarbeit erledigen müssen und seltener operativ tätig sind (Williams und Cantillon 2000). Bei den Teilnehmerinnen der Umfrage von Williams und Cantillon handelte es sich jedoch um Frauen in den Anfängen der chirurgischen Weiterbildung, und Teile der Stationsarbeit können in der 
Regel mit Fortschritt der Karriere abgegeben werden. Generell betrachtet ist eine selbstständige Gestaltung des Arbeitsumfeldes häufig an eine höhere hierarchische Position gekoppelt und stellt bei Frauen und Männern einen der Gründe dar, weshalb eine Führungsposition angestrebt wird (Mayr 2008). Auch bei den Teilnehmenden der vorliegenden Umfrage wurde, wie bereits erwähnt, das eigenverantwortliche Arbeiten als einer der wichtigsten Gründe der Berufswahl genannt.

Die Aufstiegschancen in der Chirurgie scheinen zunächst erkennbar und erreichbar zu sein, sodass sich viele Studierende von der chirurgischen Fachdisziplin angezogen fühlen (Azizzadeh et al. 2003; Sanfey et al. 2006; Sutton et al. 2014; Baschera et al. 2015). Bei den Teilnehmenden der vorliegenden Umfrage schienen Motive wie "Karriere" und „Verdienst" nur bedingt einen relevanten Einfluss auf die Wahl der Fachrichtung Chirurgie zu nehmen, im Geschlechtervergleich maßen die Chirurgen diesen Motiven mehr Gewichtung bei. Dieses deckt sich mit weiterer Literatur, in der Männer generell betrachtet, stärker durch Einkommen, Prestige und potentielle Aufstiegschancen bei der Berufswahl beeinflusst werden (Baxter et al. 1996; Ewi-psy.fu-berlin.de - Unterschiedliche Berufsverläufe, Berufserfolg und Lebensbedingungen von Frauen und Männern in zwei hochqualifizierten Berufen Forschungsbericht Nr. 16 2001; Sanfey et al. 2006; Saunders et al. 2008; Baschera et al. 2015). In diesem Kontext muss auch die Studie zur Professionalisierung und Integration von Lebenssphären (PROFIL) der FU Berlin genannt werden, aus der hervorgeht, dass Frauen eher in randständigen teils außeruniversitären Fachgebieten eine Führungsposition einnehmen (Forschung, Verwaltung, Management) (Ewi-psy.fu-berlin.de - Fragebogen und Interviewleitfaden im Projekt "PROFIL“ (Professionalisierung und Integration der Lebenssphären. Geschlechtsspezifische Berufsverläufe in Medizin und Psychologie) Forschungsbericht Nr. 14 1999; Mayr 2008). Die Männer stiegen dagegen eher in etablierten, zentralen Fachgebieten auf, darunter auch jene, denen das größte Prestige (u. a. Chirurgie) zugesprochen wird (Ewi-psy.fu-berlin.de - Fragebogen und Interviewleitfaden im Projekt „PROFIL“ (Professionalisierung und Integration der Lebenssphären. Geschlechtsspezifische Berufsverläufe in Medizin und Psychologie) - Forschungsbericht Nr. 14 1999). Dieses kann durch die vorliegenden Ergebnisse bekräftigt werden, nach denen die teilnehmenden Frauen mehr Arbeitszeit in konservative Arbeit und Lehre investierten. Die Folge der Randgruppenbildung ist, 
dass vor allem Frauen die Chirurgie aus Mangel an weiblichen Vorbildern in höheren Positionen nicht in Betracht ziehen (Baxter et al. 1996; Williams und Cantillon 2000; Neumayer et al. 2002; Sanfey et al. 2006; Cochran et al. 2013; Hill und Vaughan 2013). In der vorliegenden Studie wurde der "Einfluss durch Dritte" allerdings von den Teilnehmenden in das letzte Drittel aller Motive eingegliedert und für die männlichen Chirurgen nahm es einen größeren Stellenwert ein, als für die teilnehmenden Frauen. Ein signifikanter Unterschied zwischen den Geschlechtern ließ sich jedoch nicht feststellen.

Als ein weiterer eher unwichtiger Grund wurde die „Vereinbarkeit von Privatleben und Beruf" gewertet. Die Überschneidung der langen Ausbildungszeit und der Jahre, in denen eine Familie gegründet wird, scheint noch immer ein Problem dazustellen und Frauen und Männer von der Chirurgie abzuschrecken (Baxter et al. 1996; Williams und Cantillon 2000; Azizzadeh et al. 2003; Wendel et al. 2003; Sanfey et al. 2006; Richards et al. 2009; Sutton et al. 2014). Berufsentscheidungen beruhen gerade im chirurgischen Feld nicht nur auf Karriereaussichten, sondern vielmehr auf der Aussicht eines attraktiven Privatlebens (Sanfey et al. 2006; McCord et al. 2007; Stabile 2008). Das Wissen, dass Studierende und Ärztinnen und Ärzte, die eine chirurgische Laufbahn einschlagen, mehr Arbeitszeiten und -schichten in Kauf nehmen müssen als diejenigen, die eine andere Fachrichtung wählen (Frank et al. 1998; Baschera et al. 2015), dürfte die Entscheidung für die Chirurgie nicht vereinfachen.

Die vollen Fähigkeiten der sogenannten "Generation Y“ können nur dann ausgeschöpft werden, wenn es ein Umdenken durch die Arbeitgeber gebe (Schmidt et al. 2011). Die Generation Y, das sind die zwischen 1977 und 1994 Geborenen, fordert eine besondere Verzahnung von Privatleben und Beruf (Schmidt et al. 2012) und stellt Verhältnisse und Vorstellungen in Frage, die bisher als selbstverständlich galten (Parment 2011). Während die Studierenden 1980 einen ausgewogenen Lifestyle als nicht so entscheidend für ihre Berufswahl (Tardiff et al. 1986) ansahen, nimmt die aktuelle "Generation Y" zusehends Abstand von der alleinigen Identifikation durch den Job (Saunders et al. 2008). Dies macht den Umgang mit Vorgesetzten schwer, da Hierarchien abgelehnt werden und gleichzeitig eine gute und eng geführte Ausbildung durch Lehrende gefordert werde (Schmidt et al. 2011; Schmidt et al. 2012). Kampagnen für familienfreundliche Krankenhäuser wie 
beispielweise durch den Marburger Bund (Marburger Bund - Für ein familienfreundliches Krankenhaus 2014) machen es sich zur Aufgabe, den Trend einer ausgeglichenen Work-Life-Balance zu unterstützen.

Die Annahme, dass es Geschlechtsunterschiede bei der Auswahl der Fachdisziplin gebe, konnte nur bedingt bestätigt werden. Es konnten zwar einzelne signifikante Unterschiede bei der Begründung der Berufswahl identifiziert werden, generell strebten habilitierte Chirurginnen und Chirurgen aber aus ähnlichen Gründen eine Karriere in der Chirurgie an (Vergleiche Hypothese fünf, Seite 8). In diesem Zusammenhang muss allerdings explizit darauf hingewiesen werden, dass es sich bei der vorliegenden Stichprobe bereits um eine selektionierte Grundgesamtheit handelte und Frauen und Männer am fortgeschrittenen Karrierepunkt der Habilitation möglicherweise sehr ähnliche Anreize der Berufswahl äußerten. Ohne eine Selektion im Vorfeld wären die Unterschiede womöglich signifikanter.

\subsection{Karriereentwicklung}

\subsubsection{Persönlichkeitsstruktur in der Chirurgie}

Vor dem Hintergrund, dass nicht-kognitive Eigenschaften eine medizinische Laufbahn mindestens so stark beeinflussen wie kognitive Fähigkeiten (Schwartz et al. 1994), wurden auch Erstgenanntes rückblickend im Hinblick auf die Karriere eingeschätzt. Es wurde angenommen, dass es bestimmte Faktoren gäbe, die eine Karriere in der Chirurgie positiv oder negativ beeinflussen. Zusätzlich war die Überlegung, dass sich diese Karrierefaktoren zwischen den Geschlechtern unterscheiden.

Die Chirurginnen schätzten die mit Selbstdisziplin assoziierten Attribute wie "Gewissenhaftigkeit" und eine „gute Selbstorganisation" als wichtiger für die Karriere ein als ihre männlichen Kollegen. Darüber hinaus muss in diesem Zusammenhang die "Risikobereitschaft“ genannt werden, der die Frauen ebenso mehr Gewichtung beimaßen als die Männer.

Die Literatur bezüglich nützlicher chirurgischer Merkmale und Eigenschaften ist abhängig von der Art der Studie sehr heterogen. Es ist jedoch etabliert, dass "Gewissenhaftigkeit" unter Chirurginnen und Chirurgen eine sehr große Bedeutung 
zugeschrieben wird (McGreevy und Wiebe 2002; Hoffman et al. 2010; Drosdeck et al. 2015). Ein Vergleich zwischen Chirurginnen und Chirurgen und Ärztinnen und Ärzten aus anderen Fachdisziplinen zeigte, dass Chirurginnen und Chirurgen "Gewissenhaftigkeit" und „Extraversion" als signifikant wichtiger (Hoffman et al. 2010; Drosdeck et al. 2015), "Verträglichkeit“ hingegen als unwichtiger bewerteten (Drosdeck et al. 2015). Durch einen Subgruppenvergleich zwischen beruflich fortgeschrittenen Chirurginnen und Chirurgen und chirurgischen Assistenzärztinnen und -ärzten konnte gezeigt werden, dass "Gewissenhaftigkeit“ und „Extraversion“ von den beruflich fortgeschrittenen Teilenehmenden als wichtiger empfunden wurde, „Verträglichkeit" wurde hingegen von den Assistenzärztinnen und Assistenzärzten als hilfreicher bewertet (Drosdeck et al. 2015). Dies könnte mit dem hierarchischen Erleben im Berufsalltag erklärbar sein, welches auf der unteren Karrierestufe häufig präsenter ist.

Ein Vergleich zwischen der Persönlichkeit von Chirurginnen und Chirurgen und der Allgemeinheit durch McGreevy und Wiebe zeigte Parallelen zu vorgenannten Ergebnissen. Darüber hinaus maßen die Chirurginnen hier auch im Vergleich zu ihren männlichen Kollegen den Attributen wie Selbstdisziplin, Durchhaltevermögen, Fleiß und Ehrgeiz mehr Gewichtung bei (McGreevy und Wiebe 2002). Diese Ergebnisse befinden sich im Einklang mit der vorliegenden Arbeit.

Gewissenhaftigkeit ist assoziiert mit Begriffen wie Organisation, Fleiß und Sorgfalt und vermittelt den Eindruck eines impulskontrollierten, gründlichen Menschen (Hoffman et al. 2010; Drosdeck et al. 2015). Menschen mit geringerer Gewissenhaftigkeit lassen sich als locker bis nachlässig klassifizieren, wobei dies ohne Wertung aufgenommen werden muss. Auch Extraversion und Verträglichkeit sind Eigenschaften, die in der effektiven Arbeit im Team unumgänglich sind. Sie sind assoziiert mit einer positiven, aktiven, durchsetzungsfähigen und sympathischen Ausstrahlung (Hoffman et al. 2010; Drosdeck et al. 2015).

Fraglich ist, ob von sogenannten chirurgischen Persönlichkeitsmerkmalen gesprochen werden kann, wenn von Entscheidungsfreude, Durchsetzungsfähigkeit, Autorität und Gewissenhaftigkeit (Thomas 1997; Hoffman et al. 2010) die Rede ist. Weiterhin ist diskutabel, ob sich Frauen in der Chirurgie diese Merkmale authentisch aneignen oder ob Frauen, die sich für die Chirurgie entscheiden, diese 
Persönlichkeitsstruktur besonders wünschen, weil das Streben nach dem NichtErreichten höher ist.

Skizziert man ein allgemeineres Bild der vorliegenden Ergebnisse, stimmen diese weitestgehend mit der Studie von Pololi et al. überein. Frauen und Männer teilten hier bezüglich Engagement und Enthusiasmus im Beruf übereinstimmende Ansichten. Frauen seien sich jedoch weniger selbstsicher bezüglich ihres Karrierefortschritts (Pololi et al. 2013). Diese Tatsache deckt sich mit dem Ergebnis der Arbeit von Schroen et al. und den Ergebnissen des "TransFerGenderMed“Projekts, die hervorhoben, dass Chirurgen, insbesondere in den Anfängen der Karriere, höhere berufliche Positionen anstrebten (Schroen et al. 2004; Uke.de Institut und Poliklinik für Allgemeinmedizin - Projekt Transfergendermed 2017).

Die akademische Medizin scheint ein Feld zu sein, in dem Stereotypen allgegenwärtig sind und dadurch möglicherweise ein Aufstieg von Frauen in leitende Positionen erschwert wird (Burgess et al. 2012). Diese behindernde Art von Stereotypie ist so definiert, dass Individuen als Teil einer Gruppe innerhalb eines bestimmten Bereiches einem negativen Stereotyp zugeordnet werden und sie allein aufgrund dieser Behauptung unterhalb ihrer Fähigkeiten lediglich im Bereich ihres zugeordneten Stereotypens agieren (Steele 1997; Burgess et al. 2012).

Sozialpsychologisch ist außerdem belegt, dass die eigene Wahrnehmung durch Geschlechterstereotype beeinflusst wird. Dies geschehe in der Weise, dass Männer sich in ihren Kompetenzen über-, Frauen sich tendenziell eher unterschätzen. Hinzukommt, dass Frauen stereotypisch eher als gefühlsbetont und fürsorglich, Männer als autonom, sachlich und lösungsorientiert gelten (Buddeberg-Fischer 2001; Burgess et al. 2012). Diese Aspekte betonten die weiblichen Teilnehmerinnen in den Freitextantworten, während die Männer u. a. die Empathie der Frauen hervorhoben und auf diese Weise die Stereotypen bestätigten. Das Bild des maskulinen, selbstbewussten nahezu einschüchternden Chirurgen ist noch präsent (Hill et al. 2014), befindet sich jedoch immer mehr im Wandel (Stabile 2008).

Erfolgreiche positive Rollenmodelle würden nicht nur eine Möglichkeit darstellen, das eigene Engagement zu stärken, sondern würden helfen, Vorurteile über die Chirurgie zu bekämpfen und die Stereotypie innerhalb der chirurgischen Disziplin zu verifizieren (Hill et al. 2014). 
Die Annahme, dass es Eigenschaften gäbe, die eine Karriere in der Chirurgie beeinflussen, kann bestätigt werden. Frauen maßen Charakteristika, die für Stärke stehen, mehr Gewichtung bei, sodass auch Geschlechtsunterschiede bestätigt werden konnten (Vergleiche Hypothese eins und zwei, Seite 8).

\subsubsection{Externe Karrierefaktoren}

Es wurde angenommen, dass es externe Karrierefaktoren gibt, die einen Einfluss auf eine chirurgische Karriere nehmen. Auch hier wurden Geschlechtsunterschiede erwartet.

Ein signifikanter Unterschied konnte bei den Items "hohe Fachexpertise“, „Ehrgeiz" und "Durchsetzungsvermögen" festgestellt werden, diese Items empfanden die Frauen durchschnittlich förderlicher. Das eigene "Geschlecht" wurde von den Frauen als hinderlich während des beruflichen Aufstiegs wahrgenommen, die Männer ordneten diesen Aspekt dem „Arbeitspensum“ und den „Arbeitszeiten“ unter.

Frauen bemerken allein aufgrund ihres Geschlechts eine Benachteiligung und fühlen sich schnell in eine Außenseiterposition gedrängt (Mayr 2008; Cochran et al. 2013; Seemann et al. 2016). Denkbar wäre, dass Frauen diese scheinbare Benachteiligung mit Persönlichkeitsstärken wie Ehrgeiz, Durchsetzungsvermögen und einer hohen Expertise ausgleichen, was ein Grund für die besondere Aufmerksamkeit auf diese Eigenschaften darstellen könnte.

Daneben dürfen rechtliche Grundlagen als externe Faktoren, gerade in Bezug auf die operative Arbeit während der Schwangerschaft nicht unberücksichtigt bleiben. Nicht nur im Ärzteblatt (Richter-Kuhlmann 2015) wird das Mutterschutzgesetz in Bezug auf die Beschäftigung im Gesundheitswesen in Frage gestellt, auch die Teilnehmenden der vorliegenden Studie forderten eine Umstrukturierung des Mutterschutzgesetztes, um auch bei Fortschreiten der Schwangerschaft operativ tätig bleiben zu dürfen. Rechtlich gesehen schließt das Mutterschutzgesetz eine operative Tätigkeit nicht aus, sodass Schwangeren dies unter optimalen Arbeitsschutzbestimmungen möglich gemacht werden könnte (Uni-luebeck.de - Leitfaden: Schwanger in der Chirurgie 2015). Leider ist noch häufig das Gegenteil der Fall, und es wird ein pauschales Einsatzverbot nach Bekanntgabe der Schwangerschaft ausgesprochen (Uniluebeck.de - Leitfaden: Schwanger in der Chirurgie 2015), was dazu führt, dass Chirurginnen in Ausbildung, die für die Weiterbildung geforderten Eingriffe nicht ableisten können. 
Insgesamt betrachtet kann das Infektionsrisiko durch moderne Instrumente, vor allem bei Durchführung von bestimmten Eingriffen wie Laparoskopien auf ein Minimum gesenkt werden (Wicker et al. 2011), weshalb sich zahlreiche Initiativen wie z. B. das Junge Forum der Gesellschaft für Unfallchirurgie zum Ziel gemacht haben, ein Beschäftigungsverbot für schwangere Chirurginnen zu umgehen und stattdessen eine rechtlich und gesundheitlich sichere Lösung für Arbeitgeber und Arbeitnehmer zu entwickeln (Uni-luebeck.de - Leitfaden: Schwanger in der Chirurgie 2015). In der Praxis müssen Vorschläge und ein Leitfaden dieser Art erst noch erprobt werden, die Universität Lübeck stellt mit dem sogenannten FamSurg-Programm ein erfolgreiches Praxisbeispiel dar (Chirurgie.uni-luebeck.de - FamSurg-Programm 2016). An dieser Stelle muss darauf hingewiesen werden, dass ab 01.01.2018 eine Änderung des Mutterschutzgesetztes in Kraft tritt und entsprechende Positionspapiere unter Umständen aktualisiert werden müssen (Bundesministerium für Familie, Senioren, Frauen und Jugend - Mehr Frauen profitieren künftig vom gesetzlichen Mutterschutz 2017).

Die Annahme, es gäbe Karrierefaktoren, die eine Karriere beeinflussen, kann bedingt bestätigt werden. Es gibt hinderliche und fördernde externe Faktoren während der beruflichen Laufbahn von Chirurginnen und Chirurgen, zwischen denen zusätzlich Geschlechtsunterschiede feststellbar waren. Entgegen den Erwartungen waren jedoch keine Geschlechtsunterschiede in Bezug auf externe Angebote und Maßnahmen (Vergleiche Tabelle 4) feststellbar. Es konnten erhebliche Defizite in der Bereitstellung von solch arbeitsplatzassoziierten Angeboten gezeigt werden, aber die Bewertungen dieser waren homogen zwischen den Geschlechtern (Vgl. Hypothese eins und zwei, Seite 8).

\subsubsection{Einfluss von Elternschaft, Familie und Schwangerschaft auf die}

\section{Karriereentwicklung}

In der vorliegenden Arbeit war die Annahme, dass eine Elternschaft die chirurgische Karriere von Frauen und Männern beeinflusst.

Das Memorandum von Rapp-Engels et al. zur Verbesserung der beruflichen Entwicklung von Ärztinnen, welches die Ergebnisse der Arbeitstagung "Geschlechterspezifische Perspektiven und Umsetzungsstrategien in der fachärztlichen Weiterbildung“ mit Bezugnahme auf die "KarMed“ Studie der 
Universität Hamburg (Uke.de - Institut und Poliklinik für Allgemeinmedizin Karriereverläufe von Ärztinnen und Ärzten in der fachärztlichen Weiterbildung (KarMed) 2017) zusammenfasst, macht deutlich, dass es noch immer eine Diskriminierung aufgrund des Geschlechts und des damit verbundenen "Risikos" der Schwangerschaft gebe (Rapp-Engels et al. 2012). Auch in der vorliegenden Arbeit hatten die Frauen durchschnittlich weniger Kinder, waren älter bei der Geburt des ersten Kindes und lebten häufiger allein als die männlichen Teilnehmer. Erstaunlicherweise schlossen jedoch die Mütter die Promotion früher ab als die Väter, was eventuell auf einen möglichen noch anstehenden Kinderwunsch zurückzuführen ist und den daraus resultierenden Zeitdruck einer zügigen Promotion. Hinzukommt die bis 2011 geltende Wehrpflicht/Zivilpflicht für die Männer, die einen späteren Studienbeginn verursachte. Entgegen einiger Literatur (Levinson et al. 1989; Willett et al. 2010; van den Bussche 2012; Chen et al. 2013; Uke.de - Institut und Poliklinik für Allgemeinmedizin - Projekt Transfergendermed 2017) konnte im Vergleich zwischen den kinderlosen Chirurginnen und Chirurgen dieser Umfrage und den Eltern keine zeitliche berufliche Verzögerung zwischen den verschiedenen Abschnitten der Karriere festgestellt werden. Die Teilnehmenden befanden sich möglicherweise in einer Position, in der es sowohl finanziell als auch personell möglich zu sein schien, eine Kinderbetreuung zu finanzieren. Die private Organisationsstruktur ist in dieser Phase der beruflichen Laufbahn häufig bereits so ausgearbeitet, dass die Eltern weniger Behinderungen durch die Familie im Beruf erfahren (Schroen et al. 2004). In einer Umfrage unter weiblichen und männlichen Chirurginnen und Chirurgen in Neuseeland und Australien wurde bestätigt, dass die Frauen, die sich bereits in einer akademischen Position befinden, durchaus eine kompatible Familien- und Arbeitsvereinbarkeit in der Chirurgie sehen (Saunders et al. 2008). Es scheint, als müsse die Phase während der fachärztlichen Ausbildung überbrückt werden, bis eine Familienplanung realistisch und nicht ausschließlich mit Stress verbunden ist (Mache et al. 2015). Einen anderen Standpunkt vertreten Carty et al., die in inrer Arbeit behaupten, dass Schwangerschaft und Kindererziehung während der Facharztausbildung mit ausreichender Unterstützung möglich und profitabel für alle Beteiligten sein könne (Carty et al. 2002).

Nichtsdestoweniger verlagern Frauen, die sich für eine Karriere in der Chirurgie entschieden haben, die Familienplanung zeitlich nach hinten, um die Karriere nicht 
zu gefährden (Schroen et al. 2004). Viele Medizinerinnen wollen die Schwangerschaft auf die Zeit nach der Facharztausbildung verlegen (Willett et al. 2010), was dazu führt, dass die Frauen Kinder gar nicht erst bekommen (Frank et al. 1998; Troppmann KM et al. 2009). Männer sind auf diese zeitliche Verlagerung oft nicht angewiesen, da sie Unterstützung durch den Partner oder die Partnerin erfahren, was sich mit den Angaben der vorliegenden Arbeit deckt. Auch in dieser Arbeit erfahren die Väter mehr Unterstützung in der Kinderbetreuung durch die Partnerin oder den Partner. Die Geburt des ersten Kindes fällt lediglich bei drei Teilnehmerinnen in die Zeit während der fachärztlichen Weiterbildung, bei den Männern sind es 26 Teilnehmer, die ein Kind während dieser Zeit bekommen. Problematisch ist, dass Frauen dadurch oft einen Konflikt Ihrer eigenen Karriere mit der Karriere des Partners erleben (Dyrbye et al. 2011). Die Partnerinnen und Partner der Frauen befinden sich oft in einer Vollzeitbeschäftigung, die der Männer eher halbtags (van den Bussche 2012), sodass die Frauen seltener die Möglichkeit haben, die Betreuung der Kinder an die Partnerin oder den Partner zu übergeben, was die vorliegenden Ergebnisse bestätigen.

Die Organisationstruktur in der Medizin, aber auch in anderen Fachbereichen und in der Wirtschaft ist häufig so aufgebaut, dass die Partnerinnen und Partner den Ehemännern eine so große Unterstützung gewähren, so dass sie fast keinerlei Abstriche im Beruf machen müssen (Schroen et al. 2004) und bei Elternschaft oft sogar mehr Arbeiten, was die Karriere wiederum fördert (Gibis et al. 2013; Römer et al. 2017). Dieses Geschlechterrollenmodell wirkt sich auf die Berufszufriedenheit aus (Bohrer et al. 2011), indem der Elternstatus die Zufriedenheit bei Ärztinnen negativ, bei Ärzten eher positiv beeinflusst (Römer et al. 2017). Demgegenüber stehen die Ergebnisse von Mache et al., nach denen keine Geschlechtsunterschiede in Bezug auf die Vereinbarkeit von Familie und Arbeit feststellbar sind und ein harmonisches und zufriedenes Arbeitsverhältnis, wozu z. B. ein guter Kontakt zu Vorgesetzten gehört, protektiv gegen eine Familien- und Arbeitsinkompatibilität wirke (Mache et al. 2015).

Richtet man den Blick gezielt auf einen Vergleich zwischen Müttern und Vätern kann das skizzierte Bild bestätigt werden. Auch in dieser Gruppe besetzen die Mütter insgesamt öfter einen Halbtagsjob und beschäftigten sich in der Arbeitszeit 
signifikant mehr mit Lehre und konservativen Tätigkeiten (z. B. Sprechstunden) als die Väter der Umfrage.

Neben dem Beruf kümmerten sich auch die Mütter zu einem Großteil selbst um die Kinder, was in aktueller Literatur bestätigt werden kann (Mayer et al. 2001; Uke.de Institut und Poliklinik für Allgemeinmedizin - Projekt Transfergendermed 2017). Viele Frauen unterliegen scheinbar allzu oft der Angst, traditionelle Rollenmuster zu überwinden und nehmen diese Doppelbelastung in Kauf (Yedidia und Bickel 2001; Dyrbye et al. 2011). Andere wiederrum entscheiden sich für einen Abbruch der akademischen Karriere, weil die hohe Arbeitsbelastung und die Arbeitszeiten häufig ein Problem darstellen (Mayer et al. 2001; Sonnad und Colletti 2002). Dieses spiegelt sich ebenfalls in den Antworten der Eltern unter den Teilnehmenden dieser Umfrage wieder, welche sich während ihres beruflichen Aufstiegs stärker durch die Arbeitsbelastung behindert fühlten, als die Teilnehmenden ohne Kinder. Eine regelmäßige Rund-um-die-Uhr-Betreuung, vor allem im Krankheitsfall der Kinder, würde das Problem zu weiten Teilen lösen können, dies wurde auch in den Freitextantworten betont. Es existieren bereits viele Praxisbeispiele, wie z. B. die Kindernotfallbetreuung des Berliner Alexianer Krankenhauses Hedwigshöhe, welches seinen Mitarbeiterinnen und Mitarbeitern die Möglichkeit einer Notfallkinderbetreuung an 365 Tagen im Jahr bietet (Marburger Bund - Für ein familienfreundliches Krankenhaus 2014). Beispiele wie dieses könnten als Muster für eine Umstrukturierung dienen, ein Langzeitnutzen muss allerdings noch abgewartet und evaluiert werden.

Durch vorliegende Ergebnisse konnte ein Einfluss der Elternschaft bestätigt werden. Es kann angenommen werden, dass Schwangerschaft und Kindererziehung eine Karriere vor allem während der fachärztlichen Ausbildung zeitlich verzögern und die zeitliche Stagnation vor allem bei den weiblichen Elternteilen dokumentiert werden kann. Bei bereits fortgeschrittener Karriere spüren ebenfalls die Mütter die beruflichen Nachteile stärker, insgesamt scheint jedoch der negative Einfluss wesentlich geringer als während der Assistenzarztausbildung (Vergleich Hypothese drei, Seite 8). 


\subsection{Work-Life-Integration und Zufriedenheit in akademischer Chirurgie}

Es wurde angenommen, es gäbe Faktoren, die eine berufliche Zufriedenheit beeinflussen und damit zu einer ausgeglichenen Work-Life-Balance führen.

Der Begriff Work-Life-Balance ist in der heutigen Zeit allgegenwärtig. Die Work-LifeBalance bezeichnet eine zufriedenstellende und ausreichende Kombination von Privatleben und Berufsalltag. Der Zusammenhang zwischen der beruflichen Zufriedenheit von Ärztinnen und Ärzten und dem Behandlungserfolg der Patienten (Wallace et al. 2009; Mache et al. 2012), setzt das Wissen der Faktoren voraus, die eine ärtliche Zufriedenheit ausmachen.

Einer Umfrage von Rosta und Gerber zufolge empfanden sich die Klinikärzte in Deutschland 2006 als „mäßig zufrieden“. Differenziert nach Fachrichtungen gaben die Chirurginnen und Chirurgen hier den niedrigsten Wert an. Letzteres wird durch die Ergebnisse von Römer et al. bekräftigt, Assistenzärztinnen und -ärzte in der Unfallchirurgie/Orthopädie gaben in dieser Studie die niedrigste Berufsverlaufszufriedenheit an (Römer et al. 2017). Als Faktoren, die eine mangelnde berufliche Zufriedenheit fördern, wurden fehlende Bestätigung in Form von finanzieller Entschädigung und positiven Rückmeldungen identifiziert (Rosta und Gerber 2008). Entgegen diesen Ergebnissen offenbart die vorliegende Studie, dass weibliche und männliche habilitierte Chirurginnen und Chirurgen zufrieden mit ihrem Beruf und ihrer Lebensqualität sind.

Zusammenfassend betrachtet, waren die besonders zufriedenen Teilnehmenden signifikant älter zum Zeitpunkt der Umfrage, jedoch jünger bei der Habilitation. Außerdem ließ sich eine relativ höhere Anzahl von Chefärztinnen und Chefärzte unter den besonders zufriedenen Teilnehmenden finden, was darauf schließen lässt, dass eine höhere berufliche Position häufig mit höherer beruflicher Zufriedenheit einhergeht (Buxel 2012). Die beruflichen Gratifikationskrisen einer hohen Anzahl chirurgischer Assistenzärztinnen und -ärzten bestätigt dies (Klein et al. 2010). Die besonders zufriedenen Teilnehmenden beschäftigten sich weniger mit konservativer Arbeit und mussten weniger Präsenzdienste pro Monat absolvieren, was ebenfalls häufig erst bei einer höheren beruflichen Position der Fall ist. Die Ergebnisse decken sich mit denen von Frank et al., die amerikanische Ärztinnen zur beruflichen Zufriedenheit befragten (Frank et al. 1999). 
Einer großer Teil der Chirurginnen und Chirurgen würden sich trotzdem wieder für eine Karriere in der Chirurgie entscheiden und einem Medizinstudierenden diesen Beruf weiterempfehlen (Schroen et al. 2004; Wyrzykowski et al. 2006). In einer großangelegten amerikanischen Studie wurde unter Chirurginnen und Chirurgen eine allgemeine Zufriedenheit gemittelt über alle chirurgischen Subdisziplinen von $68 \%$ festgestellt (Wai et al. 2014). Als die wichtigsten Prädiktoren wurden die Abteilungsführung, Kollegialität und Zusammenarbeit und das Verhältnis zu den Vorgesetzten benannt. Letzteres konnte in der vorliegenden Studie durch einen positiven Zusammenhang zwischen beruflicher Zufriedenheit der Frauen und Unterstützung durch den Vorgesetzten bestätigt werden. Viele Frauen in akademischen Positionen erfahren weniger Unterstützung durch die Institution und haben seltener die Möglichkeit, ein berufliches Netzwerk auszubauen (Kaplan et al. 1996), sodass sie als Konsequenz abhängig vom direkten Kontakt zu Vorgesetzen, Kolleginnen und Kollegen sind (Hitchcock et al. 1995).

Janus et al. rückten außerdem in den Vordergrund, dass nicht-finanzielle Determinanten einen entscheidenden Einfluss auf die Zufriedenheit von Ärztinnen und Ärzten haben, wozu z. B. ausreichend Lob und Anerkennung (BuddebergFischer et al. 2008) und das selbstständige Treffen von Entscheidungen gehöre (Janus et al. 2007). Dieses kann durch die vorliegende Studie und der Arbeit von Mache et al gestützt werden (Mache et al. 2012).

In der Studie von Ramirez et al. gaben die Chirurginnen und Chirurgen im Vergleich zu anderen Fachdisziplinen das größte Stresslevel an, obwohl sie die meisten Punkte erfültten, die für eine berufliche Zufriedenheit sprechen. Darin inbegriffen war z. B., dass Chirurginnen und Chirurgen im Vergleich zu anderen Fachdisziplinen durch direktes Feedback der Patienten, einen direkten Einfluss auf den Behandlungserfolg des Patienten haben (Ramirez et al. 1996). Eine erfolgreiche Behandlung ist in der Medizin oft assoziiert mit fachlicher Expertise der Ärztin oder des Arztes. Diese wiederum steht in nahezu direktem Verhältnis zu manuellem Geschick (Buckley et al. 2013; Bishawi und Pryor 2014; Buckley et al. 2014), welches in dieser Arbeit ebenfalls als ein positiver Prädiktor für berufliche Zufriedenheit identifiziert werden konnte. Grundsätzlich sollte zur Förderung der beruflichen Zufriedenheit Wert auf eine autonome Arbeit, ein gut strukturiertes 
Arbeitsumfeld und ein angenehmes Betriebsklima gelegt werden (Ramirez et al. 1996; Buxel 2012).

Eine hohe Berufszufriedenheit korreliert mit einer hohen Lebenszufriedenheit (Römer et al. 2017), was annehmen lässt, dass private Gegebenheiten einen ebenso entscheidenden Einfluss auf die berufliche Zufriedenheit haben, wie die beruflichen Strukturen. Die Berufstätigkeit des Partners oder der Partnerin beeinflusste negativ die Zufriedenheit der teilnehmenden Chirurgen, was ein Beispiel dieses Einflusses darstellt. Entgegen der geringen Berufsstundenzahl der Partnerinnen und Partner der teilnehmenden Männer in dieser Arbeit, werden jedoch generell betrachtet immer mehr dual-career-Partnerschaften eingegangen, in denen auch die Männer sich häufiger in der Vaterrolle sehen und bereit sind, die Arbeitszeit zugunsten der Kinder zu minimieren (Kwong et al. 2014). Dieser Trend muss durch flexiblere Arbeitszeitmodelle und einen Ausbau von Kinderbetreuungsangeboten unterstützt werden (Buddeberg-Fischer und Stamm 2010; Römer et al. 2017).

Die Teilnehmenden der vorliegenden Umfrage waren generell zufrieden mit ihrer privaten und beruflichen Situation. Die Annahme, dass Faktoren existieren, die die berufliche Zufriedenheit beeinflussen, kann bestätigt werden (Vergleiche Hypothese vier, Seite 8). Ein funktionierendes Privatleben mit einer ausgebauten Organisationsstruktur gehört ebenso dazu, wie ein Arbeitsumfeld welches ein autonomes Arbeiten und eine Unterstützung durch Vorgesetzte sicherstellt.

\subsection{Notwendigkeit karrierebeeinflussender Maßnahmen im Arbeitsumfeld}

Die Teilnehmenden wurden nach allgemeinen Veränderungen gefragt, die sie sich von Ihrem Arbeitgeber wünschen. Die Antworten konnten in vier Subkategorien eingeteilt werden (vgl. Tabelle 5). In Bezug auf unterstützende Angebote und Bedingungen, wie z. B. verlässliche Arbeits- und Urlaubszeiten, Mentoring, ausreichend Kinderbetreuungsmöglichkeiten, Karriereplanungen und flexible Arbeitszeitmodelle konnten in dieser Arbeit keine signifikanten Unterschiede zwischen Frauen und Männern festgestellt werden. In anderen Arbeiten hatten die Männer hingegen generell einen besseren Eindruck von der institutionellen Unterstützung (Sonnad und Colletti 2002), speziell in Hinblick auf eine Förderung 
durch Vorgesetzte (Uke.de - Institut und Poliklinik für Allgemeinmedizin - Projekt Transfergendermed 2017).

Kass et al. stellten während Interviews mit beruflich fortgeschrittenen Chirurginnen fest, dass viele Führungsfähigkeiten erlernt und vertieft werden können und weniger von Eigenschaften der Persönlichkeit abhängen (Kass et al. 2006). Umso erstaunlicher ist es, dass die Teilnehmenden der vorliegenden Studie in den meisten Fällen nicht die Möglichkeit erhielten, ihre Führungskompetenzen institutionell weiterzuentwickeln.

Neben diesen extrinsischen Aspekten, müssen strukturelle Veränderungen wie verlässliche Arbeits-und Urlaubszeiten, ein erleichterter Wiedereinstieg nach der Elternzeit, flexible Arbeitszeitmodelle und Betreuungsmöglichkeiten für Kinder ausgebaut werden, damit eine Verzahnung zum Privatleben möglich ist. Als Konsequenz muss eine Verbesserung der Arbeitsbedingungen im personellen, interpersonellen und strukturellen System gefordert werden (Saunders et al. 2008; Klein et al. 2010).

Diese Flexibilität sollte bereits während der Facharztausbildung beginnen. Eine strukturierte Weiterbildungsordnung mit integrierten wissenschaftlichen universitären oder außeruniversitären Forschungshospitationen (Stroh 2010) und zielorientierter Laufbahnberatung (Buddeberg-Fischer et al. 2008) würde beruflicher Unzufriedenheit vorbeugen (Buxel 2009). Es müsse insbesondere eine Perspektivenklarheit vorhanden sein, betonen auch die Teilnehmenden der vorliegenden Studie. Die Perspektivenklarheit wäre deutlicher wahrzunehmen, wenn sich die Fortbildung nicht an Zeit oder einem Operationskatalog orientiert, sondern wesentliche Lernziele und Lernfortschrittskontrollen in den Fokus gelangen (Römer et al. 2017).

Mit zunehmendem Frauenanteil in der medizinischen Profession ändert sich nicht nur die Arbeits- und Verhaltensweise, sondern auch das zur Verfügung stehende Arbeitsvolumen (Bundesärztekammer und Kassenärztliche Bundesvereinigung Dem deutschen Gesundheitswesen gehen die Ärzte aus! Studie zur Altersstruktur und Arztzahlentwicklung 2010). Es existiert insgesamt ein Trend zur Arbeitszeitverkürzung, Ärztinnen absolvieren hierbei insgesamt weniger Arbeitsstunden als die Ärzte (Bundesärztekammer und Kassenärztliche Bundesvereinigung - Dem deutschen Gesundheitswesen gehen die Ärzte aus! Studie zur Altersstruktur und Arztzahlentwicklung 2010). Einer der Gründe dafür liegt 
sicherlich am zunehmenden Interesse einer Teilzeitbeschäftigung (Römer et al. 2017). Obwohl auch die Teilnehmerinnen dieser Umfrage durchschnittlich weniger Wochenarbeitsstunden absolvierten als die Männer, empfanden sie "Teilzeitarbeitsmodelle" als hinderlicher für den beruflichen Aufstieg. Dies spiegelte sich auch in Freitextantworten wider, in denen eine Teilnehmerin erläuterte, dass sie sich bereits bei "Studienbeginn für die Karriere und gegen die Familie entschieden“ habe. Zwei andere Teilnehmerinnen betonten, dass eine "Chefarzttätigkeit und damit verbundene akademische Chirurgie in Teilzeit nicht sinnvoll sei“. Dies liegt mutmaßlich zum einen an faktischen Nachteilen wie einem geringen Einkommen und einer längeren Weiterbildungszeit, zum anderen an der Vorstellung, dass gute Ärztinnen und Ärzte ihr Engagement durch eine lange Anwesenheit in der Klinik zeigen (Rapp-Engels et al. 2012). Dass eine sogenannte Präsenzkultur in der Klinik mehr Wertschätzung erfahre als "qualitative Arbeit" (Zitat Freitextantworten) wurde auch von einer Teilnehmenden beanstandet. Im Memorandum zur Verbesserung der beruflichen Entwicklung von Ärztinnen von Rapp-Engels et al., welches die Ergebnisse der Arbeitstagung "Geschlechterspezifische Perspektiven und Umsetzungsstrategien in der fachärztlichen Weiterbildung" zusammenfasst, wird somit eine effektive akademische Teilzeitbeschäftigung angezweifelt. Mit zunehmendem Dienstrang sei eine Teilzeitbeschäftigung immer weniger realisierbar, so die gängigen Vorstellungen (Rapp-Engels et al. 2012). Obwohl bei vielen Ärztinnen und Ärzten trotzdem zunehmendes Interesse an Teilzeitbeschäftigungen besteht (Ziegler et al. 2017), werden bereits vorhandene Maßnahmen zur flexiblen Arbeitszeitgestaltung sowohl von Arbeitnehmer- als auch von Arbeitgeberseite nicht im ausreichenden Maße angenommen (Rapp-Engels et al. 2012). Im Gegensatz dazu empfand ein großer Teil der Teilnehmerinnen der Studie zur Berufs- und Lebenssituation Salzburger Ärztinnen Teilzeitarbeitsmodelle als sinnvoll, sofern ein Verständnis der Kollegen bestünde (Mayr 2008). C. Stroh identifiziert dies ebenfalls als eine Möglichkeit, dem chirurgischen Fachärztemangel entgegenzuwirken (Stroh 2010). Vermutlich könnten mit der Möglichkeit einer Teilzeitbeschäftigung mehr Frauen für die Chirurgie begeistert werden, unter Berücksichtigung der Analyse der Bundesärztekammer müsste das anfallende Arbeitsvolumen aber wiederum auf eine größere Anzahl von Ärztinnen und Ärzte verteilt werden und würde so den Fachärztemangel verschärfen (Bundesärztekammer und Kassenärztliche 
Bundesvereinigung - Dem deutschen Gesundheitswesen gehen die Ärzte aus! Studie zur Altersstruktur und Arztzahlentwicklung 2010).

$\mathrm{Zu}$ weiteren unterstützenden Maßnahmen gehören Mentoren und Dozenten, die sowohl Hilfestellung leisten könnten, als auch eine Vorbildfunktion darstellen. Solch eine Vorbildfunktion erleichtert nicht nur den Einstieg, sondern fördert auch die Entwicklung einer Karriere (Yedidia und Bickel 2001; Riska 2011; Seemann et al. 2016). Frauen haben jedoch selten die Möglichkeit, weibliche Kolleginnen in höheren Positionen zu beobachten (Nonnemaker 2000). Dieser Zustand verringert wiederum das Selbstvertrauen der Frauen und vermittelt ein Gefühl von Resignation. Ein gutes Beispiel stellt das Projekt „Frauenwege-Mut sichtbar machen“ der Universität Wien dar, in dem in regelmäßigen Abständen beispielhaft Karrierewege von Wissenschaftlerinnen der Uni Wien dargestellt werden. Hier wird auf Motivationslagen, Karriereverläufe, persönliche Stärken/Schwächen und Barrieren im Karriereverlauf Bezug genommen (Medizinische Fakultät Universität Wien Frauenwege - Mut sichtbar machen 2006).

Erstaunlicherweise wurde von den Teilnehmenden der vorliegenden Studie „Unterstützung durch den Vorgesetzten“ und „Networking“ im Vergleich zu den anderen Items als weniger wichtig eingeschätzt. Die gängige Meinung lautet jedoch, dass Mentoring ein wichtiger Faktor für die Karriereentwicklung von Männern und Frauen darstellt (Colletti et al. 2000) und das persönliche Gespräch und der Austausch von Erfahrungen unverzichtbar ist (Hoover 2006). Gespräche dieser Art, die im besten Falle auf gleicher Augenhöhe stattfinden, könnte möglicherweise die hierarchische Struktur mindern (Sexton et al. 2012) und eine Verzögerung der Karriere durch eine bestehende Rangordnung vermeiden (Conrad et al. 2010). Darüber hinaus gibt es bereits eine Reihe von Programmen, die sich zur Aufgabe genommen haben, Berufsanfängerinnen und Berufsanfängern Führungsqualitäten zu vermitteln. Hierzu zählt z. B. das „Duke Medicine Management and Leadership Pathway", welches seit 2010 Assistenzärztinnen und Assistenzärzten sowohl medizinisches Hintergrundwissen als auch Führungsqualitäten vermittelt, sodass das Erreichen einer Führungsposition nicht ausschließlich durch Zufall geschieht (Ackerly et al. 2011). 
Die Annahme, es gäbe Faktoren, die eine Karriere in der Chirurgie beeinflussen, kann bestätigt werden (vgl. Hypothese eins, Seite 8). Entgegen gängiger Literatur, konnten in der Inanspruchnahme von karrierebeeinflussenden Angeboten durch den Arbeitgeber jedoch keine geschlechtsspezifischen Unterschiede festgestellt werden (vgl. Hypothese zwei, Seite 8). Die Möglichkeit der Teilzeitarbeit findet in der Allgemeinheit zunehmendes Interesse, ist aber bei fortgeschrittener Karriere schwer realisierbar, ebenso scheint z. B. Mentoring bei Fortschritt der Karriere weniger Resonanz zu finden, als während der Weiterbildungszeit.

\subsection{Stärken und Schwächen der Studie}

Dies ist eine der ersten Querschnittumfragen unter habilitierten Chirurginnen und Chirurgen in Deutschland. Betrachtet man die Stärken der Studie, handelte es sich zum einen um eine nationale Umfrage im multi-institutionellen Bereich, zum anderen um eine große repräsentative Stichprobe mit einer für den medizinischen Bereich vergleichsweisen hohen Rücklaufquote. Die vorliegende Arbeit liefert einen detaillierten Überblick der biografischen Daten und zahlreiche Einschätzungen zu Einstellungen, Werten und Arbeitsbedingungen der Teilnehmenden. Hinderliche und förderliche Faktoren während der Karriereentwicklung und des beruflichen Fortschritts wurden zusammengefasst.

Nichtsdestoweniger ist diese Umfrage nicht repräsentativ, sondern basiert lediglich auf einer Auswahl an habilitierten Chirurginnen und Chirurgen in Deutschland. Ferner können selbstredend in einer Studie nicht alle Faktoren berücksichtigt und identifiziert werden, die eine Karriere in der Chirurgie fördern, stärken und behindern. Es besteht lediglich die Annahme, dass alle deutschen habilitierten Chirurginnen erreicht wurden, da die Datenschutzrichtlinien der Fachgesellschaften eine Herausgabe der Kontakte nicht zulässt und keine offizielle Liste aller habilitierten Chirurginnen existiert. Eine andere Unsicherheit besteht darin, dass die männlichen Teilnehmer auf Grundlage der weiblichen Teilnehmerinnen ausgewählt werden mussten, um einer matched-pair-Analyse gerecht zu werden. Die Tatsache, dass sich die Teilnehmenden in den meisten entscheidenden soziodemografischen Aspekten nicht unterscheiden, spricht allerdings für die Selektion der Teilnehmer, wenngleich es eine Selektion bleibt. 
Die mutmaßliche höhere Rücklaufquote der Frauen bestätigte sich. Bei den Frauen lag diese bei $67,7 \%$, während die der männlichen Subgruppe bei etwa der Hälfte lag (35\%). Der Mittelwert beider Quoten (51\%) lässt sich mit anderen medizinischen Erhebungen durchaus vergleichen (Linn LS et al. 1985; Lowenstein et al. 2007; Cropsey et al. 2008).

Obwohl vor der Erstellung des Fragebogens eine umfangreiche Literaturrecherche durchgeführt wurde, ein kritisches interdisziplinäres Debriefing zu den formulierten Items des Fragebogens stattfand sowie Testdurchläufe durchgeführt wurden, meldeten einige Teilnehmende Probleme zum Verständnis der Fragen zurück.

Es wurde angemerkt, dass „relevante Fragen missverständlich ausgedrückt“ seien, weil Items nicht eindeutig formuliert waren. Obwohl die Möglichkeit bestand, die Antwortoption „entfällt" zu wählen, war dieser Punkt für einige Teilnehmenden wohl nicht eindeutig, sodass Teilnehmende sich für einen Faktor entschieden, obwohl sie selbst keinen Nutzen durch diesen hatten.

Hinzukommt, dass als Email-Umfrage die Gefahr der sogenannten Schweigeverzerrung (engl. non-response bias) besteht, wobei die Tragweite dieser Verzerrung bei Umfragen unter Medizinerinnen und Medizinern nicht so ausschlaggebend ist, wie bei Umfragen unter der Allgemeinbevölkerung (Kellerman 2001). Des Weiteren gibt es Hinweise darauf, dass eine daraus resultierende NichtRepräsentativität der Daten ein nicht so großes Problem darstellt, wie bisher angenommen (Siemiatycki und Campbell 1984; Asch et al. 1997; Axinn et al. 2011). Obwohl die Möglichkeit einer Schweigeverzerrung besteht, kann die Repräsentativität einer Umfrage u. a. durch eine ausreichende Rekrutierung und eine gezielte Übervertretung (engl. Oversampling) einer Teilgesamtheit gesteigert werden (Corry et al. 2017), was in der vorliegenden Studie der Fall ist. 


\section{$5 \quad$ Zusammenfassung}

Fragestellung und Zielsetzung: Aufgrund eines steigenden Anteils weiblicher Medizinstudierender wird in der Literatur häufig von einer „Feminisierung“ des Arztberufes gesprochen. Bei Betrachtung des Fachgebietes der akademischen Chirurgie kann von solch einer Feminisierung keine Rede sein, es zeichnet sich hingegen ein Nachwuchsmangel ab. Ausgehend von dem Missverhältnis zwischen Personalbedarf und Personalmangel in der akademischen Chirurgie sollte in dieser Studie ein Überblick über die aktuelle Arbeits- und Lebenssituation und die subjektive Einschätzung zu Karrierefaktoren der habilitierten Chirurginnen und Chirurgen in Deutschland erlangt werden. Mit Hilfe der vorliegenden Ergebnisse können Vorschläge zu Verbesserungen gemacht werden sowie Vor- und Nachteile der gegenwärtigen Verhältnisse, insbesondere in Bezug auf Karriereförderung, aufgezeigt werden.

Methode: Alle in Deutschland recherchierbaren, habilitierten Chirurginnen wurden zu dieser Querschnittsstudie eingeladen. Die Chirurgen wurden in Kongruenz der relevanten soziodemographischen Faktoren wie Arbeitsort, Größe des Arbeitsplatzes, Fachgebiet und beruflicher Qualifikation zu ihren weiblichen Kolleginnen identifiziert und im 2:1- Verhältnis eingeladen. Über einen Onlinelink erhielten die Teilnehmenden Zugänge zu den 103-Item Fragebögen. Zwischen Juli und September 2014 erfolgte die anonyme Datenerhebung mittels EvaSys $\AA$ (Lüneburg, Deutschland).

Ergebnisse: Der Fragebogen wurde an 93 Chirurginnen und 200 Chirurgen gesendet, von denen 63 Frauen (67,7\%) und 70 Männer (35,0\%) antworteten. Das Durchschnittsalter betrug 47,5 und 47,1 Jahre. Im Schnitt hatten die Frauen 1,47, die Männer 2,24 Kinder. Die Teilnehmenden empfanden die Items „hohe fachliche Expertise“, „Ehrgeiz“ und „Klarheit der eigenen Ziele“ als wichtige Faktoren für eine Karriere. Beide Gruppen empfanden das „Arbeitspensum“, die „Arbeitszeiten“ und das "Geschlecht" als hinderlich für den beruflichen Aufstieg, wobei Frauen letzteres als noch hinderlicher empfanden, $(p<, 05)$. "Belastbarkeit", „Verantwortungsbewusstsein“ und „eine gute Selbstorganisation“ wurden als wichtige Persönlichkeitsmerkmale erfasst, wobei die Frauen auch diesen Items mehr 
Gewichtung beimaßen $(p<, 05)$. Die Berufszufriedenheit wurde sowohl von den Chirurginnen $(69,5 \%)$ als auch von den Chirurgen $(75,5 \%)$ als hoch angegeben. „Unterstützung durch den Vorgesetzten“ ( $B=-, 41)$ und „manuelles Geschick“ ( $B=-, 41)$ wurden als Prädiktoren für eine besonders hohe berufliche Zufriedenheit $(90-100 \%)$ bei den Frauen identifiziert. Die Kinderbetreuung durch „Kindergarten/Hort/Schule“ wies den höchsten negativen $B$-Koeffizienten $(B=-1,33)$ auf.

Schlussfolgerung: Viele Vorstellungen und Meinungen von Chirurginnen und Chirurgen in Bezug auf nützliche und hinderliche Karrierefaktoren weisen Parallelen auf. Hierzu gehört u. a. die Notwendigkeit einer Betreuung und Förderung durch Vorgesetzte sowie eine fachliche Expertise, die durch ausreichend Forschungszeit und eine organisierte Weiterbildung gefördert werden könnte. Ebenso empfinden die Teilnehmenden bestimmte Persönlichkeitsmerkmale als förderlich für den beruflichen Weg, in diesem Zusammenhang muss vor allem Bezug auf Charakteristika genommen werden, die mit Stärke und Selbstsicherheit assoziiert sind. Nichtsdestoweniger nehmen Chirurginnen ihr Geschlecht noch immer als hinderlich für den beruflichen Aufstieg wahr, ebenso beeinflusst Kindererziehung und der Elternstatus eine Karriere und die berufliche Zufriedenheit. Um einem langfristigen Mangel an Fachärztinnen und Fachärzten entgehen, müssten Strategien entwickelt werden, um eine Gleichheit in der Karriereentwicklung zu ermöglichen. Langfristig sollte eine geschlechtsspezifische Berufsberatung entwickelt werden, zu der z. B. ein organisiertes Mentoring gehören sollte. Zusätzlich wäre eine Verbesserung der Organisations- und Arbeitsgestaltung, z. B. mit flexibleren und zuverlässigen Arbeitszeiten, wünschenswert. 
$6 \quad$ Anhang

6.1 Fragebogen, weibliche Fassung 


\section{MUSTER}

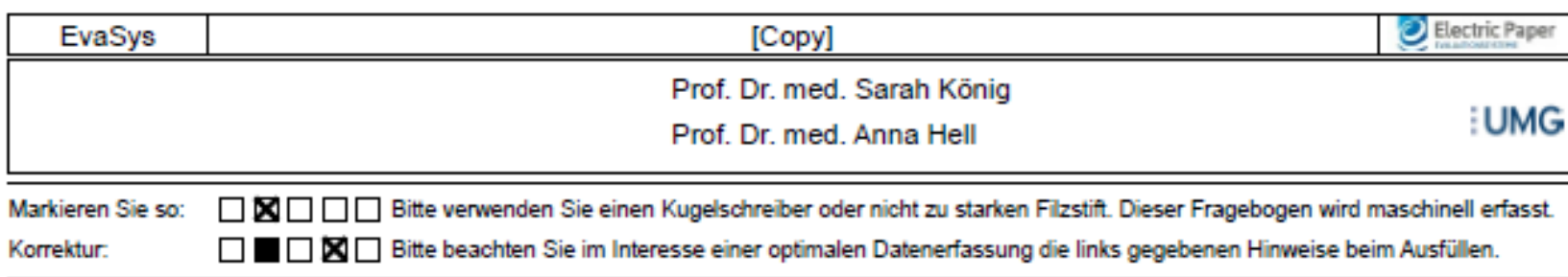

\section{Der erste Fragenblock bezieht sich auf allgemeine Angaben zu Ihrer Person und zu Ihrer derzeitigen Berufstätigkeit.}

1. Wie alt sind Sie? (Jahre)

2. In welchem Bundesland befindet sich Ihr Arbeitsort?
$\square$ Baden-Württemberg
$\square$ Bayern
$\square$ Brandenburg
$\square$ Bremen
$\square$ Hessen
$\square$ Mecklenburg-Vorpommern
$\square$ Berlin
$\square$ Nordrhein-Westfalen
$\square$ Rheinland-Pfalz
$\square$ Hamburg
$\square$ Sachsen
$\square$ Sachsen-Anhalt
$\square$ Niedersachsen
$\square$ Thüringen
$\square$ Saarland
$\square$ Schleswig-Holstein

3. Welche berufliche Position nehmen Sie gegenwärtig ein? (Mehrfachnennung möglich)
$\square$ W3/C4-Professur
$\square$ Chefärztin
$\square$ W2/C3-Professur
$\square$ Chirurgin mit
Schwerpunktleitung / Sektion
$\square$ W1-Professur
$\square$ Leitende Funktion als
$\square$ Fachärztin Oberärztin
Oberärztin
Sonstiges (bitte nennen):
$\square$ Assistenzärztin
4. Meine aktuellen Qualifikationen sind : a. Fachärztin für (Mehrfachnennung möglich)
$\square$ Allgemeinchirurgie
$\square$ Kinderchirurgie
$\square$ Gefäßchirurgie
$\square$ Orthopädie/Unfallchirurgie
$\square$ Herzchirurgie
Thoraxchirurgie
$\square$ andere (bitte nennen):
$\square$ Viszeralchirurgie
$\square$ Plastische und Ästhetische Chirurgie
$\square$ keine Facharztqualifikation

b. zusätzliche Weiterbildungen oder Zusatzbezeichnungen (bitte nennen):

c. weitere Abschlüsse in Aufbau-, Bachelor- oder Masterstudiengängen (bitte nennen): 


\section{MUSTER}

\section{Der erste Fragenblock bezieht sich auf allgemeine Angaben zu Ihrer Person und zu Ihrer derzeitigen Berufstätigkeit. [Fortsetzung]}

5. Mein aktuelles Tätigkeitsfeld erstreckt sich auf: (Mehrfachnennung möglich)

$\square$ ambulante Krankenversorgung/ Praxis (angestellt)

$\square$ Forschungseinrichtung

$\square$ Universitätsmedizin $\square$ ambulante Krankenversorgung/ Praxis (selbstständig)

$\square$ keine Erwerbstätigkeit

$\square$ Sonstiges (bitte nennen): $\square$ Elternzeit

Krankenhaus (außer Universitätsmedizin)

6. Bitte geben Sie Ihr Alter an, in dem Sie ihre Approbation erhalten haben:

7. Bitte geben Sie Ihr Alter bei Ihrer Promotion an:

8. Bitte geben sie Ihr Alter bei Ihrer Habilitation an:

9. Meine durchschnittliche Arbeitswoche (100\%) setzt sich prozentual aus folgenden Bereichen zusammen:

(Mehrfachnennung möglich, bitte vergeben Sie möglichst in 10\%-Stufen)

a. Forschung (in \%):

b. konservative Tätigkeiten (z.B. Sprechstunden) (in \%):

c. Lehre (in \%):

d. operative Tätigkeiten (eigenverantwortlich) (in \%):

e. Verwaltung, Gremien (in \%):

f. Sonstiges (bitte nennen, in \%):

10. Meine vertragliche wöchentliche Arbeitszeit (Regelarbeitszeit ohne Dienste) beträgt in Stunden:

11. Meine tatsächliche wöchentliche Arbeitszeit beträgt in Stunden im Durchschnitt: 


\section{MUSTER}

EvaSys

[Copy]

Der erste Fragenblock bezieht sich auf allgemeine Angaben zu Ihrer Person und zu Ihrer derzeitigen Berufstätigkeit. [Fortsetzung]

12. Meine durchschnittlich Anzahl von Präsenzdiensten im Monat beträgt:

13. Meine durchschnittliche Anzahl von Ruf- und Hintergrunddiensten im Monat beträgt:
14. Haben Sie einen Forschungsaufenthalt
$\square$ ja
$\square$ nein im Ausland von mindestens 6 Monaten absolviert?

\section{Der folgende Fragenblock beschäftigt sich mit Ihrem beruflichen und familiären Werdegang. \\ - Teil 1 -}

15. Bitte geben Sie die Anzahl Ihrer Kinder an $\square 0$

$\square 3$

\section{$\square 1$}

$\square 4$ $\square 2$

$\square>4$

-.--Fragen 16-19 sind nur von Ärztinnen mit Kindern zu beantworten--.

16. Bitte geben Sie Ihr Alter bei der Geburt Ihres ersten Kindes an:

17. In welcher Ausbildungs- bzw. beruflichen Position befanden Sie sich zum Zeitpunkt der Geburt Ihres ersten Kindes?

(Mehrfachnennungen möglich)

$\square$ vor $/$ während des Studiums

$\square$ nach dem Studium, jedoch vor der Facharztweiterbildung

$\square$ Fachärztin

$\square$ promovierte Ärztin $\square$ Oberärztin

$\square$ habilitierte Ärztin $\square$ während der fachärztlichen Weiterbildung

$\square$ leitende Position

18. Wie lange haben Sie nach der Geburt Ihrer Kinder insgesamt (Summe aller Kinder) komplett (keine vertragliche Arbeitszeit) beruflich pausiert?

Hinweis: Wenn Sie gar nicht pausiert haben (ausschließlich Mutterschutzzeit), tragen Sie bitte die Zahl 0 ein.

19. Wie viel Prozent der anfallenden Aufgaben im Bereich Kinderbetreuung (100\%) werden an einem typischen Werktag von den angeführten Personen/Institutionen übernommen?

a. von Ihnen (in \%): 


\section{MUSTER}

EvaSys

[Copy]

Der folgende Fragenblock beschäftigt sich mit Ihrem beruflichen und familiären

Werdegang.

- Teil 1 -

[Fortsetzung]

b. von Ihrer Partnerin/lhrem Partner (in \%):

d. von einer Betreuung im Haushalt (Kindermädchen, Au pair,...) (in \%):

c. von einem anderen Familienmitglied (in \%):

e. von einer Tagesmutter (in \%):

f. Kindertagestätte, Schule, Hort (in \%):

g. Sonstiges (bitte nennen, in \%):

Der folgende Fragenblock beschäftigt sich mit Ihrem beruflichen und familiären Werdegang.

- Teil 2 -

20. Wie stark haben Sie folgende Faktoren in Ihrer Berufswahl beeinflusst?

gute Aufstiegsmöglichkeiten (Karriere)

guter Verdienst

Ausübung einer Führungsposition

Ansehen/Prestige

Einfluss durch Dritte/Vorbilder/Verwandte

Kontakt mit Patienten/innen, Menschen helfen können

persönliche Entfaltungsmöglichkeiten

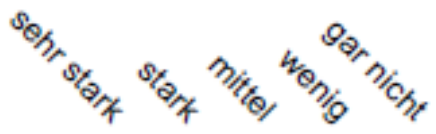

$\square \square \square \square \square$

$\square \quad \square \quad \square \quad \square \quad \square$

$\square \square \square \square \quad \square$

$\square \square \square \square \square$

$\square \square \square \square \quad \square$

$\square \quad \square \quad \square \quad \square \quad \square$ 


\section{MUSTER}

EvaSys

[Copy]

Der folgende Fragenblock beschäftigt sich mit Ihrem beruflichen und familiären

Werdegang.

- Teil 2 -

[Fortsetzung]

persönliche Fähigkeiten/ Eignung/Interessen

selbstbestimmtes und eigenverantwortliches

Arbeiten

gute Vereinbarkeit von Privatleben und Beruf

gute Weiterbildungsmöglichkeiten

Zufall

Sonstige Gründe (bitte nennen):

21. Wie hilfreich waren folgende Angebote/Bedingungen während Ihrer beruflichen Karriere?

Hinweis: Sie können die von Ihnen genutzten Angebote bewerten (hilfreich bzw. nicht hilfreich) oder angeben, warum diese nicht von Ihnen genutzt wurden (kein Bedarf am Angebot bzw. kein Angebot vorhanden)

- Angebote zur Vereinbarkeit von Familie und Beruf-

ausreichende

Betreuungsangebote für die

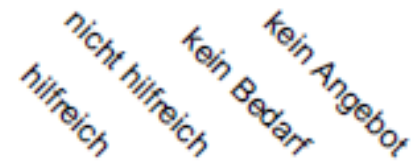

eigenen Kinder (z.B. betriebliche

Kindertagesstätte, 24 Stunden

Betreuung, Notfallbetreuung)

verlässliche Arbeitszeiten und

Urlaubsplanungen

erleichterter Wiedereinstieg nach

der Elternzeit (z.B.durch

Kontaktpflege während der Elternzeit

und Förderung des Wiedereinstiegs)

Teilzeitarbeitsmodelle

- Angebote zur Persönlichkeitsentwicklung/Persönlichkeitsbildung/ Karriereförderung- 


\section{MUSTER}

EvaSys

[Copy]

Der folgende Fragenblock beschäftigt sich mit Ihrem beruflichen und familiären

Werdegang.

- Teil 2 -

[Fortsetzung]

Karriereplanungen

(z.B. durch Fortbildungen,

Stipendien, Netzwerke und

Seminare, die teilweise speziell für

Frauen ausgelegt sind)

\section{Mentoring/Coaching}

Personalentwicklung/

Schlüsselkompetenzen

(z.B. Fortbildungen für weibliche

Führungskräfte; Angebote zu

Sprach-, Methoden-, Selbst-, und

Sozialkompetenzen)

\section{Supervision}

Nachwuchsförderung

Sonstiges (bitte nennen):

22. Welche Angebote zur Unterstützung (u.a. familienfreundliche Angebote) würden Sie sich von Ihrem Arbeitgeber noch wünschen?

23. Wie förderlich bzw. hinderlich fanden Sie folgende Faktoren für Ihren beruflichen Aufstieg?

Arbeitspensum

Arbeitszeiten (z.B. Nachtdienste)

Durchsetzungsvermögen

eine dem Ausbildungsstand

angemessene operative Tätigkeit

Ehrgeiz

F4374UOP6PLOVO

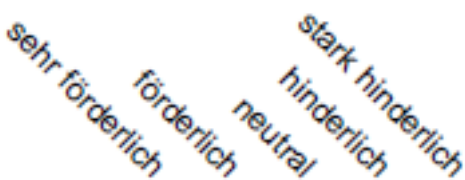

$\square \square \square \square \square$

$\square \quad \square \quad \square \quad \square \quad \square$

$\square \square \square \square \square$

$\square \quad \square \quad \square \quad \square \quad \square$

$\square \quad \square \quad \square \quad \square \quad \square$

\section{MUSTER}




\section{MUSTER}

EvaSys

[Copy]

Der folgende Fragenblock beschäftigt sich mit Ihrem beruflichen und familiären

Werdegang.

Teil 2 -

[Fortsetzung]

Elternzeit

hohe Fachexpertise

Flexibilität (in Bezug auf z.B.

Arbeitsort)

Geschlecht

Kenntnis der

Organisationsstrukturen

Kindererziehung

Klarheit der eigenen beruflichen

Ziele

Kollegialität

Networking, Vernetzung, Kontakte

Schwangerschaft

Teilzeit-Stelle (sog. „Präsenz-

Kultur $=$ lange Arbeitszeit= gute

Chirurgin)

Unterstützung durch Vorgesetzte

Gut strukturierte Weiterbildung

(strukturierter OP Plan, Logbuch,

Weiterbildungsgespräche mit

Vorgesetzten, etc.)

Sonstiges (bitte nennen):

Der folgende Fragenblock beschäftigt sich mit Ihrer Freizeitgestaltung und Ihrem Privatleben. 


\section{MUSTER}

EvaSys

[Copy]

Der folgende Fragenblock beschäftigt sich mit Ihrer Freizeitgestaltung und Ihrem Privatleben. [Fortsetzung]

24. Ist/War einer Ihrer Eltern Akademiker/in?

$\square$ ja, beide

$\square$ ja, ein

Elternteil

nein

25. Mein aktueller Familienstand ist:

$\square$ alleinstehend

$\square$ verheiratet

oder in

geschieden, lebend oder verwitwet

-.--Frage 26-27 sind nur von Ärzten/Ärztinnen mit Partner/in zu beantworten--_-

26. Welchen Beruf übt Ihr/e Partner/in aus?

27. Wie viele Stunden ist Ihr/e Partner/in pro Woche aktuell berufstätig? Hinweis: Bei keiner Erwerbstätigkeit tragen Sie bitte die Zahl 0 ein.

28. Mein gegenwärtiges Zeitbudget $(\mathbf{1 0 0 \%})$ in einer durchschnittlichen Woche (inkl. Wochenende) verteilt sich prozentual auf folgende Lebensbereiche:

a. Beruf (in \%)

b. ehrenamtliche Tätigkeit (in \%):

c. Familie (in \%):

d. Freizeitaktivitäten, Hobbies, Freunde (in \%):

e. Haushalt (in \%):

f. Sonstiges (bitte nennen, in \%):

Der folgende Fragenblock erfasst Ihre berufliche und allgemeine Zufriedenheit, sowie Ihre Selbsteinschätzung.

\section{MUSTER}




\section{MUSTER}

\begin{tabular}{l|l} 
EvaSys & [Copy]
\end{tabular}

Der folgende Fragenblock erfasst Ihre berufliche und allgemeine Zufriedenheit, sowie Ihre Selbsteinschätzung. [Fortsetzung]

29. Wie wichtig sind/waren Ihrer Meinung nach, folgende Eigenschaften für Ihre eigene berufliche Karriere in der Chirurgie?

Anpassungsfähigkeit

Belastbarkeit

Durchsetzungsvermögen

emotionale Stabilität

Empathie

Entscheidungsfreude

Extraversion

Fleiß

Gewissenhaftigkeit

manuelles Geschick

Offenheit für Neues

Problemlösefähigkeit

Risikobereitschaft

Selbstbewusstsein

gute Selbstorganisation

Verantwortungsbewusstsein

Verträglichkeit

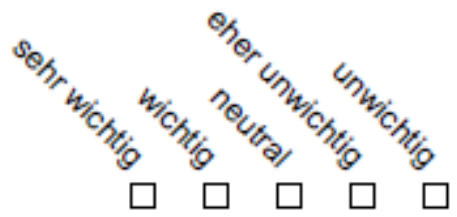

$\square \quad \square \quad \square \quad \square \quad \square$

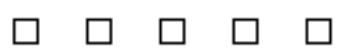

$\square \square \square \quad \square \quad \square$

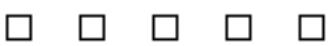

$\square \square \square \square \square$

$\square \quad \square \quad \square \quad \square \quad \square$

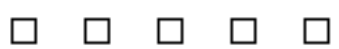

$\square \quad \square \quad \square \quad \square \quad \square$

$\square \quad \square \quad \square \quad \square \quad \square$

$\square \square \square \square \square$

$\square \quad \square \quad \square \quad \square \quad \square$

$\square \quad \square \quad \square \quad \square \quad \square$

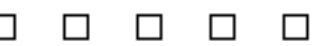

$\square \square \square \square \square$

$\square \quad \square \quad \square \quad \square \quad \square$

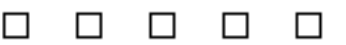

30. Gibt es erfolgsrelevante Eigenschaften, die Ihrer Meinung nach, stärker bei Ihren männlichen Kollegen zu beobachten sind?

31. Wie zufrieden sind Sie insgesamt mit

$\begin{array}{lll}\square 10 \% & \square 20 \% & \square 30 \% \\ \square 40 \% & \square 50 \% & \square 60 \% \\ \square 70 \% & \square 80 \% & \square 90 \%\end{array}$




\section{MUSTER}

EvaSys

[Copy]

Der folgende Fragenblock erfasst Ihre berufliche und allgemeine Zufriedenheit, sowie Ihre Selbsteinschätzung. [Fortsetzung]

32. Wie zufrieden sind Sie insgesamt mit Ihrer derzeitigen Lebenssituation?

$\begin{array}{lll}\square 10 \% & \square 20 \% & \square 30 \% \\ \square 40 \% & \square 50 \% & \square 60 \% \\ \square 70 \% & \square 80 \% & \square 90 \% \\ \square 100 \% & & \end{array}$

Vielen Dank für Ihre Teilnahme!

Bitte senden Sie mir die Ergebnisse der Umfrage an folgende E-Mail-Adresse:

Ihre Angaben werden nicht personenbezogen ausgewertet. Eine

Zuordnung der von Ihnen angegebenen E-Mail-Adresse zum ausgefüllten Fragebogen findet nicht statt. 


\subsection{Zusätzliche berufliche Qualifikationen der Teilnehmenden}

Aus Gründen der Übersicht wurden die Antworten in Rechtschreibung korrigiert und nach Subgruppen sortiert, Mehrfachantworten waren möglich, auf eine Trennung der Geschlechter in der Darstellung wurde aus Übersichtsgründen verzichtet;

$\mathrm{N}=148$, davon $\mathrm{n}=76$ weiblich, $\mathrm{n}=72$ männlich

Welche aktuelle Qualifikation nehmen Sie gegenwärtig ein?

Facharzt/ärztin für...

Chirurgische Forschung

Endokrine Chirurgie

Fachanatomie

$\mathrm{N}=1$

Immunologie

Med. Mikrobiologie/Virologie

Neurochirurgie

Phlebologie

Physikalische und rehabilitative Medizin

Proktologie

Rheumatologie, spezielle Rheumaorthopädie

(Kinder)Sportmedizin

Transplantationsmedizin

spezielle Unfallchirurgie

spezielle Viszeralchirurgie

$\mathrm{N}=3$

$\mathrm{N}=1$

$\mathrm{N}=1$

$\mathrm{N}=1$

$\mathrm{N}=1$

$\mathrm{N}=2$

$\mathrm{N}=6$

$\mathrm{N}=9$

$\mathrm{N}=8$

$\mathrm{N}=9$

$\mathrm{N}=4$

$\mathrm{N}=11$

$\mathrm{N}=4$

Zusätzliche Weiterbildungen/Zusatzbezeichnungen

\begin{tabular}{|l|l|}
\hline Bariatrische Chirurgie & $\mathrm{N}=1$ \\
\hline Balneotherapie & $\mathrm{N}=2$ \\
\hline Chirotherapie & $\mathrm{N}=4$ \\
\hline Ernährungsmedizin & $\mathrm{N}=1$ \\
\hline Gesundheitsökonom & $\mathrm{N}=1$ \\
\hline Handchirurgie & $\mathrm{N}=4$ \\
\hline Herzschrittmachertherapie & $\mathrm{N}=1$ \\
\hline Intensivmedizin & $\mathrm{N}=12$ \\
\hline Kinderorthopädie & $\mathrm{N}=4$ \\
\hline Medizinische Informatik & $\mathrm{N}=1$ \\
\hline
\end{tabular}




\begin{tabular}{|c|c|}
\hline Minimal-Intensive Chirurgie, LNA & $\mathrm{N}=1$ \\
\hline Naturheilverfahren & $\mathrm{N}=1$ \\
\hline Notfallmedizin & $\mathrm{N}=15$ \\
\hline Prüfärztin & $N=3$ \\
\hline Psychosomatik & $\mathrm{N}=1$ \\
\hline Qualitätsmanagement & $\mathrm{N}=5$ \\
\hline Radiologie & $\mathrm{N}=6$ \\
\hline Studienleiter & $\mathrm{N}=2$ \\
\hline Schmerztherapie & $\mathrm{N}=1$ \\
\hline Transfusionsbeauftragte & $\mathrm{N}=1$ \\
\hline Traumamanager & $\mathrm{N}=1$ \\
\hline Tropenmedizin & $\mathrm{N}=1$ \\
\hline Ultraschallanwendungen & $\mathrm{N}=1$ \\
\hline Wundmanager & $\mathrm{N}=1$ \\
\hline \multicolumn{2}{|l|}{ Bachelor /Master } \\
\hline Master of Medical Education & $N=3$ \\
\hline Diplom Gesundheitsökonom & $N=2$ \\
\hline Master of Health business administration (MHBA) & $N=5$ \\
\hline Master of Hospital Management & $\mathrm{N}=2$ \\
\hline $\mathrm{PhD}$ & $\mathrm{N}=1$ \\
\hline Technik in der Medizin & $\mathrm{N}=1$ \\
\hline Vorexamen Pharmazie & $\mathrm{N}=1$ \\
\hline
\end{tabular}




\subsection{Berufliche Tätigkeiten der Partnerinnen und Partner der} Teilnehmenden

\begin{tabular}{|c|c|}
\hline \multicolumn{2}{|c|}{ Welchen Beruf übt Ihr/e Partner/in aktuell aus? } \\
\hline Apothekerin & $N=1$ \\
\hline Architektin & $N=1$ \\
\hline Archäologin & $N=1$ \\
\hline Ärztin & $N=17$ \\
\hline Ärztin, aber derzeit in Elternzeit & $N=6$ \\
\hline Beamtin & $N=1$ \\
\hline Betriebswirtin & $N=1$ \\
\hline Designerin & $N=1$ \\
\hline Dipl. Pflegewirtin & $N=1$ \\
\hline freiberuflich & $N=1$ \\
\hline Fremdsprachensekretärin & $N=1$ \\
\hline Geschäftsführerin & $N=2$ \\
\hline Krankenpflege & $N=4$ \\
\hline Lehrerin & $N=3$ \\
\hline Managerin & $N=2$ \\
\hline Medizinproduktberaterin & $N=1$ \\
\hline Mutter & $N=1$ \\
\hline Pädagogin & $N=1$ \\
\hline Rechtsanwältin & $N=1$ \\
\hline Schauspielerin & $N=1$ \\
\hline Selbstständig & $N=1$ \\
\hline Studentin & $N=1$ \\
\hline Verwaltungsangestellte & $N=1$ \\
\hline
\end{tabular}




\begin{tabular}{|ll|}
\hline aktuell nicht berufstätig & $\mathrm{N}=2$ \\
\hline \hline Architekt & $\mathrm{N}=1$ \\
\hline Arzt & $\mathrm{N}=15$ \\
\hline Angestellter im DAX-Unternehmen & $\mathrm{N}=1$ \\
\hline Bank & $\mathrm{N}=1$ \\
\hline Forschung & $\mathrm{N}=2$ \\
\hline Geologe & $\mathrm{N}=2$ \\
\hline Geschäftsführer & $\mathrm{N}=2$ \\
\hline Ingenieur & $\mathrm{N}=2$ \\
\hline Journalist & $\mathrm{N}=1$ \\
\hline Jurist & $\mathrm{N}=3$ \\
\hline Personalberater & $\mathrm{N}=1$ \\
\hline Rettungsassistent & $\mathrm{N}=1$ \\
\hline Steuerberater & $\mathrm{N}=1$ \\
\hline Systemadministrator & $\mathrm{N}=1$ \\
\hline Transplantationskoordinator & $\mathrm{N}=1$ \\
\hline Universitätsangestellter & $\mathrm{N}=2$ \\
\hline promovierter Unternehmensberater & $\mathrm{N}=1$ \\
\hline Verwaltungs-Beamter & $\mathrm{N}=1$ \\
\hline Werbung & $\mathrm{N}=1$ \\
\hline & \\
\hline (a) & \\
\hline
\end{tabular}




\section{$7 \quad$ Literaturverzeichnis}

\subsection{Literatur}

Abele AE, Nitzsche U (2002): Der Schereneffekt bei der beruflichen Entwicklung von Ärztinnen und Ärzten. Dtsch Med Wochenschr 127, 2057-2062

Abele-Brehm A (2002): Nur am Anfang sind alle gleich... Forsch - Mitteilungen DFG 27, 4-6

Ackerly DC, Sangvai DG, Udayakumar K, Shah BR, Kalman NS, Cho AH, Schulman KA, Fulkerson WJ, Dzau VJ (2011): Training the Next Generation of PhysicianExecutives: An Innovative Residency Pathway in Management and Leadership. Acad Med $\underline{86}, 575-579$

Albrecht D (2010): Nachwuchsmangel in der Chirurgie-Was tun? Dtsch Ges Chir 39, 33-34

Asch DA, Jedrziewski MK, Christakis NA (1997): Response rates to mail surveys published in medical journals. J Clin Epidemiol 무, 1129-1136

Atesok KI, Hurwitz SR, Egol KA, Ahn J, Owens BD, Crosby LA, Pellegrini VD (2012): Perspective: Integrating Research Into Surgical Residency Education. Acad Med $\underline{87}$, $592-597$

Axinn WG, Link CF, Groves RM (2011): Responsive Survey Design, Demographic Data Collection, and Models of Demographic Behavior. Demography $\underline{48}, 1127-1149$

Azizzadeh A, McCollum CH, Miller III CC, Holliday KM, Shilstone HC, Lucci Jr A (2003): Factors influencing career choice among medical students interested in surgery. Curr Surg $\underline{60}, 210-213$

Baschera D, O'Donnell Taylor E, Masilonyane-Jones T, Isenegger P, Zellweger R (2015): Are Medical Students Who Want to Become Surgeons Different? An International Cross-Sectional Study. World J Surg 39, 2908-2918

Baxter N, Cohen R, McLeod R (1996): The impact of gender on the choice of surgery as a career. Am J Surg 172, 373-376

Bishawi M, Pryor AD (2014): Should technical aptitude evaluation become part of resident selection for surgical residency? Surg Endosc 28, 2761-2762

Bohrer T, Koller M, Schlitt HJ, Bauer H (2011): Workload and quality of life of surgeons. Results and implications of a large-scale survey by the German Society of Surgery. Langenbecks Arch Surg 396, 669-676

Buckley CE, Kavanagh DO, Gallagher TK, Conroy RM, Traynor OJ, Neary PC (2013): Does aptitude influence the rate at which proficiency is achieved for laparoscopic appendectomy? J Am Coll Surg 217, 1020-1027 
Buckley CE, Kavanagh DO, Nugent E, Ryan D, Traynor OJ, Neary PC (2014): The impact of aptitude on the learning curve for laparoscopic suturing. Am J Surg 207, 263-270

Buddeberg-Fischer B (2001): Karriereentwicklung von Frauen und Männern in der Medizin. Schweiz Ärzteztg $\underline{\text { 82, }}$ 1838-1844

Buddeberg-Fischer B, Stamm M (2010): The medical profession and young physicians' lifestyles in flux: challenges for specialty training and health care delivery systems. Swiss Med Wkly $\underline{140}$, w:13134

Buddeberg-Fischer B, Stamm M, Buddeberg C, Bauer G, Hämmig O, Klaghofer R (2008): Arbeitsstress, Gesundheit und Lebenszufriedenheit junger Ärztinnen und Ärzte. Dtsch Med Wochenschr 133, 2441-2447

Burgess DJ, Joseph A, van Ryn M, Carnes M (2012): Does Stereotype Threat Affect Women in Academic Medicine? Acad Med 87, 506-512

van den Bussche H (2012): Wie bitte geht's nach oben? - Karriereverläufe von Ärztinnen und Ärzten in der fachärztlichen Weiterbildung - Übersicht über eine multizentrische prospektive Studie. XX- Z Für Frauen Med 1, 136-141

Buxel H (2009): Motivation, Arbeitsplatzerwartungen/-zufriedenheit und Jobwahlverhalten von Assistenzärztinnen und -ärzten sowie Studierenden der Humanmedizin: Ergebnisse zweier empirischer Untersuchungen und Implikationen für das Personalmanagement und -marketing von Krankenhäusern. Vorlesung an der Fachhochschule Münster, Münster im April 2009. Dokument vorliegend.

Buxel H (2012): Arbeitsplatzbedingungen und -zufriedenheit von Ärztinnen und Ärzten in Krankenhäusern: Ergebnisse einer empirischen Untersuchung. Vorlesung an der Fachhochschule Münster, Münster im Juni 2012. Dokument vorliegend.

Carnes M, Morrissey C, Geller SE (2008): Women's Health and Women's Leadership in Academic Medicine: Hitting the Same Glass Ceiling? J Womens Health 17, 14531462

Carty SE, Colson YL, Garvey LS, Schuchert VD, Schwentker A, Tzeng E, Corcoran NA, Simmons RL, Webster MW, Billiar TR (2002): Maternity policy and practice during surgery residency: How we do it. Surgery 132, 682-688

Chen MM, Yeo HL, Roman SA, Bell RH, Sosa JA (2013): Life events during surgical residency have different effects on women and men over time. Surgery 154, 162-170

Cobey JC (2010): The Surgeon Shortage: Constructive Participation during Health Reform. J Am Coll Surg 211, 568

Cochran A, Hauschild T, Elder WB, Neumayer LA, Brasel KJ, Crandall ML (2013): Perceived gender-based barriers to careers in academic surgery. Am J Surg 206, 263268

Colletti L, Mulholland M, Sonnad S (2000): Perceived obstacles to career success for women in academic surgery. Arch Surg 135, 972-977 
Conrad P, Carr P, Knight S, Renfrew MR, Dunn MB, Pololi L (2010): Hierarchy as a Barrier to Advancement for Women in Academic Medicine. J Womens Health $15409996 \underline{19}$, 799-805

Corry NH, Williams CS, Battaglia M, McMaster HS, Stander VA (2017): Assessing and adjusting for non-response in the Millennium Cohort Family Study. BMC Med Res Methodol 17, 16

Cropsey KL, Masho SW, Shiang R, Sikka V, Kornstein SG, Hampton CL (2008): Why do faculty leave? Reasons for attrition of women and minority faculty from a medical school: four-year results. J Womens Health 2002 17, 1111-1118

Diener MK, Menger MD, Jähne J, Saeger H-D, Klar E (2014): Future perspectives for surgical research in Germany. Langenbecks Arch Surg 399, 253-262

Drosdeck JM, Osayi SN, Peterson LA, Yu L, Ellison EC, Muscarella P (2015): Surgeon and nonsurgeon personalities at different career points. J Surg Res $\underline{196}, 60-66$

Dyrbye LN, Shanafelt TD, Balch CM, Satele D, Sloan J, Freischlag J (2011): Relationship between work-home conflicts and burnout among American surgeons: a comparison by sex. Arch Surg 146, 211-217

Flannery AM (2002): Success, women, and academic surgery. Surgery 131, 670-671

Frank E, Brownstein M, Ephgrave K, Neumayer L (1998): Characteristics of women surgeons in the United States. Am J Surg 176, 244-250

Frank E, McMurray J, Linzer M, Elon L (1999): Career satisfaction of us women physicians: Results from the women physicians' health study. Arch Intern Med 159, 1417-1426

Gargiulo D, Hyman N, Herbert J (2006): Women in surgery: Do we really understand the deterrents? Arch Surg 141, 405-408

Gibis B, Müller C-H, Heinz A, Jacob R (2013): Bundesweite Befragung von Medizinstudierenden zu ihren Berufserwartungen. Dtsch Ärztebl 109, 327-332

Ginther DN, Dattani S, Miller S, Hayes P (2016): Thoughts of Quitting General Surgery Residency: Factors in Canada. J Surg Educ 73, 513-517

Gittes GK (2006): The surgeon-scientist in a new biomedical research era. Surgery $\underline{140,}, 123-$ 131

Goldberg LR (1990): An alternative „description of personality“: The Big-Five factor structure. J Pers Soc Psychol 모, 1216-1229

Gottesman MM (2013): The Role of the NIH in Nurturing Clinician-Scientists. N Engl J Med $\underline{368}, 2249-2251$

Grözinger M, Nesseler T, Schneider F (2010): Nachwuchskampagne Teil 12: Karriere oder

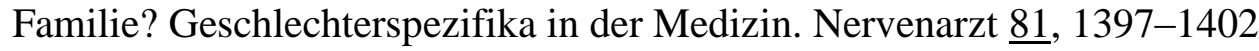


Hill E, Vaughan S (2013): The only girl in the room: how paradigmatic trajectories deter female students from surgical careers. Med Educ 47, 547-556

Hill EJR, Bowman KA, Stalmeijer RE, Solomon Y, Dornan T (2014): Can I cut it? Medical students' perceptions of surgeons and surgical careers. Am J Surg 208, 860-867

Hitchcock MA, Bland CJ, Hekelman FP, Blumenthal MG (1995): Professional networks: the influence of colleagues on the academic success of faculty. Acad Med J Assoc Am Med Coll $\underline{70}, 1108-1116$

Hoffman BM, Coons MJ, Kuo PC (2010): Personality differences between surgery residents, nonsurgery residents, and medical students. Surgery $\underline{148}, 187-193$

Hoover EL (2006): Mentoring women in academic surgery: overcoming institutional barriers to success. J Natl Med Assoc 98, 1542-1545

Jagsi R, Guancial EA, Worobey CC, Henault LE, Chang Y, Starr R, Tarbell NJ, Hylek EM (2006): The "Gender Gap" in Authorship of Academic Medical Literature - A 35Year Perspective. N Engl J Med $\underline{355}$, 281-287

Janus K, Amelung VE, Gaitanides M, Schwartz FW (2007): German physicians "on strike"Shedding light on the roots of physician dissatisfaction. Health Policy $\underline{82}$, 357-365

Kaderli R, Muff B, Stefenelli U, Businger A (2011): Female surgeons' mentoring experiences and success in an academic career in Switzerland. Swiss Med Wkly 141, w:13233

Kaplan SH, Sullivan LM, Dukes KA, Phillips CF, Kelch RP, Schaller JG (1996): Sex Differences in Academic Advancement - Results of a National Study of Pediatricians. N Engl J Med $\underline{335}, 1282-1290$

Kass RB, Souba WW, Thorndyke LE (2006): Challenges Confronting Female Surgical Leaders: Overcoming the Barriers. J Surg Res $\underline{132}$, 179-187

Kellerman S (2001): Physician response to surveys A review of the literature. Am J Prev Med $\underline{20}, 61-67$

Kerr H-L, Armstrong LA, Cade JE (2016): Barriers to becoming a female surgeon and the influence of female surgical role models. Postgrad Med J 92, 576-580

Klein J, Frie KG, Blum K, Siegrist J, von dem Knesebeck O (2010): Berufliche Gratifikationskrisen, Job Strain und Burnout bei chirurgisch tätigen Krankenhausärzten. Psychother Psych Med 60, 374-379

Krüger M (2009): Nachwuchsmangel in der Chirurgie. Unfallchirurg 112, 923-928

Kwong A, Chau WW, Kawase K (2014): Work-life balance of female versus male surgeons in Hong Kong based on findings of a questionnaire designed by a Japanese surgeon. Surg Today $\underline{44}, 62-72$

Lawler A (2006): Universities Urged to Improve Hiring and Advancement of Women. Science $\underline{313}, 1712-1712$ 
Levine RB, Lin F, Kern DE, Wright SM, Carrese J (2011): Stories From Early-Career Women Physicians Who Have Left Academic Medicine: A Qualitative Study at a Single Institution: Acad Med $\underline{86}, 752-758$

Levinson W, Tolle SW, Lewis C (1989): Women in academic medicine. Combining career and family. N Engl J Med $\underline{321}, 1511-1517$

Linn LS, Yager J, Cope D, Leake B (1985): Health status, job satisfaction, job stress, and life satisfaction among academic and clinical faculty. JAMA 254, 2775-2782

Lowenstein SR, Fernandez G, Crane LA (2007): Medical school faculty discontent: prevalence and predictors of intent to leave academic careers. BMC Med Educ $\underline{7}, 37$

Mache S, Vitzthum K, Klapp BF, Groneberg DA (2012): Improving quality of medical treatment and care: are surgeons' working conditions and job satisfaction associated to patient satisfaction? Langenbecks Arch Surg 397, 973-982

Mache S, Bernburg M, Vitzthum K, Groneberg DA, Klapp BF, Danzer G (2015): Managing work-family conflict in the medical profession: working conditions and individual resources as related factors. BMJ Open $\underline{5}, 68-71$

Mayer K, Ho H, Goodnight J (2001): Childbearing and child care in surgery. Arch Surg 136, 649-655

Mayr R (2008): Ärztinnen in Salzburg, Endbericht der Studie zur Lebens- und Arbeitssituation. Salzburger Arzt 21, 13-18

McCord JH, McDonald R, Leverson G, Mahvi DM, Rikkers LF, Chen HC, Weber SM (2007): Motivation to pursue surgical subspecialty training: is there a gender difference? J Am Coll Surg 205, 698-703

McGreevy J, Wiebe D (2002): A preliminary measurement of the surgical personality. Am J Surg $\underline{184}, 121-125$

Menger MD, Laschke MW (2012): Chirurgische Forschung in Deutschland. Chir $\underline{83}$, 309-314

Menger MD, Schilling MK, Schäfers H-J, Pohlemann T, Laschke MW (2012): How to ensure the survival of the surgeon-scientist? The Homburg Program. Langenbecks Arch Surg $\underline{397}, 619-622$

Neumayer L, Kaiser S, Anderson K, Barney L, Curet M, Jacobs D, Lynch T, Gazak C (2002): Perceptions of women medical students and their influence on career choice. Am J Surg $\underline{183}, 146-150$

Nonnemaker L (2000): Women Physicians in Academic Medicine - New Insights from Cohort Studies. N Engl J Med 342, 399-405

Parment A: Die Generation Y-Mitarbeiter der Zukunft. 1. Auflage; Gabler Verlag, Wiesbaden 2011 
Pololi LH, Civian JT, Brennan RT, Dottolo AL, Krupat E (2013): Experiencing the Culture of Academic Medicine: Gender Matters, A National Study. J Gen Intern Med 28, 201207

Ramirez AJ, Graham J, Richards MA, Gregory WM, Cull A (1996): Mental health of hospital consultants: the effects of stress and satisfaction at work. Lancet $\underline{347}, 724-728$

Rapp-Engels R, Gedrose B, Kaduszkiewicz H, Wonneberger C, van den Bussche H (2012): Memorandum zur Verbesserung der beruflichen Entwicklung von Ärztinnen. Ergebnisse der Arbeitstagung „Geschlechterspezifische Perspektiven und Umsetzungsstrategien in der fachärztlichen Weiterbildung“ (Hamburg 17.18.02.2012). Nachdr Z Allg Med 요, 320-321

Rasch D, Guiard V (2002): The robustness of parametric statistical methods. Psychol Sci $\underline{46}$, 175-208

Rasch D, Kubinger KD, Moder K (2009): The two-sample t test: pre-testing its assumptions does not pay off. Stat Pap 52, 219-231

Richards JMJ, Drummond R, Murray J, Fraser S, Macdonald A, Parks RW (2009): What proportion of basic surgical trainees continue in a surgical career? A survey of the factors which are important in influencing career decisions. Surgeon $\underline{7}, 270-275$

Richardson H, Redfern N (2000): Why do women reject surgical careers? Ann R Coll Surg Engl $\underline{\text { 82, }}$ 290-3

Richter-Kuhlmann E (2015): Mutterschutz: Operieren in der Schwangerschaft. Dtsch Ärztebl $\underline{112}, 123$

Riska E (2011): Gender and medical careers. Maturitas $\underline{68}, 264-267$

Römer F, Ziegler S, Scherer M, van den Bussche H (2017): Die Berufsverlaufszufriedenheit von Assistenzärzten und -ärztinnen nach vierjähriger Weiterbildung. ZEFQ 120, 4753

Rosta J, Gerber A (2008): Arbeitszufriedenheit bei Krankenhausärzten und -ärztinnen in Deutschland. Ergebnisse einer bundesweiten Erhebung im Herbst 2006. Gesundheitswesen $\underline{70}, 519-524$

Sanfey H, Saalwachter-Schulmann A, Nyhof-Young J, Eidelson B, Mann B (2006): Influences on medical student career choice: Gender or generation? Arch Surg 141, 1086-1094

Saunders CM, Nichevich A, Elllis C (2008): Frontiers in Academic Surgery: The Five M's. ANZ J Surg $\underline{78}, 350-355$

Scheuerlein H, Settmacher U (2010): Gedanken zur Aus- und Weiterbildung zum Chirurgen gestern, heute und morgen. ZBL Chir 135, 451-457

Schmidt CE, Möller J, Schmidt K, Gerbershagen MU, Wappler F, Limmroth V, Padosch SA, Bauer M (2011): Generation Y. Anaesthesist $\underline{60}, 517$ 
Schmidt K, Meyer J, Liebeneiner J, Schmidt CE, Hüttenbrink KB (2012): Generation Y in ENT: leading a young generation of doctors. HNO $\underline{60}, 993-1002$

Schroen AT, Brownstein MR, Sheldon GF (2004): Women in academic general surgery. Acad Med 79, 310-318

Schwartz RW, Barclay JR, Harrell PL, Murphy AE, Jarecky RK, Donnelly MB (1994): Defining the surgical personality: a preliminary study. Surgery $\underline{115}, 62-68$

Seemann NM, Webster F, Holden HA, Moulton CE, Baxter N, Desjardins C, Cil T (2016): Women in academic surgery: why is the playing field still not level? Am J Surg 211, 343-349

Sexton KW, Hocking KM, Wise E, Osgood MJ, Cheung-Flynn J, Komalavilas P, Campbell KE, Dattilo JB, Brophy CM (2012): Women in Academic Surgery: The Pipeline Is Busted. J Surg Educ $\underline{69}$, 84-90

Sheldon GF (2010): The Surgeon Shortage: Constructive Participation during Health Reform. J Am Coll Surg 210, 887-894

Siemiatycki J, Campbell S (1984): Nonresponse bias and early versus all responders in mail and telephone surveys. Am J Epidemiol 120, 291-301

Sonnad SS, Colletti LM (2002): Issues in the recruitment and success of women in academic surgery. Surgery $\underline{132}, 415-419$

Stabile B (2008): The surgeon: A changing profile. Arch Surg 143, 827-831

Steele CM (1997): A threat in the air: How stereotypes shape intellectual identity and performance. Am Psychol 52, 613-629

Stroh C (2010): Motivation von Frauen in der Chirurgie - Lust oder Last. Zentralb Chir $\underline{135}$, $447-450$

Sutton PA, Mason J, Vimalachandran D, McNally S (2014): Attitudes, motivators, and barriers to a career in surgery: a national study of U.K. undergraduate medical students. J Surg Educ 71, 662-667

Tardiff K, Cella D, Seiferth C, Perry S (1986): Selection and change of specialties by medical school graduates. J Med Educ $\underline{61}, 790-796$

Tesch BJ, Helen M, Amy L, Nattinger AB (1995): Promotion of Women Physicians in Academic Medicine: Glass Ceiling or Sticky Floor? JAMA 273, 1022-1025

Thomas JH (1997): The surgical personality: fact or fiction. Am J Surg 174, 573-577

Troppmann KM, Palis BE, Goodnight JE, Ho HS, Troppmann C (2009): Women surgeons in the new millennium. Arch Surg 144, 635-642

Wai PY, Dandar V, Radosevich DM, Brubaker L, Kuo PC (2014): Engagement, Workplace Satisfaction, and Retention of Surgical Specialists in Academic Medicine in the United States. J Am Coll Surg 219, 31-42 
Wallace JE, Lemaire JB, Ghali WA (2009): Physician wellness: a missing quality indicator. Lancet $\underline{374}, 1714-1721$

Weber T, Reidel M, Graf S, Hinz U, Keller M, Büchler MW (2005): Karriere im Gegenwind. Chirurg $\underline{76}, 703-711$

Weise K (2010): Zum Nachwuchsmangel in Unfallchirurgie und Orthopädie. Z Orthop Unf $\underline{148}, 17-18$

Wells SA (1996): The surgical scientist. Ann Surg 224, 239-254

Wendel TM, Godellas CV, Prinz RA (2003): Are there gender differences in choosing a surgical career? Surgery 134, 591-596

Wicker S, Rabenau HF, Haberl AE, Bühren A, Bechstein WO, Sarrazin CM (2011): Blutübertragbare Infektionen und die schwangere Mitarbeiterin im Gesundheitswesen. Chirurg $\underline{83}, 136-142$

Willett LL, Wellons MF, Hartig JR, Roenigk L, Panda M, Dearinger AT, Allison J, Houston TK (2010): Do Women Residents Delay Childbearing Due to Perceived Career Threats? Acad Med $\underline{85}, 640-646$

Williams C, Cantillon P (2000): A surgical career? The views of junior women doctors. Med Educ 34, 602-607

Williams Jr. TE, Ellison EC (2008): Population analysis predicts a future critical shortage of general surgeons. Surgery $\underline{144}, 548-556$

Wyrzykowski AD, Han E, Pettitt BJ, Styblo TM, Rozycki GS (2006): A Profile of Female Academic Surgeons: Training, Credentials, and Academic Success. Am Surg $\underline{72}$, $1153-1159$

Yedidia MJ, Bickel J (2001): Why aren't there more women leaders in academic medicine? the views of clinical department chairs. Acad Med $\underline{76}, 453-465$

Zerhouni EA (2005): Translational and Clinical Science — Time for a New Vision. N Engl J Med $\underline{353}, 1621-1623$

Zhuge Y, Kaufman J, Simeone DM, Chen H, Velazquez OC (2011): Is There Still a Glass Ceiling for Women in Academic Surgery? Ann Surg 253, 637-643

Ziegler S, van den Bussche H, Römer F, Krause-Solberg L, Scherer M (2017): Berufliche Präferenzen bezüglich Versorgungssektor und Position von Ärztinnen und Ärzten nach vierjähriger fachärztlicher Weiterbildung. Dtsch Med Wochenschr 142, e74-e82

\subsection{Internetquellen}

Berufsverband deutscher Chirurgen e.V. - Nachwuchsmangel und Nachwuchsförderung in der

Chirurgie. 
http://www.bdc.de/index_level3.jsp?documentid=0A309312613CE959C12577440036 BEC0\&form=Dokumente; Zugriff am 04.05.2014

Bundesärztekammer

Weiterbildungsordnung

Chirurgie. http://www.bundesaerztekammer.de/aerzte/aus-weiter-

fortbildung/weiterbildung/muster-weiterbildungsordnung/; Zugriff am 03.10.2016

Bundesärztekammer und Kassenärztliche Bundesvereinigung - Dem deutschen Gesundheitswesen gehen die Ärzte aus! Studie zur Altersstruktur und Arztzahlentwicklung. http://www.kbv.de/media/sp/Arztzahlstudie_2010.pdf; Zugriff am 29.08.2017

Bundesministerium für Familie, Senioren, Frauen und Jugend - Mehr Frauen profitieren künftig vom gesetzlichen Mutterschutz. https://www.bmfsfj.de/bmfsfj/aktuelles/allemeldungen/mehr-frauen-profitieren-kuenftig-vom-gesetzlichen-mutterschutz/109116; Zugriff am 14.12.2017

Chirurgie.uni-luebeck.de - $\quad$ FamSurg-Programm. http://www.chirurgie.uniluebeck.de/Forschung+und+Lehre/FamSurg_Programm-p-188.html; Zugriff am 11.08.2016

Ewi-psy.fu-berlin.de - Fragebogen und Interviewleitfaden im Projekt „PROFIL“ (Professionalisierung und Integration der Lebenssphären. Geschlechtsspezifische Berufsverläufe in Medizin und Psychologie) - Forschungsbericht Nr. 14. http://www.ewi-psy.fu-

berlin.de/einrichtungen/arbeitsbereiche/arbpsych/media/publikationen/forschungsberic hte/fb_14.pdf; Zugriff am 04.02.2018

Ewi-psy.fu-berlin.de - Unterschiedliche Berufsverläufe, Berufserfolg und Lebensbedingungen von Frauen und Männern in zwei hochqualifizierten Berufen - Forschungsbericht Nr. 16. http://www.ewi-psy.fuberlin.de/einrichtungen/arbeitsbereiche/arbpsych/media/publikationen/forschungsberic hte/fb_16.pdf; Zugriff am 04.02.2018

Marburger Bund - Für ein familienfreundliches Krankenhaus. https://www.marburgerbund.de/projekte/familienfreundliches-krankenhaus; Zugriff am 02.05.2014

Medizinische Fakultät Universität Wien - Frauenwege - Mut sichtbar machen. www.meduniwien.ac.at/typo3/uploads/media/8_10_07_Wissenbilanz.pdf; Zugriff am 14.03.2016

Med.uni-Goettingen.de - Habilitationsordnung der Georg August Universität Göttingen. www.med.uni-goettingen.de; Zugriff am 09.12.2015

Statistisches Bundesamt - Fächergruppe Humanmedizin/Gesundheitswissenschaften, Lehrund Forschungsbereich: Klinisch Praktische Humanmedizin (ohne Zahnmedizin) Wissenschaftliches und künstlerisches Personal - Fachgebiet Chirurgie. www.destatis.de; Zugriff am 28.07.2017

Statistisches Bundesamt - Pressemitteilungen - Frauenanteil bei den Habilitierten steigt weiterhin

kontinuierlich

an. 
https://www.destatis.de/DE/PresseService/Presse/Pressemitteilungen/2017/06/PD17_2 17_213.html; Zugriff am 25.07.2017

Statistisches Bundesamt - Prüfungen an Hochschulen - Fachserie 11 Reihe 4.2. https://www.destatis.de/DE/Publikationen/Thematisch/BildungForschungKultur/Hoch schulen/PruefungenHochschulen.html; Zugriff am 04.12.2017

Statistisches Bundesamt - Studierende an Hochschulen - Fachserie 11 Reihe 4.1 Wintersemester.

https://www.destatis.de/DE/Publikationen/Thematisch/BildungForschungKultur/Hoch schulen/StudierendeHochschulenEndg2110410177004.pdf?_blob=publicationFile; Zugriff am 04.12.2017

Statistisches Bundesamt- Grunddaten der Krankenhäuser- Fachserie 12 Reihe 6.1.1. https://www.destatis.de/DE/Publikationen/Thematisch/Gesundheit/Krankenhaeuser/Gr unddatenKrankenhaeuser.html; Zugriff am 04.12.2017

Thieme.de - PJ-Umfrage. https://www.thieme.de/viamedici/pj-pj-umfrage-1556.htm/a/pjumfrage-2014-21649.htm; Zugriff am 27.01.2016

Uke.de - Institut und Poliklinik für Allgemeinmedizin - Karriereverläufe von Ärztinnen und Ärzten in der fachärztlichen Weiterbildung (KarMed). http://www.uke.de/klinikeninstitute/institute/allgemeinmedizin/forschung/karriereverläufe-von-ärztinnen-undärzten-während-der-fachärztlichen-weiterbildung.html; Zugriff am 21.07.2017

Uke.de - Institut und Poliklinik für Allgemeinmedizin - Projekt Transfergendermed. https://www.uke.de/klinikeninstitute/institute/allgemeinmedizin/forschung/transferma\%C3\%9Fnahmen-zurgendergerechten-karrieref\% $3 \%$ B6rderung-von-frauen-in-der-medizin(transfergendermed)-folgeprojekt-von-karmed.html; Zugriff am 31.08.2017

Uni-luebeck.de - Leitfaden: Schwanger in der Chirurgie. http://www.opids.de/infomaterial/detailansicht-publikationen/artikel/leitfadenschwanger-in-der-chirurgie/; Zugriff am 10.08.2016 


\section{Danksagung:}

Ich danke Frau Prof. Dr. Sarah König für die hervorragende, zuverlässige, konstruktive und motivierende Unterstützung und Betreuung sowie Frau Sonia Sippel für ihre zuverlässige Hilfestellung und regelmäßigen Ratschläge in methodischen und statistischen Fragen. Außerdem Frau Prof. Dr. Hell für die initiale Ideenfindung. 